\title{
Electromagnetic two-body currents of one- and two-pion range
}

\author{
S. Pastore ${ }^{\mathrm{a}}$, R. Schiavilla ${ }^{\mathrm{a}, \mathrm{b}}$, and J.L. Goity ${ }^{\mathrm{b}, \mathrm{c}}$ \\ ${ }^{a}$ Department of Physics, Old Dominion University, Norfolk, VA 23529, USA \\ ${ }^{\mathrm{b}}$ Jefferson Lab, Newport News, VA 23606, USA \\ ${ }^{\mathrm{c}}$ Department of Physics, Hampton University, Hampton, VA 23668, USA
}

(Dated: December 2, 2018)

\begin{abstract}
Nuclear electromagnetic currents are derived in time-ordered perturbation theory within an effective-field-theory framework including explicit nucleons, $\Delta$ isobars, and pions up to one loop, or $\mathrm{N}^{3} \mathrm{LO}$. The currents obtained at $\mathrm{N}^{2} \mathrm{LO}$, i.e. ignoring loop corrections, are used in a study of neutron radiative captures on protons and deuterons at thermal energies, and of $A=2$ and 3 nuclei magnetic moments. The wave functions for $A=2$ are derived from solutions of the Schrödinger equation with the Argonne $v_{18}(\mathrm{AV} 18)$ or CD-Bonn (CDB) potentials, while those for $A=3$ are obtained with the hyperspherical-harmonics-expansion method from a realistic Hamiltonian including, in addition to the AV18 or CDB two-nucleon, also a three-nucleon potential. With the strengths of the $\Delta$-excitation currents occurring at $\mathrm{N}^{2} \mathrm{LO}$ determined to reproduce the $n-p$ cross section and isovector combination of the trinucleon magnetic moments, we find that the cross section and photon circular polarization parameter, measured in $n$ - $d$ and $\vec{n}-d$ processes, are underpredicted by theory, for example the cross section by (11-38)\% as the cutoff is increased from 500 to $800 \mathrm{MeV}$. A complete analysis of the results, in particular their large cutoff dependence, is presented.
\end{abstract}

PACS numbers: 12.39.Fe, 13.40.-f, 25.10.+s, 25.40.Lw 


\section{INTRODUCTION}

The present work is the first stage of a research program aimed at studying electromagnetic observables of light nuclei $(A \leq 8)$, and particularly radiative capture processes in the three- and four-nucleon systems, within a theoretical approach in which many-body electromagnetic current operators derived in chiral effective field theory $(\chi \mathrm{EFT})$ [1, 2, 3] are used in transition matrix elements between nuclear wave functions obtained from realistic Hamiltonians with two- and three-body potentials. This "hybrid" approach was adopted in a recent calculation of the astrophysical factor for the $p-p$ and $p-{ }^{3} \mathrm{He}$ fusion reactions by weak capture at the $\mathrm{keV}$ energies relevant in the interior of the Sun [4].

Neutron and proton radiative captures on ${ }^{2} \mathrm{H},{ }^{3} \mathrm{H}$ and ${ }^{3} \mathrm{He}$ are particularly challenging from the standpoint of nuclear few-body theory. This can be appreciated by comparing the measured values for the cross sections of thermal neutron radiative capture on ${ }^{1} \mathrm{H}$, ${ }^{2} \mathrm{H},{ }^{3} \mathrm{He}$. Their respective values in $\mathrm{mb}$ are: $(332.6 \pm 0.7)$ [5], (0.508 \pm 0.015$)$ [6], and $(0.055 \pm 0.003)$ [7]. Thus, in going from $A=2$ to 4 the cross section has dropped by almost four orders of magnitude. These processes are induced by magnetic dipole transitions between the initial two-cluster state in relative $\mathrm{S}$-wave and the final bound state. The ${ }^{3} \mathrm{H}$ and ${ }^{4} \mathrm{He}$ wave functions, respectively $\Psi_{3}$ and $\Psi_{4}$, are approximately eigenfunctions of the magnetic dipole operator $\boldsymbol{\mu}$, namely $\mu_{z} \Psi_{3} \simeq \mu_{p} \Psi_{3}$ and $\mu_{z} \Psi_{4} \simeq 0$, where $\mu_{p}=2.793 \mathrm{n} . \mathrm{m}$. is the proton magnetic moment - the experimental value of the ${ }^{3} \mathrm{H}$ magnetic moment is 2.979 n.m, while ${ }^{4} \mathrm{He}$ has no magnetic moment. These relations would be exact, if the ${ }^{3} \mathrm{H}$ and ${ }^{4} \mathrm{He}$ wave functions were to consist of the symmetric S-wave term only. In fact, tensor components in the nuclear potentials generate significant D-state admixtures, that partially spoil this eigenstate property. To the extent that it is approximately satisfied, though, the matrix elements $\left\langle\Psi_{3}\left|\mu_{z}\right| \Psi_{1+2}\right\rangle$ and $\left\langle\Psi_{4}\left|\mu_{z}\right| \Psi_{1+3}\right\rangle$ vanish due to orthogonality between the initial and final states. This orthogonality argument fails in the case of the deuteron, since then $\mu_{z} \Psi_{2} \simeq\left(\mu_{p}-\mu_{n}\right) \phi_{2}(S) \chi_{0}^{0} \eta_{0}^{1}$, where $\chi_{M_{S}}^{S}$ and $\eta_{M_{T}}^{T}$ are two-nucleon spin and isospin states, respectively. The magnetic dipole operator can therefore connect the large S-wave component $\phi_{2}(\mathrm{~S})$ of the deuteron to a $T=1{ }^{1} \mathrm{~S}_{0} n$ - $p$ state - the orthogonality between the latter and the deuteron follows from the orthogonality between their respective spin-isospin states.

As a result of this suppression, the $n-d, p-d, n-{ }^{3} \mathrm{He}$, and $p-{ }^{3} \mathrm{H}$ radiative (as well as $p-{ }^{3} \mathrm{He}$ weak) captures are very sensitive to small components in the wave functions, particularly the D-state admixtures generated by tensor forces, and to many-body terms in the electromagnetic (and weak) current operators.

There have been in the past several calculations of these processes in the conventional framework - referred to as the standard nuclear physics approach (SNPA) in Ref. [4] - see [8] and references therein. Some of these studies, in particular the recent ones of Ref. [9], have used accurate (essentially exact) bound and continuum wave functions corresponding to realistic Hamiltonians, which provide an excellent description of $A=3$ and 4 binding energies and radii, as well as of a variety of low-energy scattering observables (see [10] and references therein). The electromagnetic current operator includes, in addition to the standard convection and spin-magnetization terms of individual protons and neutrons, also two- and three-body terms, constructed from, respectively, the two- and three-nucleon potentials so as to satisfy exactly current conservation (CC) with them. The method by which this is achieved has been improved over the years [11], and its latest implementation is discussed at length in Ref. [9] - for an alternative formulation, though, see Ref. [12]. It is not unique, since 
obviously the CC relation puts no constraints on the transverse component of the current. Nevertheless, it does generate two- and three-body terms, whose behavior, particularly at short range, is consistent with that of the corresponding potentials. This behavior in the latter is ultimately "determined" by reproducing a set of experimental two- and three-nucleon scattering data and binding energies.

These currents have been shown to provide a very satisfactory description of a variety of electronuclear properties, including, for example, $n-p$ capture [9] and deuteron photodisintegration at low energy [13], and magnetic moments of $A=3-7$ nuclei [14]. Yet, they lead to $\sim 10 \%$ overestimate of the experimental cross section in $n$ - $d$ capture [9]. The discrepancy between theory and experiment increases to $\sim 60 \%$ in the case of the $n^{-}{ }^{3} \mathrm{He}$ capture cross section [15], although this earlier study, in contrast to that of Ref. [9], is not based on wave functions derived from the latest generation of potentials. The one-body (impulseapproximation or IA) term of the magnetic dipole operator gives, respectively, only $45 \%$ and $10 \%$ of the $n$ - $d$ and $n{ }^{3} \mathrm{He}$ cross-section experimental values because of the suppression mentioned above.

Electromagnetic currents up to one loop corrections have been derived in $\chi$ EFT within the heavy-baryon formalism by Park et al. in Ref. [16]. These currents were used in hybrid calculations of the $n-p$ capture cross section [16, 17], spin observables in $\vec{n}-\vec{p}$ capture [18], and, more recently, magnetic moments of the deuteron and trinucleons [17].

In the present work, we derive the electromagnetic currents by including, in addition to nucleon and pion, also $\Delta$-isobar degrees of freedom. Thus we assume that the $\Delta$-nucleon mass difference, just as the pion mass, is of the same order as the low momentum scale generically indicated by $Q$. Formal expressions up to one loop are obtained in time-ordered perturbation theory by employing non-relativistic Hamiltonians derived from the chiral Lagrangian formulation of Refs. [1, 2, 3]. The present study is similar to that of Ref. [16], albeit it uses a different formalism. Various aspects of the calculations are discussed in considerable detail. However, a discussion of renormalization is not given here: it is deferred to a later publication [19]. It is nonetheless opportune to comment on it. There are two stages of regularization necessary in the one loop calculations: the first is the usual regularization of the one-loop corrections to the potential and to the currents, and the second is the regularization necessary for solving the Schrödinger equation and for the calculation of the current matrix elements. All this must be followed by corresponding renormalization procedures. In the calculations to follow at next-to-next-to-leading order $\left(\mathrm{N}^{2} \mathrm{LO}\right)$, the only loop corrections needed are those of the one-body current, which can be absorbed into the proton and neutron magnetic moments and electromagnetic radii. Therefore the required regularization is in the calculation of the matrix elements of the two-body components of the current. The latter is implemented as usual through a short-range cutoff parameter and, although a full fledged renormalization is not carried out at this point, we expect that a choice of the cutoff equal to that in the input potentials will give realistic values for the matrix elements.

These $\mathrm{N}^{2} \mathrm{LO}$ currents are used to calculate the magnetic moments of $A=2$ and 3 nuclei, and thermal neutron radiative captures on protons and deuterons. Realistic two- and threenucleon (for $A=3$ ) potentials are used to generate the bound and continuum wave functions. To have an estimate of the model dependence arising from short-range phenomena, the variation of the predictions is studied as function of the short-range cutoff parameter mentioned above, which is used to regularize the two-body operators, as well as of the input potentials - either the Argonne $v_{18}$ (AV18) [20] or CD-Bonn (CDB) 21] in combination with 
respectively the Urbana IX [22] or Urbana IX* [23] — used to generate the wave functions (the AV18 and CDB have rather different short-range behaviors).

We find that the $\mathrm{N}^{2} \mathrm{LO}$ calculations do not provide a satisfactory description of the experimental data, particularly for the suppressed process ${ }^{2} \mathrm{H}(n, \gamma)^{3} \mathrm{H}$. This clearly points to the need of including loop corrections. However, it remains an interesting question whether these corrections will resolve the present discrepancies between theory and experiment.

This paper is organized into eight sections and four appendices. In Sec. III, we list, after defining our notation and conventions, the relevant strong- and electromagnetic-interaction Hamiltonians, obtained from chiral Lagrangians with nucleons, $\Delta$ isobars, and pions, while in Sec. III we derive the nuclear electromagnetic current up to $\mathrm{N}^{2} \mathrm{LO}$, i.e. ignoring loop corrections, in momentum space, and give the configuration-space representation of its operators in Sec. IV. Section V consists of two subsections: the first contains a derivation of one-loop two-body currents, while the second lists the two-body currents at $\mathrm{N}^{3} \mathrm{LO}$, implied by four-nucleon contact Lagrangians involving two gradients. In Sec. VI we show that the currents up to $\mathrm{N}^{3} \mathrm{LO}$ are conserved when used in combination with $\chi \mathrm{EFT}$ potential including corrections up to one loop. In Sec. VII, we present and discuss results for the magnetic moments of the deuteron and trinucleons, and for the radiative captures of thermal neutrons on protons and deuterons. Finally, in Sec. VIII we summarize our conclusions and outline the next stage in the research program initiated here. A number of details are relegated in the Appendices, including: expressions for the vertices associated with the interaction Hamiltonians of interest (Appendix $\mathrm{A}$ ); a collection of formulae relevant for the configuration-space representation of the $\mathrm{N}^{2} \mathrm{LO}$ operators (Appendix $\mathrm{B}$ ); a listing of the analytical expressions for the one-loop currents involving $\Delta$-isobars in the intermediate states (Appendix $\mathbb{C}$ ); and, lastly, a listing of the four-nucleon contact Hamiltonians (Appendix D).

\section{PRELIMINARIES}

Before listing the interaction Hamiltonians, it is useful to define our notation and conventions. In the Schrödinger picture adopted in the present study, the isospin triplet of pion fields $\pi_{a}(\mathbf{x})$ and their canonical conjugates $\Pi_{a}(\mathbf{x})$, with $a=x, y, z$, are represented as

$$
\begin{aligned}
& \pi_{a}(\mathbf{x})=\sum_{\mathbf{p}} \frac{1}{\sqrt{2 \omega_{p}}}\left[c_{\mathbf{p}, a} \mathrm{e}^{i \mathbf{p} \cdot \mathbf{x}}+\text { h.c. }\right], \\
& \Pi_{a}(\mathbf{x})=\sum_{\mathbf{p}} i \sqrt{\frac{\omega_{p}}{2}}\left[c_{\mathbf{p}, a} \mathrm{e}^{i \mathbf{p} \cdot \mathbf{x}}-\text { h.c. }\right],
\end{aligned}
$$

where the annihilation and creation operators $c_{\mathbf{p}, a}$ and $c_{\mathbf{p}, a}^{\dagger}$ satisfy standard commutation relations, and $\omega_{p} \equiv\left(p^{2}+m_{\pi}^{2}\right)^{1 / 2}$. The nucleon and $\Delta$-isobar fields, respectively $N(\mathbf{x})$ and $\Delta(\mathbf{x})$, with their corresponding canonical conjugates $i N^{\dagger}(\mathbf{x})$ and $i \Delta^{\dagger}(\mathbf{x})$, are given, in the non-relativistic limit of interest here, as

$$
\begin{aligned}
& N(\mathbf{x})=\sum_{\mathbf{p}, \sigma \tau} b_{\mathbf{p}, \sigma \tau} \mathrm{e}^{i \mathbf{p} \cdot \mathbf{x}} \chi_{\sigma \tau}, \\
& \Delta(\mathbf{x})=\sum_{\mathbf{p}, \sigma_{\Delta} \tau_{\Delta}} d_{\mathbf{p}, \sigma_{\Delta} \tau_{\Delta}} \mathrm{e}^{i \mathbf{p} \cdot \mathbf{x}} \chi_{\sigma_{\Delta} \tau_{\Delta}},
\end{aligned}
$$

where the $b$ 's and $d$ 's are annihilation operators for nucleons and $\Delta$ isobars, and $\chi_{\sigma \tau}$ and $\chi_{\sigma_{\Delta} \tau_{\Delta}}$ denote their respective spin-isospin states. Again, the $b$ 's and $b^{\dagger}$ 's, and similarly the $d$ 's and $d^{\dagger}$ 's, satisfy the standard anticommutation relations, appropriate for fermionic fields. 
Standard time-ordered perturbation theory is used to calculate the transition amplitude:

$$
\left\langle N^{\prime} N^{\prime}|T| N N ; \gamma\right\rangle=\left\langle N^{\prime} N^{\prime}\left|H_{1} \sum_{n=1}^{\infty}\left(\frac{1}{E_{i}-H_{0}+i \eta} H_{1}\right)^{n-1}\right| N N ; \gamma\right\rangle,
$$

where $|N N ; \gamma\rangle\left(\left|N^{\prime} N^{\prime}\right\rangle\right)$ represents, in a schematic notation, an initial (final) state containing two nucleons and a photon (two nucleons only) of energy $E_{i}\left(E_{f}=E_{i}\right), H_{0}$ is the Hamiltonian describing free pions, nucleons and $\Delta$-isobars, and $H_{1}$ is the Hamiltonian describing interactions among these particles as well as their couplings to the electromagnetic field. The evaluation of this amplitude is carried out in practice by inserting complete sets of eigenstates of $H_{0}$ between successive terms of $H_{1}$ in the expansion above. However, since we are only interested in retaining irreducible contributions, we omit from these intermediate states those that contain only two nucleons (this aspect of the present calculations is elaborated on in Secs. IIIA and $\mathrm{VA}$ ). Thus, the nuclear electromagnetic current $\mathbf{j}$ of interest here is obtained from

$$
\left.\left\langle N^{\prime} N^{\prime}|T| N N ; \gamma\right\rangle\right|_{\text {irreducible }}=-\frac{\hat{\mathbf{e}}_{\mathbf{q} \lambda}}{{\sqrt{2 \omega_{q}}}_{q}} \cdot \mathbf{j},
$$

where $\mathbf{q}, \omega_{q}$, and $\hat{\mathbf{e}}_{\mathbf{q} \lambda}$ denote respectively the photon momentum, energy, and polarization, and only first-order contributions in the electromagnetic interaction are retained in the evaluation of the transition amplitude.

\section{A. Pion, nucleon, and $\Delta$-isobar interaction Hamiltonians}

The interaction Hamiltonians involving nucleon, $\Delta$-isobar, and pion fields are derived from the effective chiral Lagrangian approach formulated in Refs. [1, 2]. Their explicit expressions are given by

$$
\begin{aligned}
H_{\pi N N} & =\frac{g_{A}}{F_{\pi}} \int \mathrm{d} \mathbf{x} N^{\dagger}(\mathbf{x})\left[\boldsymbol{\sigma} \cdot \nabla \pi_{a}(\mathbf{x})\right] \tau_{a} N(\mathbf{x}), \\
H_{\pi N \Delta} & =\frac{h_{A}}{F_{\pi}} \int \mathrm{d} \mathbf{x} \Delta^{\dagger}(\mathbf{x})\left[\mathbf{S} \cdot \nabla \pi_{a}(\mathbf{x})\right] T_{a} N(\mathbf{x})+\text { h.c. }, \\
H_{\pi \pi N N} & =\frac{1}{F_{\pi}^{2}} \int \mathrm{d} \mathbf{x} N^{\dagger}(\mathbf{x})[\boldsymbol{\pi}(\mathbf{x}) \times \mathbf{\Pi}(\mathbf{x})] \cdot \boldsymbol{\tau} N(\mathbf{x}),
\end{aligned}
$$

where $g_{A} \simeq 1.25$ and $F_{\pi} \simeq 186 \mathrm{MeV}$ are the nucleon axial coupling constant and pion decay amplitude, respectively, $h_{A}$ is the $\pi N \Delta$ coupling constant, and $S_{\alpha}$ and $T_{a}$ are transition spin and isospin operators, converting a nucleon into a $\Delta$ isobar and satisfying

$$
S_{\alpha}^{\dagger} S_{\beta}=\frac{2}{3} \delta_{\alpha \beta}-\frac{i}{3} \epsilon_{\alpha \beta \gamma} \sigma_{\gamma},
$$

and similarly for $T_{a}^{\dagger} T_{b}$ (note that in Ref. [2] the isospin transition operator is half that defined here).

In addition to these, there is a set of four-fermion contact interactions described by

$$
H_{\mathrm{CT}, 1}=\sum_{\alpha=S, T} \frac{C_{\alpha}}{2} \int \mathrm{d} \mathbf{x}\left[N^{\dagger}(\mathbf{x}) \boldsymbol{\Gamma}_{\alpha} N(\mathbf{x})\right] \cdot\left[N^{\dagger}(\mathbf{x}) \boldsymbol{\Gamma}_{\alpha} N(\mathbf{x})\right]
$$




$$
\begin{aligned}
& H_{\mathrm{CT}, 2}=D_{T} \int \mathrm{d} \mathbf{x}\left[N^{\dagger}(\mathbf{x}) \boldsymbol{\sigma} \tau_{a} N(\mathbf{x})\right] \cdot\left[\Delta^{\dagger}(\mathbf{x}) \mathbf{S} T_{a} N(\mathbf{x})\right]+\text { h.c. } \\
& H_{\mathrm{CT}, 3}=\sum_{\alpha=S, T} C_{\alpha}^{\prime} \int \mathrm{d} \mathbf{x}\left[N^{\dagger}(\mathbf{x}) \boldsymbol{\Gamma}_{\alpha} N(\mathbf{x})\right] \cdot\left[\Delta^{\dagger}(\mathbf{x}) \boldsymbol{\Gamma}_{\alpha}^{\prime} \Delta(\mathbf{x})\right], \\
& H_{\mathrm{CT}, 4}=D_{T}^{\prime} \int \mathrm{d} \mathbf{x}\left[\Delta^{\dagger}(\mathbf{x}) \mathbf{S} T_{a} N(\mathbf{x})\right] \cdot\left[\Delta^{\dagger}(\mathbf{x}) \mathbf{S} T_{a} N(\mathbf{x})\right]+\text { h.c. }, \\
& H_{\mathrm{CT}, 5}=D_{T}^{\prime \prime} \int \mathrm{d} \mathbf{x}\left[\Delta^{\dagger}(\mathbf{x}) \mathbf{S} T_{a} N(\mathbf{x})\right] \cdot\left[N^{\dagger}(\mathbf{x}) \mathbf{S}^{\dagger} T_{a}^{\dagger} \Delta(\mathbf{x})\right],
\end{aligned}
$$

where we have defined

$$
\boldsymbol{\Gamma}_{S}=\boldsymbol{\Gamma}_{S}^{\prime}=1, \quad \boldsymbol{\Gamma}_{T}=\boldsymbol{\sigma}, \quad \boldsymbol{\Gamma}_{T}^{\prime}=\boldsymbol{\Sigma},
$$

and $\boldsymbol{\Sigma} / 2$ is the spin-3/2 operator. As it will become clear below, terms involving more than two $\Delta$-isobars are not needed in the present study. Finally, when discussing the renormalization of the two-body currents at tree level in a later work [19], we shall also need to consider the following Hamiltonians involving three- and four-pion interactions,

$$
\begin{gathered}
H_{3 \pi}=-\frac{g_{A}}{F_{\pi}^{3}} \int \mathrm{d} \mathbf{x} \boldsymbol{\pi}^{2}(\mathbf{x}) N^{\dagger}(\mathbf{x})\left[\boldsymbol{\sigma} \cdot \nabla \pi_{a}(\mathbf{x})\right] \tau_{a} N(\mathbf{x}), \\
H_{4 \pi}=\frac{1}{2 F_{\pi}^{2}} \int \mathrm{d} \mathbf{x}\left[\left[\boldsymbol{\pi}^{2}(\mathbf{x}) \boldsymbol{\Pi}^{2}(\mathbf{x})-\boldsymbol{\pi}^{2}(\mathbf{x}) \nabla \pi_{a}(\mathbf{x}) \cdot \nabla \pi_{a}(\mathbf{x})+\text { h.c. }\right]-m_{\pi}^{2}\left[\boldsymbol{\pi}^{2}(\mathbf{x})\right]^{2}\right],
\end{gathered}
$$

obtained by including corrections up to $\pi^{2}(\mathbf{x}) / F_{\pi}^{2}$ in the expansion of $D^{-1}$ factors, where $D \equiv 1+\boldsymbol{\pi}^{2}(\mathbf{x}) / F_{\pi}^{2}$, entering the chiral Lagrangians [1] .

\section{B. Electromagnetic interactions}

The charged pion field is defined as

$$
\pi_{\mp}(\mathbf{x})=\frac{1}{\sqrt{2}}\left[\pi_{x}(\mathbf{x}) \mp i \pi_{y}(\mathbf{x})\right],
$$

and minimal substitution,

$$
\nabla \pi_{\mp}(\mathbf{x}) \rightarrow[\nabla \mp i e \mathbf{A}(\mathbf{x})] \pi_{\mp}(\mathbf{x}),
$$

in the pion-derivative couplings leads to the interaction Hamiltonians:

$$
\begin{aligned}
& H_{\gamma \pi N N}=-e \frac{g_{A}}{F_{\pi}} \epsilon_{a b z} \int \mathrm{d} \mathbf{x} \mathbf{A}(\mathbf{x}) \cdot N^{\dagger}(\mathbf{x}) \boldsymbol{\sigma} \tau_{a} N(\mathbf{x}) \pi_{b}(\mathbf{x}) \\
& H_{\gamma \pi N \Delta}=-e \frac{h_{A}}{F_{\pi}} \epsilon_{a b z} \int \mathrm{d} \mathbf{x} \mathbf{A}(\mathbf{x}) \cdot \Delta^{\dagger}(\mathbf{x}) \mathbf{S} T_{a} N(\mathbf{x}) \pi_{b}(\mathbf{x})+\text { h.c. } \\
& H_{\gamma \pi \pi}=-e \epsilon_{a b z} \int \mathrm{d} \mathbf{x} \mathbf{A}(\mathbf{x}) \cdot\left[\nabla \pi_{a}(\mathbf{x})\right] \pi_{b}(\mathbf{x}) \\
& H_{\gamma \pi \pi N N}=-\frac{e}{2 m_{N}} \frac{1}{F_{\pi}^{2}} \int \mathrm{d} \mathbf{x} \mathbf{A}(\mathbf{x}) \cdot\left[N^{\dagger}(\mathbf{x})[i(\vec{\nabla}-\overleftarrow{\nabla})+\boldsymbol{\sigma} \times(\vec{\nabla}+\overleftarrow{\nabla})] \tau_{a} N(\mathbf{x})\right] \\
& \quad \times\left[\pi_{a}(\mathbf{x}) \pi_{z}(\mathbf{x})-\delta_{a, z} \pi^{2}(\mathbf{x})\right] \\
& H_{\gamma 3 \pi}=e \frac{g_{A}}{F_{\pi}^{3}} \epsilon_{a b z} \int \mathrm{d} \mathbf{x} \mathbf{A}(\mathbf{x}) \cdot N^{\dagger}(\mathbf{x}) \boldsymbol{\sigma} \tau_{a} N(\mathbf{x}) \pi_{b}(\mathbf{x}) \boldsymbol{\pi}^{2}(\mathbf{x}) \\
& H_{\gamma 4 \pi}=e \frac{2}{F_{\pi}^{2}} \epsilon_{a b z} \int \mathrm{d} \mathbf{x} \mathbf{A}(\mathbf{x}) \cdot\left[\nabla \pi_{a}(\mathbf{x})\right] \pi_{b}(\mathbf{x}) \boldsymbol{\pi}^{2}(\mathbf{x})
\end{aligned}
$$


where $e(>0)$ is the electric charge, and the transverse vector field $\mathbf{A}(\mathbf{x})$ (in Coulomb gauge) is expanded as

$$
\mathbf{A}(\mathbf{x})=\sum_{\mathbf{p}} \sum_{\lambda=1,2} \frac{1}{\sqrt{2 \omega_{p}}}\left[a_{\mathbf{p}, \lambda} \mathrm{e}^{i \mathbf{p} \cdot \mathbf{x}} \hat{\mathbf{e}}_{\mathbf{p}, \lambda}+\text { h.c. }\right] .
$$

The linear polarization (unit) vectors $\hat{\mathbf{e}}_{\mathbf{p}, 1}, \hat{\mathbf{e}}_{\mathbf{p}, 2}$ form along with $\hat{\mathbf{p}}$ a right-handed orthonormal system of axes, $\hat{\mathbf{e}}_{\mathbf{p}, 1} \times \hat{\mathbf{e}}_{\mathbf{p}, 2}=\hat{\mathbf{p}}$.

The interactions of individual nucleons and $\Delta$-isobars with the electromagnetic field are described by the following Hamiltonians:

$$
\begin{aligned}
H_{\gamma N N} & =\frac{e}{2 m_{N}} \int \mathrm{d} \mathbf{x} N^{\dagger}(\mathbf{x})\left[i e_{N}[-\overleftarrow{\nabla} \cdot \mathbf{A}(\mathbf{x})+\mathbf{A}(\mathbf{x}) \cdot \vec{\nabla}]\right. \\
& \left.-\mu_{N} \boldsymbol{\sigma} \cdot \nabla \times \mathbf{A}(\mathbf{x})\right] N(\mathbf{x}) \\
H_{\gamma N \Delta} & =-\frac{e \mu^{*}}{2 m_{N}} \int \mathrm{d} \mathbf{x} \Delta^{\dagger}(\mathbf{x}) \mathbf{S} \cdot[\nabla \times \mathbf{A}(\mathbf{x})] T_{z} N(\mathbf{x})+\text { h.c. }
\end{aligned}
$$

with

$$
e_{N}=\left(1+\tau_{z}\right) / 2, \quad \kappa_{N}=\left(\kappa_{S}+\kappa_{V} \tau_{z}\right) / 2, \quad \mu_{N}=e_{N}+\kappa_{N},
$$

where $\kappa_{S}$ and $\kappa_{V}$ are the isoscalar and isovector combinations of the anomalous magnetic moments of the proton and neutron $\left(\kappa_{S}=-0.12\right.$ n.m. and $\kappa_{V}=3.706$ n.m. $)$, and $\mu^{*}$ is the $N \Delta$-transition magnetic moment ( $\mu^{*} \simeq 3$ n.m.). In the $\gamma N \Delta$ term we only take into account the dominant magnetic dipole (M1) coupling, ignoring the much smaller Coulomb (C2) and electric quadrupole (E2) couplings. The expressions in Eqs. (2.28) and (2.29) result from considering the non-relativistic limit of the effective Hamiltonians with non-minimal couplings

$$
\begin{aligned}
H_{\gamma N N}^{\mathrm{R}} & =e \int \mathrm{d} \mathbf{x} \bar{\psi}_{N}(\mathbf{x})\left[e_{N} A^{\mu}(\mathbf{x}) \gamma_{\mu}+\frac{\kappa_{N}}{4 m_{N}} \sigma_{\mu \nu} F^{\mu \nu}(\mathbf{x})\right] \psi_{N}(\mathbf{x}) \\
H_{\gamma N \Delta}^{\mathrm{R}} & =-i \frac{e \mu^{*}}{2 m_{N}} \int \mathrm{d} \mathbf{x} \bar{\psi}_{\Delta}^{\mu}(\mathbf{x}) g_{\mu \lambda} \gamma_{\nu} \gamma_{5} T_{z} \psi_{N}(\mathbf{x}) F^{\nu \lambda}(\mathbf{x})+\text { h.c. }
\end{aligned}
$$

where $\psi_{N}(\mathbf{x})$ and $\psi_{\Delta}^{\mu}(\mathbf{x})$ are the spinor and spinor-vector fields describing the nucleon and $\Delta$ isobar, and $F^{\nu \lambda}(\mathbf{x})$ is the electromagnetic field tensor. The Bjorken and Drell conventions [24] are used for relativistic four-vectors, $\gamma$-matrices, and Dirac spin- $1 / 2$ spinors, except that the latter are taken to be normalized as $u^{\dagger}(\mathbf{p}, s) u(\mathbf{p}, s)=1$. The Rarita-Schwinger spin-3/2 spinors are defined as

$$
u^{\mu}\left(\mathbf{p}, s_{\Delta}\right)=\sum_{\lambda s}\left\langle 1 \lambda, 1 / 2 s \mid 3 / 2 s_{\Delta}\right\rangle \epsilon^{\mu}(\mathbf{p}, \lambda) u(\mathbf{p}, s)
$$

where in the particle rest-frame the four-vector $\epsilon^{\mu}$ is space-like, $\epsilon^{\mu}=\left(0, \hat{\boldsymbol{\epsilon}}_{\lambda}\right)$, and $\lambda= \pm 1,0$ denote spherical components.

\section{Power counting}

We denote generically by $Q$ a "small momentum", i.e. $Q \ll M$, where $M \simeq 1 \mathrm{GeV}$ is the typical hadronic mass scale, and consider as effective degrees of freedom the nucleon, 
$\Delta$-isobar, and pion. Thus, we assume that the pion mass and the mass difference between the $\Delta$-isobar and nucleon are both of order $Q, m_{\pi} \sim Q$ and $m_{\Delta}-m_{N} \sim Q$. However, the photon energy $\omega_{q}$ is assumed to be suppressed by an additional factor $Q / M$ relative to this small-momentum scale, i.e. $\omega_{q} \sim Q^{2} / M$. A generic coupling constant of dimensions (energy) ${ }^{\alpha}$ is assumed to scale with $M$ as $g=\tilde{g} M^{\alpha}$ with the expectation that $\widetilde{g} \simeq 1$.

Contributions to the transition amplitude in Eq. (2.6) can be organized as an expansion in powers of $Q / M[1]$. The power counting implied by the interaction Hamiltonians in the previous two sections can be easily inferred by examining the structure of their associated vertices, listed in Appendix A. This power counting is summarized in Table I.

\begin{tabular}{c|c||c|c}
\hline \hline & $Q$-scaling & & $Q$-scaling \\
\hline$H_{\pi N N}$ & $Q$ & $H_{\gamma \pi N N}$ & $e Q^{0}$ \\
$H_{\pi N \Delta}$ & $Q$ & $H_{\gamma \pi N \Delta}$ & $e Q^{0}$ \\
$H_{\pi \pi N N}$ & $Q$ & $H_{\gamma \pi \pi}$ & $e Q$ \\
$H_{\mathrm{CT}, 1-5}$ & $Q^{0}$ & $H_{\gamma \pi \pi N N}$ & $e Q$ \\
$H_{3 \pi}$ & $Q$ & $H_{\gamma 3 \pi}$ & $e Q^{0}$ \\
$H_{4 \pi}$ & $Q^{2}$ & $H_{\gamma 4 \pi}$ & $e Q$ \\
& & $H_{\gamma N N}$ & $e Q$ \\
& & $H_{\gamma N \Delta}$ & $e Q$ \\
\hline \hline
\end{tabular}

TABLE I: Powers of $Q$, the small momentum scale, associated with the vertices from the strongand electromagnetic-interaction Hamiltonians of Secs. IA IIB.

In the perturbative series, Eq. (2.5), a generic irreducible contribution will be characterized by a certain number, say $N$, of vertices, each scaling as $Q^{\alpha_{i}} \times Q^{-\beta_{i} / 2}(i=1, \ldots, N)$, where $\alpha_{i}$ is the power counting in Table \ and $\beta_{i}$ is the number of pions in and/or out of the vertex (this last factor is associated with the $1 / \sqrt{2 \omega_{p}}$ included in the pion field), a corresponding $N-1$ number of energy denominators, and possibly $L$ loops. Each of the energy denominators will involve pion energies and/or $\Delta N$ mass differences, both of order $Q$, as well as kinetic energies of nucleons and/or $\Delta$ isobars, which, however, are suppressed by a further $Q / M$ factor relative to $Q$. Loops on the other hand will contribute a factor $Q^{3}$ each, since they involve integrations over intermediate three momenta. Hence the power counting associated with such a contribution is

$$
\text { irreducible contribution }=\left(\prod_{i=1}^{N} Q^{\alpha_{i}-\beta_{i} / 2}\right) \times Q^{-(N-1)} \times Q^{3 L} .
$$

When one expands the nucleon propagators in powers of the kinetic energy around the static limit, terms with higher powers of $Q$ appear as the kinetic energy is order $Q^{2}$.

The power counting of Eq. (2.34) can also be obtained by considering the Feynman diagram where the loop integrals are carried out in four dimensions. The power of $Q$ of an irreducible diagram is then given by $4 L-2 n_{\pi}-n_{N}+\sum_{i=1}^{N} \alpha_{i}$, where $n_{\pi}$ is the number of pion propagators, and $n_{N}$ the number of nucleon propagators. This equation together with the topological relation $n_{\pi}+n_{N}=L+N-1$ leads to the power counting of Eq. (2.34).

Finally, the transition amplitude in Eq. (2.6) can be represented diagrammatically as in Fig. 1. The disconnected contributions in panels a) and b) will each contain a $\delta$-function in the initial and final three momenta of one of the two particles, for example panel a) $\propto \delta\left(\mathbf{p}_{2}^{\prime}-\mathbf{p}_{2}\right)$, and will therefore be enhanced by a factor $Q^{-3}$ relative to the connected (and 
irreducible) contributions in panel c). The power counting of diagrams a) and b) is then $e Q \times Q^{-3}=e Q^{-2}$. In fact, these diagrams are the leading contributions to the nuclear electromagnetic current. This fact certainly fits in well with what is known empirically about, for example, magnetic moments of nuclei or radiative captures, such as the ${ }^{1} \mathrm{H}(n, \gamma){ }^{2} \mathrm{H}$ process considered later in this work.

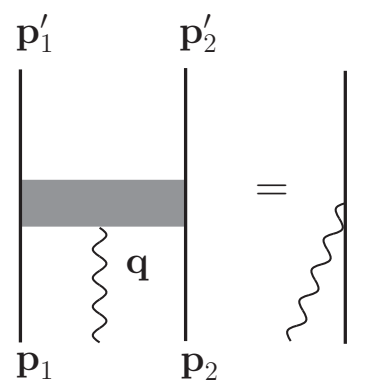

a)
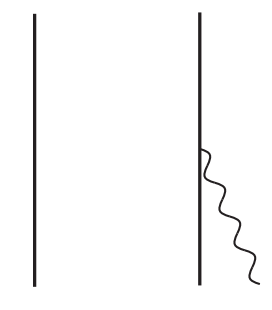

b)

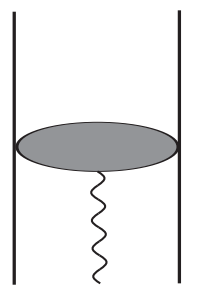

c)

FIG. 1: Schematic representation of the disconnected, a) and b), and connected, c), contributions to the $N N \gamma \rightarrow N N$ amplitude. Solid and wavy lines denote nucleons and photons, respectively.

\section{CURRENTS UP TO N $\mathrm{N}^{2} \mathrm{LO}$}

In this section we derive the nuclear electromagnetic currents up to next-to-next-toleading order $\left(\mathrm{N}^{2} \mathrm{LO}\right)$, that is $e Q^{0}$. The relevant contributions are illustrated in Figs. 2 and 3 , The expressions below are given in momentum space, configuration-space representations are discussed in Sec. IV]. The momenta are defined as

$$
\mathbf{k}_{i}=\mathbf{p}_{i}^{\prime}-\mathbf{p}_{i}, \quad \mathbf{K}_{i}=\left(\mathbf{p}_{i}^{\prime}+\mathbf{p}_{i}\right) / 2
$$

where $\mathbf{p}_{i}$ and $\mathbf{p}_{i}^{\prime}$ are the initial and final momenta of nucleon $i$. The leading order (LO), $e Q^{-2}$, is given by the one-body current, panel a) in Fig. 2 ,

$$
\mathbf{j}_{\mathrm{a}}^{\mathrm{LO}}=\frac{e}{2 m_{N}}\left[2 e_{N, 1} \mathbf{K}_{1}+i \mu_{N, 1} \boldsymbol{\sigma}_{1} \times \mathbf{q}\right]+1 \rightleftharpoons 2,
$$

where $\mathbf{q}$ is the photon momentum, $\mathbf{q}=\mathbf{k}_{i}$.

The contributions to next-to-leading order (NLO), $e Q^{-1}$, are represented by diagrams b)-c) and d)-i) in Fig. 2. A straightforward evaluation of these diagrams in the static limit leads to the expressions:

$$
\begin{aligned}
& \mathbf{j}_{\mathrm{b}-\mathrm{c}}^{\mathrm{NLO}}=-i e \frac{g_{A}^{2}}{F_{\pi}^{2}}\left(\boldsymbol{\tau}_{1} \times \boldsymbol{\tau}_{2}\right)_{z} \frac{1}{k_{2}^{2}+m_{\pi}^{2}} \boldsymbol{\sigma}_{1}\left(\boldsymbol{\sigma}_{2} \cdot \mathbf{k}_{2}\right)+1 \rightleftharpoons 2 \\
& \mathbf{j}_{\mathrm{d}-\mathrm{i}}^{\mathrm{NLO}}=i e \frac{g_{A}^{2}}{F_{\pi}^{2}}\left(\boldsymbol{\tau}_{1} \times \boldsymbol{\tau}_{2}\right)_{z} \frac{\mathbf{k}_{1}-\mathbf{k}_{2}}{\left(k_{1}^{2}+m_{\pi}^{2}\right)\left(k_{2}^{2}+m_{\pi}^{2}\right)}\left(\boldsymbol{\sigma}_{1} \cdot \mathbf{k}_{1}\right)\left(\boldsymbol{\sigma}_{2} \cdot \mathbf{k}_{2}\right)
\end{aligned}
$$

where the momenta transferred to nucleons 1 and 2 add up to $\mathbf{q}, \mathbf{k}_{1}+\mathbf{k}_{2}=\mathbf{q}$.

At next-to-next-to-leading order $\left(\mathrm{N}^{2} \mathrm{LO}\right), e Q^{0}$, there are two distinct contributions, illustrated in Fig. 3. The first is due to $(Q / M)^{2}$ corrections to the one-body current in Eq. (3.2). 


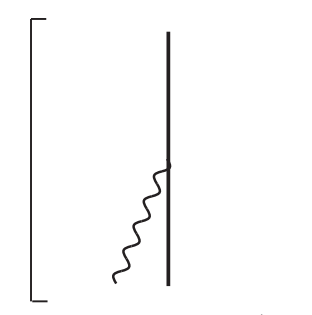

a)

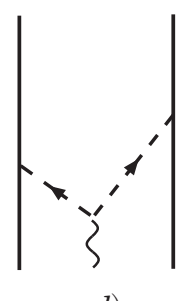

d)

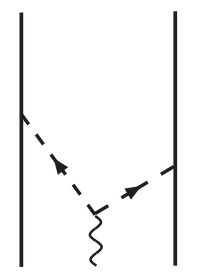

g)

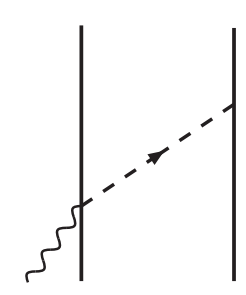

b)

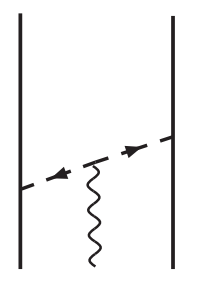

e)

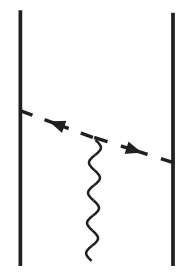

h)

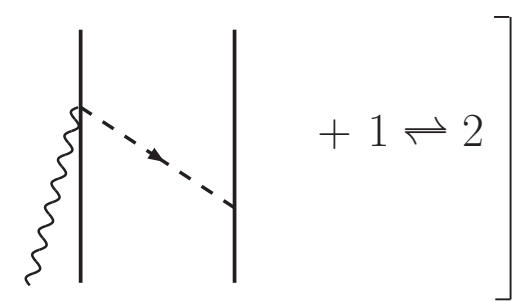

c)
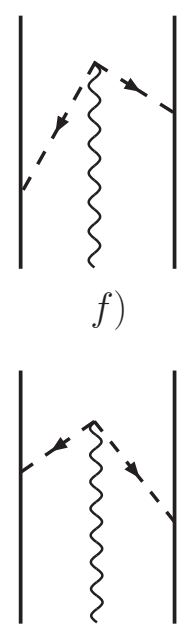

i)

FIG. 2: The complete set of time-ordered diagrams contributing at LO and NLO. Nucleons, pions, and photons are denoted by solid, dashed, and wavy lines, respectively.

These are easily derived from a non-relativistic expansion of Eq. (2.31):

$$
\begin{gathered}
\mathbf{j}_{\mathrm{a}}^{\mathrm{N}^{2} \mathrm{LO}=-} \frac{e}{8 m_{N}^{3}} e_{N, 1}\left[2\left(K_{1}^{2}+q^{2} / 4\right)\left(2 \mathbf{K}_{1}+i \boldsymbol{\sigma}_{1} \times \mathbf{q}\right)+\mathbf{K}_{1} \cdot \mathbf{q}\left(\mathbf{q}+2 i \boldsymbol{\sigma}_{1} \times \mathbf{K}_{1}\right)\right] \\
-\frac{i e}{8 m_{N}^{3}} \kappa_{N, 1}\left[\mathbf{K}_{1} \cdot \mathbf{q}\left(4 \boldsymbol{\sigma}_{1} \times \mathbf{K}_{1}-i \mathbf{q}\right)-\left(2 i \mathbf{K}_{1}-\boldsymbol{\sigma}_{1} \times \mathbf{q}\right) q^{2} / 2\right. \\
\left.+2\left(\mathbf{K}_{1} \times \mathbf{q}\right) \boldsymbol{\sigma}_{1} \cdot \mathbf{K}_{1}\right]+1 \rightleftharpoons 2 .
\end{gathered}
$$

At this point we should comment on the one-loop corrections to the one-body current, see Fig. 4. They occur at NLO and $\mathrm{N}^{2} \mathrm{LO}$, and are absorbed into the anomalous magnetic moments and electromagnetic radii of the proton and neutron.

The second class of $\mathrm{N}^{2} \mathrm{LO}$ contributions, represented by diagrams b)-i), involve $\Delta$-isobar excitation, and therefore vertices from $H_{\gamma N \Delta}, H_{\pi N \Delta}$, and $H_{\mathrm{CT}, 2}$. In the static limit, we find:

$$
\begin{aligned}
\mathbf{j}_{\mathrm{b}-\mathrm{g}}^{\mathrm{N}^{2} \mathrm{LO}} & =i \frac{e \mu^{*}}{9 m_{N}} \frac{g_{A} h_{A}}{\Delta F_{\pi}^{2}} \frac{\boldsymbol{\sigma}_{2} \cdot \mathbf{k}_{2}}{k_{2}^{2}+m_{\pi}^{2}}\left[4 \tau_{2, z} \mathbf{k}_{2}-\left(\boldsymbol{\tau}_{1} \times \boldsymbol{\tau}_{2}\right)_{z} \boldsymbol{\sigma}_{1} \times \mathbf{k}_{2}\right] \times \mathbf{q}+1 \rightleftharpoons 2, \\
\mathbf{j}_{\mathrm{h}-\mathrm{i}}^{\mathrm{N}^{2} \mathrm{LO}} & =-i \frac{e \mu^{*}}{9 m_{N}} \frac{D_{T}}{\Delta}\left[4 \tau_{2, z} \boldsymbol{\sigma}_{2}-\left(\boldsymbol{\tau}_{1} \times \boldsymbol{\tau}_{2}\right)_{z} \boldsymbol{\sigma}_{1} \times \boldsymbol{\sigma}_{2}\right] \times \mathbf{q}+1 \rightleftharpoons 2,
\end{aligned}
$$

where $\Delta$ is the $\Delta-N$ mass difference, $\Delta=m_{\Delta}-m_{N}$, and use has been made of the identities in Eq. (2.10) to eliminate spin- and isospin-transition operators in favor of Pauli spin and isospin matrices. 


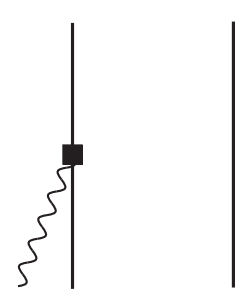

a)

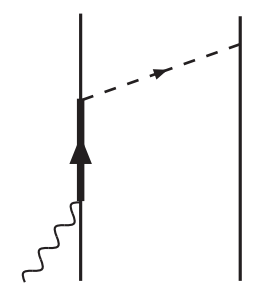

b)

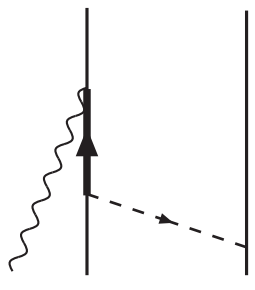

e)

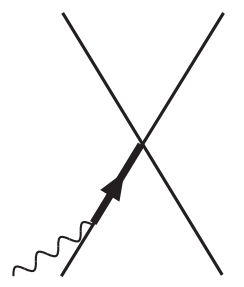

h)

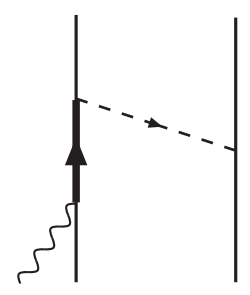

c)

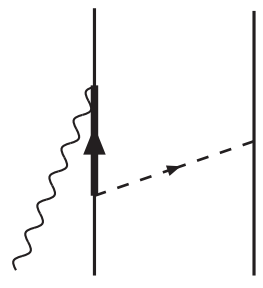

f)

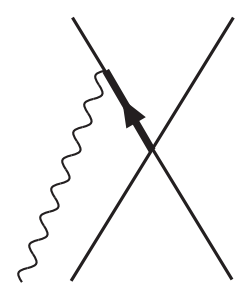

i)

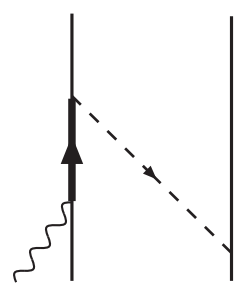

d)

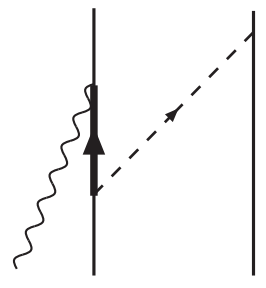

g)

FIG. 3: The complete set of time-ordered diagrams contributing at $\mathrm{N}^{2} \mathrm{LO}$. The square represents the $(Q / M)^{2}$ correction to the LO one-body current, while $\Delta$ isobars are denoted by thick solid lines, otherwise the notation is as in Fig. 2 ,

We conclude this section by noting that the expressions in Eqs. (3.3)-(3.4) and (3.6) are the well known pion seagull and in-flight, and $\Delta$-excitation currents commonly used in the literature (see, for example, the classic work of Ref. [25]).

\section{A. Recoil corrections: cancellations at $\mathrm{N}^{2} \mathbf{L O}$}

In the present formulation based on time-independent perturbation theory, there are in principle the additional $\mathrm{N}^{2} \mathrm{LO}$ contributions represented by diagrams a) and b) in Fig. 5 , However, these are exactly canceled by recoil corrections, also entering at $\mathrm{N}^{2} \mathrm{LO}$, to the reducible diagrams c)-f). For example, the contribution of diagrams $c$ ) $+d$ ) is given by

$$
\begin{gathered}
\mathrm{c}+\mathrm{d})=\frac{V_{\gamma N N}(1) V_{\pi N N}(1) V_{\pi N N}(2)}{E_{i}-E_{p}-E_{2}^{\prime}-\omega_{q}+i \eta}\left[\frac{1}{E_{i}-E_{p}-E_{2}-\omega_{q}-\omega_{k}+i \eta}\right. \\
\left.+\frac{1}{E_{i}-E_{1}-E_{2}^{\prime}-\omega_{q}-\omega_{k}+i \eta}\right]
\end{gathered}
$$

where the $V(i)$ 's denote the vertices from the interaction Hamiltonians relative to nucleon $i$, and the labeling of momenta is as illustrated in the figure. The initial and final energies $E_{i}$ and $E_{f}\left(E_{i}=E_{f}\right)$ are $E_{i}=E_{1}+E_{2}+\omega_{q}$ and $E_{f}=E_{1}^{\prime}+E_{2}^{\prime}$, while $E_{p}$ is the energy of the 
intermediate nucleon. These energies are all suppressed by $Q / M$ relative to $\omega_{k} \sim Q$, and therefore the denominators in square brackets can be expanded as

$$
[\cdots] \simeq-\frac{2}{\omega_{k}}-\frac{E_{i}-E_{p}-E_{2}^{\prime}-\omega_{q}}{\omega_{k}^{2}}
$$

so that the contribution of diagrams $\mathrm{c}+\mathrm{d}$ ) now reads

$$
\text { c) }+\mathrm{d})=V_{\gamma N N}(1) \frac{1}{E_{i}-E_{p}-E_{2}^{\prime}-\omega_{q}+i \eta} v^{\pi}(\mathbf{k})\left[1+\frac{E_{i}-E_{p}-E_{2}^{\prime}-\omega_{q}}{2 \omega_{k}}\right] \text {, }
$$

where $v^{\pi}(\mathbf{k})$ is the static one-pion-exchange potential (OPEP), defined as

$$
v^{\pi}(\mathbf{k}) \equiv-\frac{2}{\omega_{k}} V_{\pi N N}(1) V_{\pi N N}(2)=-\frac{g_{A}^{2}}{F_{\pi}^{2}} \frac{\boldsymbol{\sigma}_{1} \cdot \mathbf{k} \boldsymbol{\sigma}_{2} \cdot \mathbf{k}}{k^{2}+m_{\pi}^{2}} \boldsymbol{\tau}_{1} \cdot \boldsymbol{\tau}_{2} .
$$

The second term on the r.h.s. exactly cancels the contribution due to (the irreducible) diagram a) in Fig. 5, as can be easily surmised by noting that, in the static limit, the two energy denominators occurring in this diagram are each given by $\left(-1 / \omega_{k}\right)$, and therefore

$$
\text { a) }=-\frac{V_{\gamma N N}(1) v^{\pi}(\mathbf{k})}{2 \omega_{k}}
$$

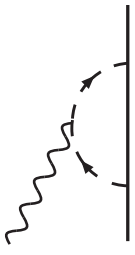

a)

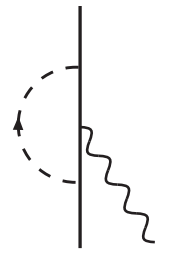

b)

FIG. 4: NLO and $\mathrm{N}^{2} \mathrm{LO}$ corrections to the one-body current. Notation is as in Fig. 2.

The discussion above becomes more delicate when the intermediate states describe fully interacting rather than free particles. Let $\delta v^{\pi}$ be the recoil correction to the static OPEP $v^{\pi}$, and let $|\varphi\rangle$ denote a bound or continuum state corresponding to $H_{0}+v^{\pi}$ with energy $E$. To first order in $\delta v^{\pi}$, the perturbed state $|\psi\rangle$ is

$$
|\psi\rangle=|\varphi\rangle+\frac{1}{E-H_{0}-v^{\pi}} \delta v^{\pi}|\varphi\rangle \equiv|\varphi\rangle+|\delta \varphi\rangle
$$

and the matrix element of the current operator $\mathbf{j}$ between initial and final states $\left|\psi_{i}\right\rangle$ and $\left|\psi_{f}\right\rangle$ can be expressed as

$$
\begin{aligned}
\left\langle\psi_{f}|\mathbf{j}| \psi_{i}\right\rangle & =\left\langle\varphi_{f}|\mathbf{j}| \varphi_{i}\right\rangle+\left\langle\varphi_{f}\left|\mathbf{j}^{\mathrm{LO}}\right| \delta \varphi_{i}\right\rangle+\left\langle\delta \varphi_{f}\left|\mathbf{j}^{\mathrm{LO}}\right| \varphi_{i}\right\rangle \\
& =\left\langle\varphi_{f}|\mathbf{j}| \varphi_{i}\right\rangle+\left\langle\varphi_{f}\right| \mathbf{j}^{\mathrm{LO}} \frac{1}{E_{i}-H_{0}-v^{\pi}} \delta v^{\pi}+\text { h.c. }\left|\varphi_{i}\right\rangle
\end{aligned}
$$



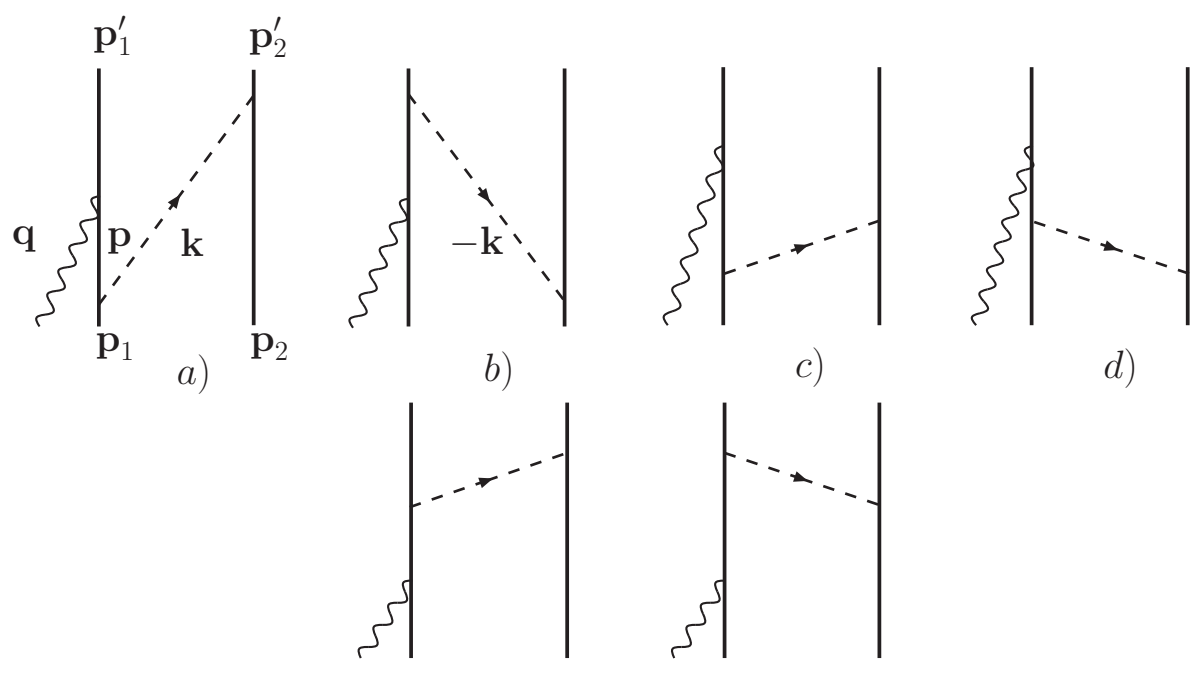

e)

$f)$

FIG. 5: Time-ordered diagrams illustrating the cancellation of the irreducible contributions a) and b) by the recoil corrections to the LO diagrams c)-f). Notation is as in Fig. 2 ,

where we have dropped terms of order $(\delta \varphi)^{2}$ as well as corrections beyond LO to the current in the matrix elements between $\left|\varphi_{i, f}\right\rangle$ and $\left|\delta \varphi_{f, i}\right\rangle$. In the analysis following Eq. (3.8), in which the nucleonic intermediate states are free particles, the recoil correction can be written as

$$
\left.\delta v^{\pi}\right|_{\text {free }}=\left(E_{i}-H_{0}\right) \frac{v^{\pi}}{2 \omega}+\text { h.c. , }
$$

where $\omega$ is the pion energy. In momentum space the expression for $\left.\delta v^{\pi}\right|_{\text {free }}$ coincides with that implied by Eq. (3.10), namely

$$
\left.\delta v^{\pi}\left(\mathbf{k}, \mathbf{K}_{1}, \mathbf{K}_{2}\right)\right|_{\text {free }}=\frac{v^{\pi}(\mathbf{k})}{2 \omega_{k}} \frac{\mathbf{k} \cdot\left(\mathbf{K}_{1}-\mathbf{K}_{2}\right)}{m_{N}},
$$

with $\mathbf{K}_{i}$ defined as in Eq. (3.1).

If we assume that the nucleonic intermediate states describe fully interacting particles, i.e. they are eigenstates of $H_{0}+v^{\pi}$, then it is plausible that the correction $\delta v^{\pi}$ should be expressed as

$$
\delta v^{\pi}=\left(E_{i}-H_{0}-v^{\pi}\right) \frac{v^{\pi}}{2 \omega}+\text { h.c. , }
$$

from which it follows that

$$
\left\langle\psi_{f}|\mathbf{j}| \psi_{i}\right\rangle=\left\langle\varphi_{f}\right| \mathbf{j}^{\mathrm{LO}}+\mathbf{j}^{\mathrm{NLO}}-\left(\frac{v^{\pi}}{2 \omega} \mathbf{j}^{\mathrm{LO}}+\text { h.c. }\right)\left|\varphi_{i}\right\rangle+\left\langle\varphi_{f}\right| \frac{v^{\pi}}{2 \omega} \mathbf{j}^{\mathrm{LO}}+\text { h.c. }\left|\varphi_{i}\right\rangle \text {. }
$$

The last two terms exactly cancel the two-body current contribution represented in Fig. 5 panels a)-b), namely the terms in brackets. Thus, if OPEP is taken in the static limit, Eq. (3.11), as is the case for the calculations reported below, then the contributions of diagrams a) and b) should not be retained, since they are canceled by recoil corrections to OPEP. 


\section{CURRENTS IN CONFIGURATION SPACE}

The calculations of electromagnetic observables reported in Sec. VII are carried out in configuration space, and hence configuration-space representations of the current operators are needed. Those of the one-body operators in Eqs. (3.2) and (3.5), generically denoted as $\mathbf{j}^{(1)}$, follow from

$$
\mathbf{j}^{(1)}(\mathbf{q})=\int_{\mathbf{k}_{1}} \int_{\mathbf{K}_{1}} \mathrm{e}^{i \mathbf{k}_{1} \cdot\left(\mathbf{r}_{1}^{\prime}+\mathbf{r}_{1}\right) / 2} \mathrm{e}^{i \mathbf{K}_{1} \cdot\left(\mathbf{r}_{1}^{\prime}-\mathbf{r}_{1}\right)} \bar{\delta}\left(\mathbf{k}_{1}-\mathbf{q}\right) \mathbf{j}^{(1)}\left(\mathbf{k}_{1}, \mathbf{K}_{1}\right),
$$

while those for the two-body current operators $\mathbf{j}^{(2)}$ are derived from

$$
\mathbf{j}^{(2)}(\mathbf{q})=\int_{\mathbf{k}_{1}} \int_{\mathbf{k}_{2}} \mathrm{e}^{i \mathbf{k}_{1} \cdot \mathbf{r}_{1}} \mathrm{e}^{i \mathbf{k}_{2} \cdot \mathbf{r}_{2}} \bar{\delta}\left(\mathbf{k}_{1}+\mathbf{k}_{2}-\mathbf{q}\right) \mathbf{j}^{(2)}\left(\mathbf{k}_{1}, \mathbf{k}_{2}\right)
$$

where the momenta $\mathbf{k}_{i}$ and $\mathbf{K}_{i}$ are defined as in Eq. (3.1), and we have introduced the notation

$$
\int_{\mathbf{p}} \equiv \int \frac{\mathrm{d} \mathbf{p}}{(2 \pi)^{3}}, \quad \bar{\delta}(\ldots) \equiv(2 \pi)^{3} \delta(\ldots)
$$

Note that $\mathbf{K}_{i} \rightarrow-i \nabla_{i}^{\prime} \delta\left(\mathbf{r}_{i}^{\prime}-\mathbf{r}_{i}\right)$, i.e. the configuration-space representation of the momentum operator. The LO current is then found to have the standard expression associated with the nucleon's convection and spin-magnetization currents,

$$
\mathbf{j}_{\mathrm{a}}^{\mathrm{LO}}(\mathbf{q})=\frac{e}{2 m_{N}}\left(e_{N, 1}\left[-i \nabla_{1}, \mathrm{e}^{i \mathbf{q} \cdot \mathbf{r}_{1}}\right]_{+}+i \mu_{N, 1} \boldsymbol{\sigma}_{1} \times \mathbf{q} \mathrm{e}^{i \mathbf{q} \cdot \mathbf{r}_{1}}\right)+1 \rightleftharpoons 2,
$$

where $[\ldots, \ldots]_{+}$denotes the anticommutator - this notation is also used in Eq. (4.11) below.

At NLO and $\mathrm{N}^{2} \mathrm{LO}$, however, the operators have $1 / r^{2}$ and $1 / r^{3}$ singularities $(r$ is the interparticle separation), which need to be regularized in order to avoid divergencies in the matrix elements of these operators between nuclear wave functions. We adopt a simple regularization procedure [2], i.e. a momentum-space cutoff. While its precise functional form is arbitrary, the choice made here of a Gaussian cutoff function,

$$
C_{\Lambda}(p)=\mathrm{e}^{-(p / \Lambda)^{2}}
$$

with the parameter $\Lambda \leq M \simeq 1 \mathrm{GeV}$, is merely dictated by convenience, since it leads to analytical expressions for the Fourier transforms below. It is expected that this arbitrariness be of little relevance, since the dependence of theoretical predictions on variations in the cutoff is (or should be) largely removed by a renormalization of the theory free parameters, which are fixed by reproducing a given set of observables. We shall return to this issue later, in Secs. VII and VIII.

The two-body currents at NLO are obtained as

$$
\begin{aligned}
& \mathrm{j}_{\mathrm{b}-\mathrm{c}}^{\mathrm{NLO}}(\mathbf{q})=\frac{e g_{A}^{2}}{F_{\pi}^{2}}\left(\boldsymbol{\tau}_{1} \times \boldsymbol{\tau}_{2}\right)_{z} \mathrm{e}^{i \mathbf{q} \cdot \mathbf{r}_{1}} \boldsymbol{\sigma}_{1}\left(\boldsymbol{\sigma}_{2} \cdot \nabla\right) f_{\Lambda}(r)+1 \rightleftharpoons 2 \\
& \mathbf{j}_{\mathrm{d}-\mathrm{i}}^{\mathrm{NLO}}(\mathbf{q})=\frac{e g_{A}^{2}}{F_{\pi}^{2}}\left(\boldsymbol{\tau}_{1} \times \boldsymbol{\tau}_{2}\right)_{z} \mathrm{e}^{i \mathbf{q} \cdot \mathbf{R}}\left[\boldsymbol{\sigma}_{1} \cdot(\nabla+i \mathbf{q} / 2)\right]\left[\boldsymbol{\sigma}_{2} \cdot(\nabla-i \mathbf{q} / 2)\right] \nabla g_{\Lambda}(\mathbf{r}, \mathbf{q})
\end{aligned}
$$


where $\mathbf{r}$ and $\mathbf{R}$ denote the relative and center-of-mass position vectors, respectively $\mathbf{r}=\mathbf{r}_{1}-\mathbf{r}_{2}$ and $\mathbf{R}=\left(\mathbf{r}_{1}+\mathbf{r}_{2}\right) / 2$, and the functions $f_{\Lambda}(r)$ and $g_{\Lambda}(\mathbf{r}, \mathbf{q})$ are defined as

$$
\begin{aligned}
f_{\Lambda}(r) & =\int_{\mathbf{p}} \mathrm{e}^{-i \mathbf{p} \cdot \mathbf{r}} \frac{C_{\Lambda}(p)}{p^{2}+m_{\pi}^{2}} \\
g_{\Lambda}(\mathbf{r}, \mathbf{q}) & =\int_{-1}^{+1} \mathrm{~d} x \mathrm{e}^{-i x \mathbf{q} \cdot \mathbf{r} / 2} \int_{\mathbf{p}} \mathrm{e}^{-i \mathbf{p} \cdot \mathbf{r}} \frac{C_{\Lambda}(p)}{\left[p^{2}+L^{2}(q ; x)\right]^{2}},
\end{aligned}
$$

with

$$
L(q ; x)=\sqrt{m_{\pi}^{2}+\left(1-x^{2}\right) q^{2} / 4} .
$$

Note that standard Feynman parametrization techniques have been employed to express $g_{\Lambda}$ in the form given above. We defer to Appendix $B$ for a listing of the formulae resulting from application of the gradients to $f_{\Lambda}(r)$ and $g_{\Lambda}(\mathbf{r}, \mathbf{q})$.

Finally, at $\mathrm{N}^{2} \mathrm{LO}$ the one-body term, Eq. (3.5), reads

$$
\begin{aligned}
\mathbf{j}_{\mathrm{a}}^{\mathrm{N}^{2} \mathrm{LO}}(\mathbf{q}) & =-\frac{e}{16 m_{N}^{3}} e_{N, 1}\left[-2 i\left(-\nabla_{1}^{2}+q^{2} / 4\right)\left(2 \nabla_{1}-\boldsymbol{\sigma}_{1} \times \mathbf{q}\right)\right. \\
& \left.-i \nabla_{1} \cdot \mathbf{q}\left(\mathbf{q}+2 \boldsymbol{\sigma}_{1} \times \nabla_{1}\right), \mathrm{e}^{i \mathbf{q} \cdot \mathbf{r}_{1}}\right]_{+}-\frac{i e}{16 m_{N}^{3}} \kappa_{N, 1}\left[-\nabla_{1} \cdot \mathbf{q}\left(4 \boldsymbol{\sigma}_{1} \times \nabla_{1}+\mathbf{q}\right)\right. \\
& \left.-\left(2 \nabla_{1}-\boldsymbol{\sigma}_{1} \times \mathbf{q}\right) q^{2} / 2-2\left(\nabla_{1} \times \mathbf{q}\right) \boldsymbol{\sigma}_{1} \cdot \nabla_{1}, \mathrm{e}^{i \mathbf{q} \cdot \mathbf{r}_{1}}\right]_{+}+1 \rightleftharpoons 2
\end{aligned}
$$

while the two-body terms are given by

$$
\begin{gathered}
\mathbf{j}_{\mathrm{b}-\mathrm{g}}^{\mathrm{N} 2 \mathrm{~L}}(\mathbf{q})=i \frac{e \mu^{*}}{9 m_{N}} \frac{g_{A} h_{A}}{\Delta F_{\pi}^{2}} \mathrm{e}^{i \mathbf{q} \cdot \mathbf{r}_{1}} \mathbf{q} \times\left[4 \tau_{2, z} \nabla-\left(\boldsymbol{\tau}_{1} \times \boldsymbol{\tau}_{2}\right)_{z} \boldsymbol{\sigma}_{1} \times \nabla\right] \boldsymbol{\sigma}_{2} \cdot \nabla f_{\Lambda}(r)+1 \rightleftharpoons 2, \\
\mathbf{j}_{\mathrm{h}-\mathrm{i}}^{\mathrm{N} 2}(\mathbf{q})=i \frac{e \mu^{*}}{9 m_{N}} \frac{D_{T}}{\Delta} \mathrm{e}^{i \mathbf{q} \cdot \mathbf{r}_{1}} \mathbf{q} \times\left[4 \tau_{2, z} \boldsymbol{\sigma}_{2}-\left(\boldsymbol{\tau}_{1} \times \boldsymbol{\tau}_{2}\right)_{z} \boldsymbol{\sigma}_{1} \times \boldsymbol{\sigma}_{2}\right] h_{\Lambda}(r)+1 \rightleftharpoons 2,
\end{gathered}
$$

where $h_{\Lambda}(r)$ is simply the Fourier transform of the Gaussian cutoff function,

$$
h_{\Lambda}(r)=\int_{\mathbf{p}} \mathrm{e}^{-i \mathbf{p} \cdot \mathbf{r}} C_{\Lambda}(p)=\frac{\Lambda^{3}}{(4 \pi)^{3 / 2}} \mathrm{e}^{-(\Lambda r / 2)^{2}} .
$$

In the limit $\Lambda \rightarrow \infty, h_{\Lambda}(r)$ reduces to $\delta(\mathbf{r})$. In this limit, as discussed later in Sec. VII, the magnetic dipole operator derived from $\mathbf{j}_{\mathrm{h}-\mathrm{i}}^{\mathrm{N}} \mathrm{LO}(\mathbf{q})$ gives no contribution to nuclear electromagnetic observables - this follows from the antisymmetry of two-nucleon states. Smearing the $\delta$-function as in Eq. (4.13) is effectively including corrections of higher order than $\mathrm{N}^{2} \mathrm{LO}$. We shall return to this issue in Sec. VII.

\section{BEYOND N²LO: LOOP CORRECTIONS}

At $\mathrm{N}^{3} \mathrm{LO}(e Q)$, there are four classes of contributions: i) one-loop two-body currents, ii) currents from four-nucleon contact interactions involving two gradients of the nucleon fields, iii) one-loop renormalization corrections to tree-level two-body currents, and iv) $(Q / M)^{2}$ relativistic corrections to the NLO currents resulting from the non-relativistic reduction of the vertices. We now turn to a derivation of the contributions in the first two classes. Those in the last two will be derived in a later work [19]. 


\section{A. One-loop two-body currents}

In this section we consider one-loop two-body currents. Those involving pions and nucleons only in the intermediate states are illustrated by the diagrams in Fig. 6, where we show only one among all possible time orderings. Referring to this figure, we find

$$
\mathbf{p}_{1}+\mathbf{k}_{1} \quad \mathbf{p}_{2}+\mathbf{k}_{2}
$$

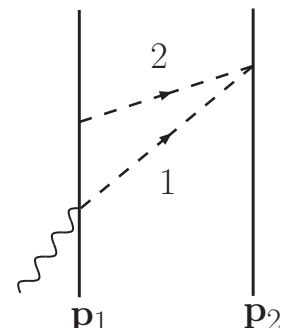

a)

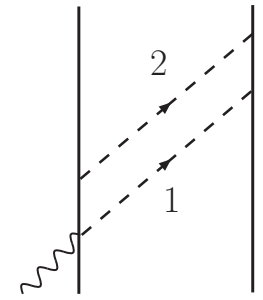

d)

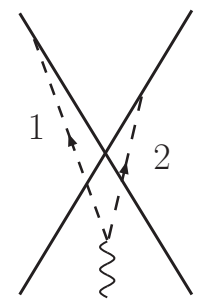

g)

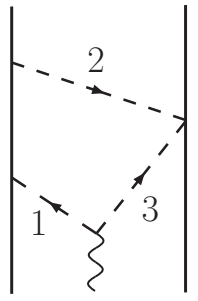

b)

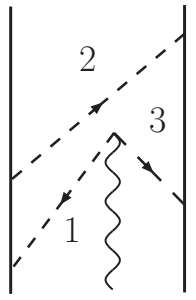

e)

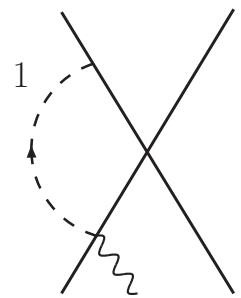

h)

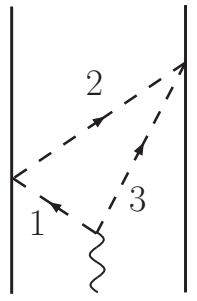

c)

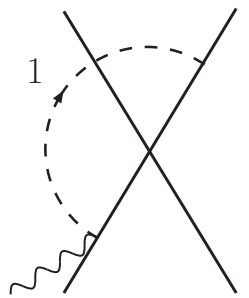

f)

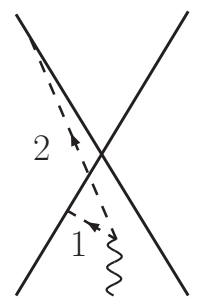

i)

FIG. 6: Diagrams illustrating one-loop two-body currents. Only one among the possible time orderings is shown. Notation is as in Fig. 2.

$$
\text { type a }=-2 i \frac{e g_{A}^{2}}{F_{\pi}^{4}} \int \frac{\tau_{2, z}\left(\boldsymbol{\sigma}_{1} \times \mathbf{q}_{2}\right)+\left(\boldsymbol{\tau}_{1} \times \boldsymbol{\tau}_{2}\right)_{z} \mathbf{q}_{2}}{\omega_{1} \omega_{2}\left(\omega_{1}+\omega_{2}\right)}+1 \rightleftharpoons 2,
$$

where the $\mathbf{q}_{i}$ 's and $\omega_{i}=\left(q_{i}^{2}+m_{\pi}^{2}\right)^{1 / 2}$ denote the momenta (with the flow as indicated in the figure) and energies of the exchanged pions, and the integration is on any one of the $\mathbf{q}_{i}$ 's, the remaining $\mathbf{q}_{j}$ 's with $j \neq i$ being fixed by momentum-conserving $\delta$-functions. Type b) diagrams give

$$
\begin{aligned}
\text { type b) } & =2 i \frac{e g_{A}^{2}}{F_{\pi}^{4}} \int \frac{\mathbf{q}_{1}-\mathbf{q}_{3}}{\omega_{1} \omega_{2} \omega_{3}} \frac{\omega_{1}+\omega_{2}+\omega_{3}}{\left(\omega_{1}+\omega_{2}\right)\left(\omega_{1}+\omega_{3}\right)\left(\omega_{2}+\omega_{3}\right)}\left[\left(\boldsymbol{\tau}_{1} \times \boldsymbol{\tau}_{2}\right)_{z} \mathbf{q}_{1} \cdot \mathbf{q}_{2}\right. \\
& \left.-\tau_{2, z} \boldsymbol{\sigma}_{1} \cdot\left(\mathbf{q}_{1} \times \mathbf{q}_{2}\right)\right]+1 \rightleftharpoons 2 .
\end{aligned}
$$


Next, the contributions of type c)-e) diagrams are:

$$
\begin{aligned}
& \text { type c })=-i \frac{e}{2 F_{\pi}^{4}}\left(\boldsymbol{\tau}_{1} \times \boldsymbol{\tau}_{2}\right)_{z} \int \frac{\mathbf{q}_{1}-\mathbf{q}_{3}}{\omega_{1} \omega_{3}} \frac{\omega_{2}\left(\omega_{1}+\omega_{2}+\omega_{3}\right)-3 \omega_{1} \omega_{3}}{\left(\omega_{1}+\omega_{2}\right)\left(\omega_{1}+\omega_{3}\right)\left(\omega_{2}+\omega_{3}\right)}, \\
& \text { type d })=-2 i \frac{e g_{A}^{4}}{F_{\pi}^{4}} \int \frac{\omega_{1}^{2}+\omega_{2}^{2}+\omega_{1} \omega_{2}}{\omega_{1}^{3} \omega_{2}^{3}\left(\omega_{1}+\omega_{2}\right)}\left[\left(\boldsymbol{\tau}_{1} \times \boldsymbol{\tau}_{2}\right)_{z} \mathbf{q}_{2}\left(\mathbf{q}_{1} \cdot \mathbf{q}_{2}\right)+2 \tau_{2, z} \mathbf{q}_{1} \cdot \mathbf{q}_{2}\left(\boldsymbol{\sigma}_{1} \times \mathbf{q}_{2}\right)\right. \\
& \left.+2 \tau_{1, z} \mathbf{q}_{2} \boldsymbol{\sigma}_{2} \cdot\left(\mathbf{q}_{1} \times \mathbf{q}_{2}\right)\right]+1 \rightleftharpoons 2 \text {, } \\
& \text { type e })=2 i \frac{e g_{A}^{4}}{F_{\pi}^{4}} \int\left(\mathbf{q}_{1}-\mathbf{q}_{3}\right) f\left(\omega_{1}, \omega_{2}, \omega_{3}\right)\left[\left(\boldsymbol{\tau}_{1} \times \boldsymbol{\tau}_{2}\right)_{z}\left(\mathbf{q}_{1} \cdot \mathbf{q}_{2}\right)\left(\mathbf{q}_{2} \cdot \mathbf{q}_{3}\right)\right. \\
& \left.+2 \tau_{2, z}\left(\mathbf{q}_{2} \cdot \mathbf{q}_{3}\right) \boldsymbol{\sigma}_{1} \cdot\left(\mathbf{q}_{2} \times \mathbf{q}_{1}\right)+2 \tau_{1, z}\left(\mathbf{q}_{1} \cdot \mathbf{q}_{2}\right) \boldsymbol{\sigma}_{2} \cdot\left(\mathbf{q}_{3} \times \mathbf{q}_{2}\right)\right]
\end{aligned}
$$

where the function $f\left(\omega_{1}, \omega_{2}, \omega_{3}\right)$ containing the pion energy factors from field normalizations and energy denominators for diagrams of type e) is defined as

$$
\begin{aligned}
f\left(\omega_{1}, \omega_{2}, \omega_{3}\right) & =\frac{1}{\omega_{1} \omega_{2} \omega_{3}\left(\omega_{1}+\omega_{2}\right)\left(\omega_{1}+\omega_{3}\right)\left(\omega_{2}+\omega_{3}\right)}\left[\frac{\omega_{1} \omega_{2}+\omega_{2} \omega_{3}+\omega_{1} \omega_{3}}{\omega_{1} \omega_{2} \omega_{3}}\right. \\
& \left.+\frac{\left(\omega_{1}+\omega_{2}\right)\left(\omega_{2}+\omega_{3}\right)\left(\omega_{1}^{2}+\omega_{3}^{2}\right)}{\omega_{1}^{2} \omega_{2} \omega_{3}^{2}}+\frac{\omega_{2}}{\omega_{1} \omega_{3}}+\frac{\omega_{1}+\omega_{2}+\omega_{3}}{\omega_{2}^{2}}\right] .
\end{aligned}
$$

Lastly, diagrams of type f) and h) vanish, since the integrand (in the static limit) is an odd function of the loop momentum $\mathbf{q}_{1}$,

$$
\text { type } \mathrm{f}) \text { and } \mathrm{h}) \propto \int \frac{\mathbf{q}_{1}}{\omega_{1}^{3}} \times(\text { spin-isospin structure }) \text {. }
$$

However, the contributions of type g) and i) diagrams read:

$$
\begin{array}{r}
\text { type g) }=2 i \frac{e g_{A}^{2} C_{T}}{F_{\pi}^{2}}\left(\boldsymbol{\tau}_{1} \times \boldsymbol{\tau}_{2}\right)_{z} \int \frac{\mathbf{q}_{1}-\mathbf{q}_{2}}{\omega_{1}^{3} \omega_{2}^{3}} \frac{\omega_{1}^{2}+\omega_{1} \omega_{2}+\omega_{2}^{2}}{\omega_{1}+\omega_{2}}\left(\boldsymbol{\sigma}_{1} \cdot \mathbf{q}_{2}\right)\left(\boldsymbol{\sigma}_{2} \cdot \mathbf{q}_{1}\right), \\
\text { type i })=-\frac{e g_{A}^{2}}{2 F_{\pi}^{2}}\left(\boldsymbol{\tau}_{1} \times \boldsymbol{\tau}_{2}\right)_{z} \int \frac{\mathbf{q}_{1}-\mathbf{q}_{2}}{\omega_{1}^{3} \omega_{2}^{3}} \frac{\omega_{1}^{2}+\omega_{1} \omega_{2}+\omega_{2}^{2}}{\omega_{1}+\omega_{2}}\left[C_{S} \boldsymbol{\sigma}_{1} \cdot\left(\mathbf{q}_{1} \times \mathbf{q}_{2}\right)\right. \\
\left.\quad+i C_{T}\left[\left(\boldsymbol{\sigma}_{1} \cdot \mathbf{q}_{2}\right)\left(\boldsymbol{\sigma}_{2} \cdot \mathbf{q}_{1}\right)+\left(\boldsymbol{\sigma}_{1} \cdot \mathbf{q}_{1}\right)\left(\boldsymbol{\sigma}_{2} \cdot \mathbf{q}_{2}\right)+i \boldsymbol{\sigma}_{2} \cdot\left(\mathbf{q}_{1} \times \mathbf{q}_{2}\right)\right]\right]+1 \rightleftharpoons 2 .
\end{array}
$$

A few comments are now in order. Firstly, the evaluation of the current operators resulting from the diagrams of Fig. 6 panels d)-e) and g) is carried out by including the recoil corrections of order $e Q$ to the reducible diagrams shown in Fig. 7 panels a)-c). As an example, we consider the irreducible and reducible contributions represented in Fig. 6 d) and Fig. 7 a), respectively. We follow the procedure adopted in Sec. IIIA, and expand, in the reducible diagrams, the energies of the intermediate nucleonic states, which are suppressed by a factor 

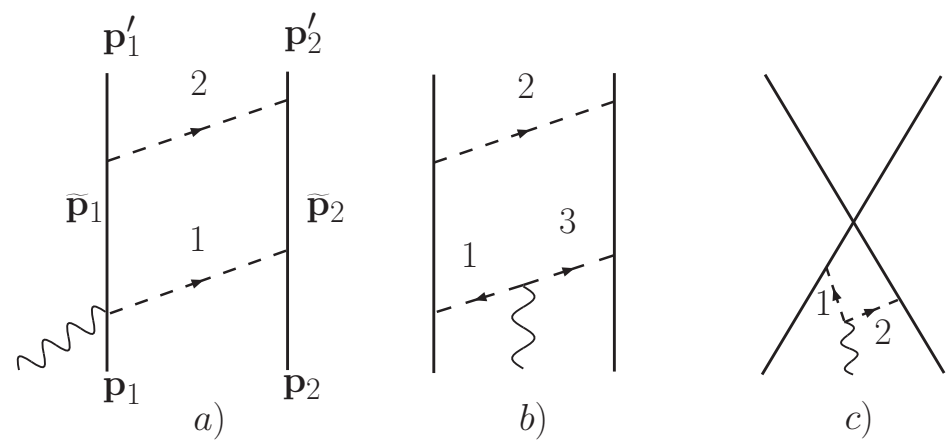

c)

FIG. 7: Diagrams illustrating the reducible one-loop two-body currents. Only one among the possible time orderings is shown. Notation is as in Fig. 2 ,

$Q / M$ with respect to the pionic energies $\omega_{i} \sim Q$. Up to order $e Q$, the current operator $\mathbf{j}_{\text {red }}$ associated with the reducible box diagrams then reads

$$
\begin{aligned}
\mathbf{j}_{\mathrm{red}} & =\int v^{\pi}\left(\mathbf{q}_{2}\right) \frac{1}{E_{i}-\widetilde{E}_{1}-\widetilde{E}_{2}+i \eta} \mathbf{j}^{\mathrm{NLO}}\left(\mathbf{q}_{1}\right) \\
& -\int 2 \frac{\omega_{1}+\omega_{2}}{\omega_{1} \omega_{2}} V_{\pi N N}\left(2, \mathbf{q}_{2}\right) V_{\pi N N}\left(2, \mathbf{q}_{1}\right) V_{\pi N N}\left(1, \mathbf{q}_{2}\right) V_{\gamma \pi N N}\left(1, \mathbf{q}_{1}\right)
\end{aligned}
$$

where $v^{\pi}\left(\mathbf{q}_{2}\right)$ and $\mathbf{j}^{\mathrm{NLO}}\left(\mathbf{q}_{1}\right)$ are the OPEP and pion-seagull current operators defined in Eqs. (3.11) and (3.3), respectively. The $V\left(i, \mathbf{q}_{j}\right)$ denotes the vertex from the interaction Hamiltonian relative to nucleon $i$ and a pion with momentum $\mathbf{q}_{j}$, and $E_{i}$ is the initial energy of the system, while $\widetilde{E}_{1}$ and $\widetilde{E}_{2}$ are the energies of the intermediate nucleons. The first term of Eq. (5.10) is then embedded in the iterated solution of the Lippmann-Schwinger equation, whereas the second term due to recoil corrections is retained and added to the irreducible contribution, $\mathbf{j}_{\mathrm{irr}}$, which is given by

$$
\begin{aligned}
\mathbf{j}_{\text {irr }} & =\int \frac{2}{\omega_{1} \omega_{2}\left(\omega_{1}+\omega_{2}\right)} V_{\pi N N}\left(2, \mathbf{q}_{2}\right) V_{\pi N N}\left(2, \mathbf{q}_{1}\right) V_{\pi N N}\left(1, \mathbf{q}_{1}\right) V_{\gamma \pi N N}\left(1, \mathbf{q}_{1}\right) \\
& +\int 2 \frac{\omega_{1}^{2}+\omega_{2}^{2}+\omega_{1} \omega_{2}}{\omega_{1} \omega_{2}\left(\omega_{1}+\omega_{2}\right)} V_{\pi N N}\left(2, \mathbf{q}_{1}\right) V_{\pi N N}\left(2, \mathbf{q}_{2}\right) V_{\pi N N}\left(1, \mathbf{q}_{2}\right) V_{\gamma \pi N N}\left(1, \mathbf{q}_{1}\right) .
\end{aligned}
$$

The first term above comes from the irreducible direct diagrams (in which, with reference to Fig. 6 d), pion 1 is absorbed before pion 2), while the second term is from the crossed diagrams (in which pion 1 is absorbed after pion 2). Equation (5.11) can be further simplified expressing the product $V_{\pi N N}\left(2, \mathbf{q}_{1}\right) V_{\pi N N}\left(2, \mathbf{q}_{2}\right)$ as

$$
V_{\pi N N}\left(2, \mathbf{q}_{1}\right) V_{\pi N N}\left(2, \mathbf{q}_{2}\right)=\left[V_{\pi N N}\left(2, \mathbf{q}_{1}\right), V_{\pi N N}\left(2, \mathbf{q}_{2}\right)\right]_{-}+V_{\pi N N}\left(2, \mathbf{q}_{2}\right) V_{\pi N N}\left(2, \mathbf{q}_{1}\right),
$$

to obtain

$$
\begin{aligned}
\mathbf{j}_{\text {irr }} & =\int 2 \frac{\omega_{1}+\omega_{2}}{\omega_{1} \omega_{2}} V_{\pi N N}\left(2, \mathbf{q}_{2}\right) V_{\pi N N}\left(2, \mathbf{q}_{1}\right) V_{\pi N N}\left(1, \mathbf{q}_{2}\right) V_{\gamma \pi N N}\left(1, \mathbf{q}_{1}\right) \\
& +\int 2 \frac{\omega_{1}^{2}+\omega_{2}^{2}+\omega_{1} \omega_{2}}{\omega_{1} \omega_{2}\left(\omega_{1}+\omega_{2}\right)}\left[V_{\pi N N}\left(2, \mathbf{q}_{1}\right), V_{\pi N N}\left(2, \mathbf{q}_{2}\right)\right]_{-} V_{\pi N N}\left(1, \mathbf{q}_{2}\right) V_{\gamma \pi N N}\left(1, \mathbf{q}_{1}\right) .
\end{aligned}
$$


The complete current of type d) is then

type d $)=\int 2 \frac{\omega_{1}^{2}+\omega_{2}^{2}+\omega_{1} \omega_{2}}{\omega_{1} \omega_{2}\left(\omega_{1}+\omega_{2}\right)}\left[V_{\pi N N}\left(2, \mathbf{q}_{1}\right), V_{\pi N N}\left(2, \mathbf{q}_{2}\right)\right]_{-} V_{\pi N N}\left(1, \mathbf{q}_{2}\right) V_{\gamma \pi N N}\left(1, \mathbf{q}_{1}\right)-$ h.c. ,

where the h.c. term corresponds to including the diagrams in which the photon hooks up to the pion with momentum $\mathbf{q}_{2}$. Note that the recoil corrections exactly cancel the first term of Eq. (5.13), leaving the term proportional to the energy factor associated with the crossed diagrams only. We find it interesting that these cancellations are also obtained for the current of type e), which reads

$$
\begin{aligned}
\text { type e }) & =\int 4 f\left(\omega_{1}, \omega_{2}, \omega_{3}\right)\left[V_{\pi N N}\left(2, \mathbf{q}_{3}\right), V_{\pi N N}\left(2, \mathbf{q}_{2}\right)\right]_{-} \\
& \times V_{\pi N N}\left(1, \mathbf{q}_{2}\right) V_{\pi N N}\left(1, \mathbf{q}_{1}\right) V_{\gamma \pi \pi}\left(\mathbf{q}_{1}, \mathbf{q}_{3}\right)-\text { h.c. },
\end{aligned}
$$

and it is therefore tempting to conjecture that they persist at higher orders. However, this statement has not been proven.

Secondly, we observe that diagrams of the type shown in Fig. 8 are suppressed by an extra power of $Q$ relative to those considered in this section, i.e. they are of order e $Q^{2}$. For example, the diagrams of type a) give rise to the following current operator

$$
\text { type a) in Fig. } 8=\frac{e}{m} \frac{g_{A}^{2}}{F_{\pi}^{4}} \tau_{z, 1}\left(2 \mathbf{K}_{1}+i \boldsymbol{\sigma}_{1} \times \mathbf{k}_{1}\right) \int \frac{\mathbf{q}_{1} \cdot \mathbf{q}_{2}}{\omega_{1}^{2} \omega_{2}^{2}}+1 \rightleftharpoons 2,
$$

where the momentum $\mathbf{K}_{i}$ is as given in Eq. (3.1), while those of type b) vanish, since they are proportional $\left(\delta_{a z} \tau_{1, b}+\delta_{b z} \tau_{1, a}-2 \delta_{a b} \tau_{1, z}\right) \epsilon_{a b c} \tau_{2, c}=\left(\epsilon_{z b c}+\epsilon_{b z c}\right) \tau_{1, b} \tau_{2, c}$.

Lastly, as a check, we have re-derived the nucleon-nucleon potential at the one-loop level (both with and without the inclusion of explicit $\Delta$-isobar degrees of freedom), and have explicitly verified that it is in agreement with that obtained in Refs. [2] and [3]. In particular, we note that if recoil corrections to the reducible diagrams, for example box diagrams, are retained along with the contributions of irreducible diagrams, the resulting potential is in agreement with that derived in Ref. [3] with the method of unitary transformations (in this respect, see Sec. VI).

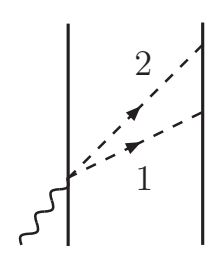

a)

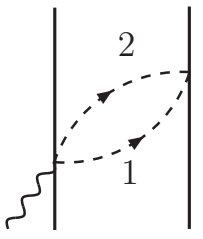

b)

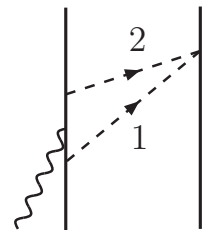

c)

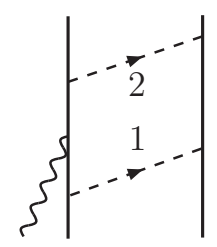

d)

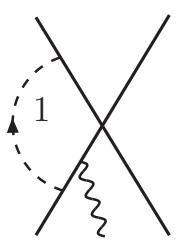

e)

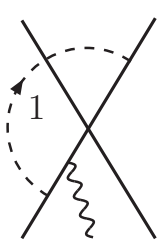

f)

FIG. 8: Diagrams illustrating $\mathrm{N}^{4} \mathrm{LO}$ contributions not included in the present work. Only one among the possible time orderings is shown. Notation is as in Fig. 2.

We conclude this section by showing in Figs. 9 and 10 the one-loop two-body currents involving one- and two- $\Delta$ intermediate states. A listing of the explicit expressions is given in Appendix C. 
$\mathbf{p}_{1}+\mathbf{k}_{1} \quad \mathbf{p}_{2}+\mathbf{k}_{2}$
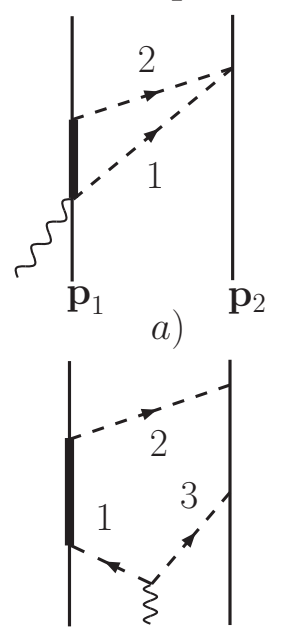

e)

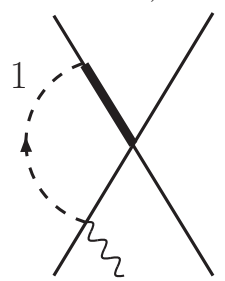

i)

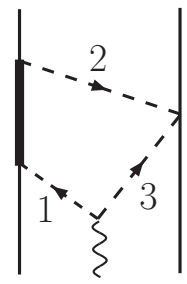

b)

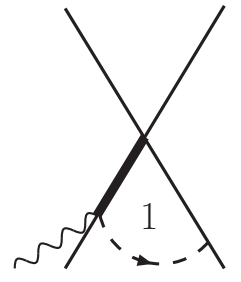

f)

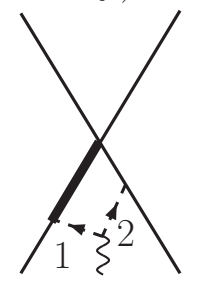

j)

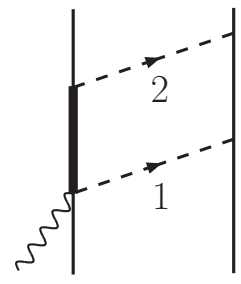

c)

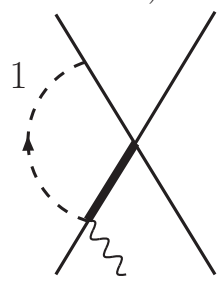

g)

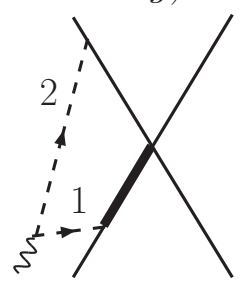

$k)$

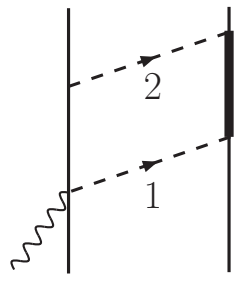

d)

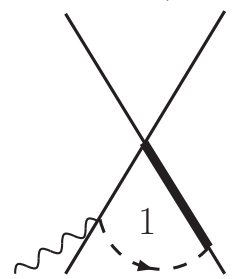

h)

FIG. 9: Diagrams illustrating one-loop two-body currents with a single $\Delta$ isobar in the intermediate states. Only one among the possible time orderings is shown. Thin, thick, dashed, and wavy lines denote nucleons, $\Delta$ isobars, pions, and photons, respectively.

\section{B. Currents from four-nucleon contact interactions}

In this section we report the $\mathrm{N}^{3} \mathrm{LO}$ contributions to the current operator from the contact electromagnetic interactions of Eqs. (D11)-(D19). We find:

$$
\begin{aligned}
\mathbf{j}_{\mathrm{CT} \gamma}^{\mathrm{N}^{3} \mathrm{LO}} & =-e e_{1}\left[2\left(2 C_{1}^{\prime}-C_{2}^{\prime}\right) \mathbf{K}_{2}+4 C_{3}^{\prime} \mathbf{K}_{1}+i C_{4}^{\prime}\left(\boldsymbol{\sigma}_{1}+\boldsymbol{\sigma}_{2}\right) \times \mathbf{k}_{2}+i C_{5}^{\prime} \boldsymbol{\sigma}_{1} \times \mathbf{k}_{1}\right. \\
& -i C_{6}^{\prime} \boldsymbol{\sigma}_{2} \times \mathbf{k}_{1}+2\left(2 C_{7}^{\prime}-C_{10}^{\prime}\right)\left(\mathbf{K}_{2} \cdot \boldsymbol{\sigma}_{2}\right) \boldsymbol{\sigma}_{1}+2\left(2 C_{8}^{\prime}-C_{11}^{\prime}\right)\left(\mathbf{K}_{2} \cdot \boldsymbol{\sigma}_{1}\right) \boldsymbol{\sigma}_{2} \\
& -2 C_{13}^{\prime}\left[\left(\mathbf{K}_{1} \cdot \boldsymbol{\sigma}_{1}\right) \boldsymbol{\sigma}_{2}+\left(\mathbf{K}_{1} \cdot \boldsymbol{\sigma}_{2}\right) \boldsymbol{\sigma}_{1}\right]+2\left(2 C_{9}^{\prime}-C_{12}^{\prime}\right) \mathbf{K}_{2}\left(\boldsymbol{\sigma}_{1} \cdot \boldsymbol{\sigma}_{2}\right) \\
& \left.-4 C_{14}^{\prime} \mathbf{K}_{1}\left(\boldsymbol{\sigma}_{1} \cdot \boldsymbol{\sigma}_{2}\right)\right]+1 \rightleftharpoons 2 .
\end{aligned}
$$

Again, we note that the nucleon-nucleon potential generated by the contact interactions of Eqs. (D2)-(D10) is in agreement with that obtained in Ref. [3]. 


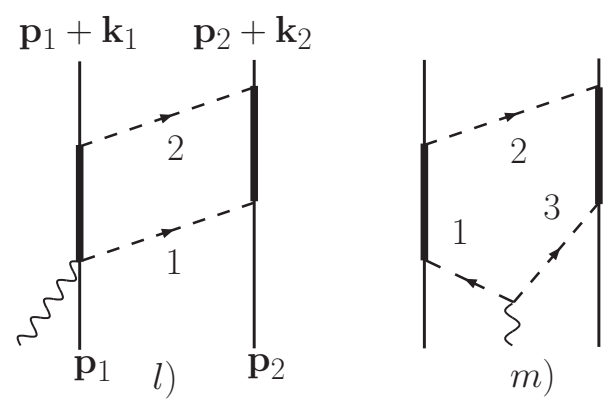

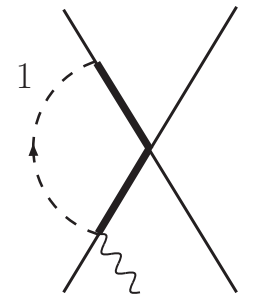

$n)$

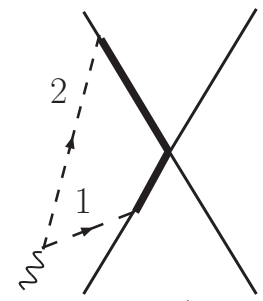

q)

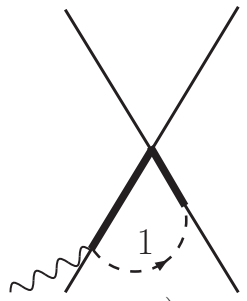

O)

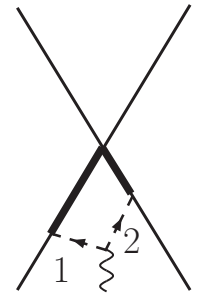

$r)$

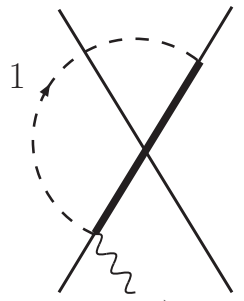

p)

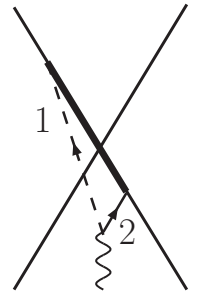

$s)$

FIG. 10: Diagrams illustrating one-loop two-body currents with two $\Delta$ isobars in the intermediate states. Only one among the possible time orderings is shown. Notation is as in Fig. 9 ,

\section{CURRENT CONSERVATION UP TO N ${ }^{3}$ LO}

The nuclear electromagnetic current operator is related to the Hamiltonian through the continuity equation, which in the momentum space reads

$$
\mathbf{q} \cdot \mathbf{j}=\left[\frac{p_{1}^{2}}{2 m_{N}}+\frac{p_{2}^{2}}{2 m_{N}}+v_{12}, \rho\right]_{-},
$$

where $[\ldots, \ldots]_{-}$denotes the commutator, $\mathbf{q}$ is momentum transfer, and $\rho$ is the charge operator given to LO, in the notation of Eq. (2.30), by

$$
\rho=e\left(e_{1}+e_{2}\right) \text {. }
$$

It is well known (and easily verified) that the LO and NLO currents satisfy the continuity equation with, respectively, the kinetic energy terms and the LO $\left(Q^{0}\right)$ contribution to the potential, i.e. OPEP. The $\mathrm{N}^{2} \mathrm{LO}$ currents involving $\Delta$ excitation are purely transverse, and therefore do not enter the continuity equation, while those arising from relativistic corrections to the LO one-body term require the inclusion of these corrections also in the charge operator, in order for the continuity equation to be satisfied. We will not discuss them further here. 


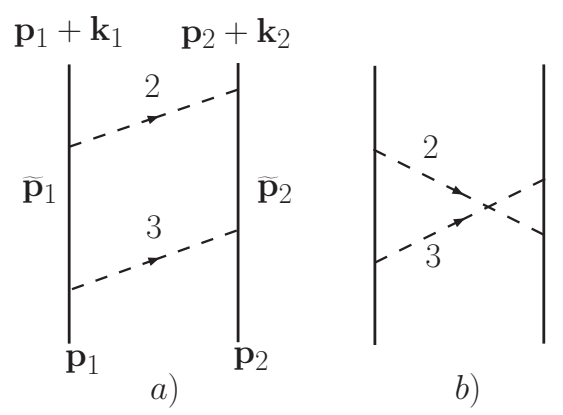

FIG. 11: Diagrams illustrating the reducible, panel a), and irreducible, panel b), two-body box potential. Only one among the possible time orderings is shown. Notation is as in Fig. 2 .

As an internal check, we have explicitly verified that the $\mathrm{N}^{3} \mathrm{LO}$ current operators obtained in the previous section satisfy the continuity equation with the $\mathrm{N}^{2} \mathrm{LO}\left(Q^{2}\right)$ contributions to the potential, induced by the interaction Hamiltonians given in Sec. II A and Appendix D 1 . For the purpose of illustration, we give more details on the calculation carried out for the currents of type d) and e) of Fig. 6. The potential generated by the box diagrams shown in Fig. 11 is given by

$$
v_{\text {box }}=-\frac{g_{A}^{4}}{F_{\pi}^{4}} \int \frac{\omega_{2}^{2}+\omega_{3}^{2}+\omega_{2} \omega_{3}}{\omega_{2}^{3} \omega_{3}^{3}\left(\omega_{2}+\omega_{3}\right)}\left[2 \boldsymbol{\tau}_{1} \cdot \boldsymbol{\tau}_{2}\left(\mathbf{q}_{2} \cdot \mathbf{q}_{3}\right)^{2}+3 \boldsymbol{\sigma}_{1} \cdot\left(\mathbf{q}_{2} \times \mathbf{q}_{3}\right) \boldsymbol{\sigma}_{2} \cdot\left(\mathbf{q}_{2} \times \mathbf{q}_{3}\right)\right],
$$

where $\mathbf{q}_{2}+\mathbf{q}_{3}=\mathbf{k}_{2}$ or $-\mathbf{k}_{1}$. We note that the recoil corrections to the reducible box diagrams have been included, consistently with our treatment in the previous section.

Evaluation of the commutator of $v_{\text {box }}$ with the charge operator gives

$$
\left[v_{\text {box }}, \rho\right]=-i e \frac{2 g_{A}^{4}}{F_{\pi}^{4}}\left(\boldsymbol{\tau}_{1} \times \boldsymbol{\tau}_{2}\right)_{z} \int \frac{\omega_{2}^{2}+\omega_{3}^{2}+\omega_{2} \omega_{3}}{\omega_{2}^{3} \omega_{3}^{3}\left(\omega_{2}+\omega_{3}\right)}\left(\mathbf{q}_{2} \cdot \mathbf{q}_{3}\right)^{2}+1 \rightleftharpoons 2,
$$

where $\mathbf{k}_{1}+\mathbf{k}_{2}=\mathbf{q}$ and $\mathbf{q}_{2}+\mathbf{q}_{3}=\mathbf{k}_{2}$. On the other hand, the l.h.s. of Eq. (6.1) for the currents of type d) and e) in Fig. 6 reads

$$
\begin{aligned}
& \mathbf{q} \cdot \mathbf{j}_{\mathrm{d}}=-i e \frac{2 g_{A}^{4}}{F_{\pi}^{4}} \int \frac{\omega_{2}^{2}+\omega_{3}^{2}+\omega_{2} \omega_{3}}{\omega_{2}^{3} \omega_{3}^{3}\left(\omega_{2}+\omega_{3}\right)}\left[\left(\boldsymbol{\tau}_{1} \times \boldsymbol{\tau}_{2}\right)_{z} \mathbf{q}_{2}\left(\mathbf{q}_{2} \cdot \mathbf{q}_{3}\right)+2 \tau_{2, z}\left(\mathbf{q}_{2} \cdot \mathbf{q}_{3}\right)\left(\boldsymbol{\sigma}_{1} \times \mathbf{q}_{2}\right)\right. \\
&+\left.2 \tau_{1, z} \mathbf{q}_{2} \boldsymbol{\sigma}_{2} \cdot\left(\mathbf{q}_{3} \times \mathbf{q}_{2}\right)\right] \cdot \mathbf{q}+1 \rightleftharpoons 2 \\
& \mathbf{q} \cdot \mathbf{j}_{\mathrm{e}}=i e \frac{2 g_{A}^{4}}{F_{\pi}^{4}} \int\left[\frac{\omega_{2}^{2}+\omega_{3}^{2}+\omega_{2} \omega_{3}}{\omega_{2}^{3} \omega_{3}^{3}\left(\omega_{2}+\omega_{3}\right)}-\frac{\omega_{1}^{2}+\omega_{2}^{2}+\omega_{1} \omega_{2}}{\omega_{1}^{3} \omega_{2}^{3}\left(\omega_{1}+\omega_{2}\right)}\right]\left[\left(\boldsymbol{\tau}_{1} \times \boldsymbol{\tau}_{2}\right)_{z}\left(\mathbf{q}_{1} \cdot \mathbf{q}_{2}\right)\left(\mathbf{q}_{2} \cdot \mathbf{q}_{3}\right)\right. \\
&\left.+2 \tau_{2, z}\left(\mathbf{q}_{2} \cdot \mathbf{q}_{3}\right) \boldsymbol{\sigma}_{1} \cdot\left(\mathbf{q}_{2} \times \mathbf{q}_{1}\right)+2 \tau_{1, z}\left(\mathbf{q}_{1} \cdot \mathbf{q}_{2}\right) \boldsymbol{\sigma}_{2} \cdot\left(\mathbf{q}_{3} \times \mathbf{q}_{2}\right)\right],
\end{aligned}
$$

where $\mathbf{q}_{1}=\mathbf{q}_{2}+\mathbf{k}_{1}$, and the factor $\mathbf{q} \cdot\left(\mathbf{q}_{1}-\mathbf{q}_{3}\right) f\left(\omega_{1}, \omega_{2}, \omega_{3}\right)=\left(\omega_{1}^{2}-\omega_{3}^{2}\right) f\left(\omega_{1}, \omega_{2}, \omega_{3}\right)$ has been written as in the square brackets of the last equation. Combining Eqs. (6.5) -(6.6)), we obtain

$$
\begin{aligned}
& \mathbf{q} \cdot\left(\mathbf{j}_{\mathrm{d}}+\mathbf{j}_{\mathrm{e}}\right)=-i e \frac{2 g_{A}^{4}}{F_{\pi}^{4}} \int \frac{\omega_{2}^{2}+\omega_{3}^{2}+\omega_{2} \omega_{3}}{\omega_{2}^{3} \omega_{3}^{3}\left(\omega_{2}+\omega_{3}\right)}\left[\left(\boldsymbol{\tau}_{1} \times \boldsymbol{\tau}_{2}\right)_{z}\left(\mathbf{q}_{2} \cdot \mathbf{q}_{3}\right)^{2}\right. \\
& \left.\quad+2 \tau_{2, z}\left(\mathbf{q}_{2} \cdot \mathbf{q}_{3}\right) \boldsymbol{\sigma}_{1} \cdot\left(\mathbf{q}_{2} \times \mathbf{q}_{3}\right)+2 \tau_{1, z}\left(\mathbf{q}_{2} \cdot \mathbf{q}_{3}\right) \boldsymbol{\sigma}_{2} \cdot\left(\mathbf{q}_{3} \times \mathbf{q}_{2}\right)\right]+1 \rightleftharpoons 2
\end{aligned}
$$


and the last two terms of the previous equation vanish. This is easily seen by changing $\mathbf{q}_{2} \rightarrow \mathbf{k}_{2} / 2+\mathbf{q}_{2}$ (implying $\mathbf{q}_{3}=\mathbf{k}_{2} / 2-\mathbf{q}_{2}$ ), and observing that the integrands are odd under $\mathbf{q}_{2} \rightarrow-\mathbf{q}_{2}$. Hence we are left with the first term which is equal to Eq. (6.4), showing that the continuity equation is indeed satisfied.
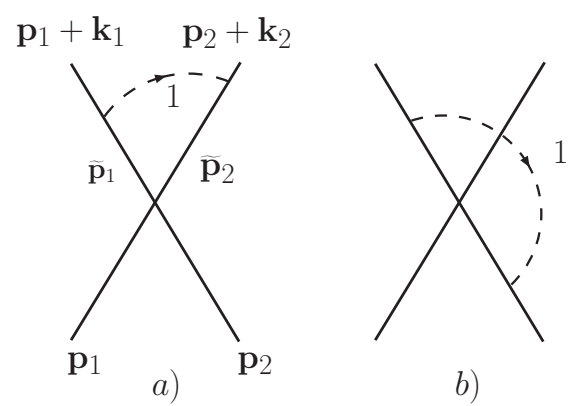

FIG. 12: Diagrams illustrating the reducible, panel a), and irreducible, panel b), two-body one-loop contact potential. Only one among the possible time orderings is shown. Notation is as in Fig. 2 ,

Two closing remarks are in order. First, ignoring the recoil corrections in both the potential and currents leads to a violation of the continuity equation. This remains valid also for the current of type g) and the potential $v_{\mathrm{ct}}$, generated by the diagrams shown in Fig. 12 and given explicitly by

$$
v_{\mathrm{ct}}=\frac{4}{3} \frac{g_{A}^{2}}{F_{\pi}^{2}} \boldsymbol{\tau}_{1} \cdot \boldsymbol{\tau}_{2} \boldsymbol{\sigma}_{1} \cdot \boldsymbol{\sigma}_{2} \int \frac{\mathbf{q}_{1}^{2}}{\omega_{1}^{3}} .
$$

Second, in hybrid calculations, such as those reported below, current conservation is not strictly satisfied. Assuming, however, that differences between the $\chi \mathrm{EFT}$ and realistic potentials occur at orders higher than $\mathrm{N}^{2} \mathrm{LO}$, the $\mathrm{N}^{3} \mathrm{LO}$ currents derived here are then approximately conserved.

\section{ELECTROMAGNETIC OBSERVABLES AT N ${ }^{2}$ LO IN $A=2-3$ SYSTEMS}

We present results obtained for a number of low-energy electromagnetic observables in the $A=2$ and 3 nuclei using the current operators derived at $\mathrm{N}^{2} \mathrm{LO}$. In the tables to follow, we denote respectively with $\mathrm{LO}, \mathrm{NLO}, \mathrm{N}^{2} \mathrm{LO}-\mathrm{RC}, \mathrm{N}^{2} \mathrm{LO}-\Delta$, and $\mathrm{N}^{2} \mathrm{LO}-\Delta_{c}$ the contributions calculated with the one-body current, or impulse-approximation (IA), of Eq. (4.4), the onepion exchange two-body current at tree level of Eqs. (4.6)-(4.7), the relativistic correction to the LO current of Eq. (4.11), the single $\Delta$-isobar excitation current of Eq. (4.12), and lastly the two-body current of Eq. (4.13), due to the contact $(N N)(\Delta N)$ interaction. In the long-wavelength limit of interest in the present work, the LO and $\mathrm{N}^{2} \mathrm{LO}-\mathrm{RC}$ currents are completely determined by the experimental values of the proton and neutron magnetic moments, respectively +2.793 and -1.913 in units of nuclear magnetons (n.m.). The NLO current involves the combination $g_{A} / F_{\pi}$, for which we adopt the value $\left(m_{\pi} g_{A} / F_{\pi}\right)^{2} /(4 \pi)=0.075$ as inferred from the Nijmegen analysis of nucleon-nucleon elastic scattering data [26]. The $N \Delta$ transition magnetic moment is taken to be $\mu^{*}=3 \mathrm{n} . \mathrm{m}$. from an analysis of $\gamma N$ data at resonance [27]. The coupling constant $h_{A}$ in the $\mathrm{N}^{2} \mathrm{LO}-\Delta$ term is fixed by reproducing the 
width of the $\Delta$ resonance, $h_{A}=2.191 g_{A}$ and $g_{A}=1.267$, while the (unknown) coupling constant $D_{T}$ in the $\mathrm{N}^{2} \mathrm{LO}-\Delta_{c}$ term is expressed as

$$
D_{T}=f^{\prime} \frac{g_{A} h_{A}}{F_{\pi}^{2}},
$$

and the parameter $f^{\prime}$ is determined as described below.

In order to have a realistic estimate of the model dependence of the results, we use cutoff values $\Lambda$ in the range $500-800 \mathrm{MeV}$ and wave functions corresponding to two different nuclear Hamiltonians. The wave functions for $A=2$ are derived from solutions of the Schrödinger equation with the Argonne $v_{18}$ (AV18) [20] or CD-Bonn (CDB) [21] twonucleon potentials, while those for $A=3$ are obtained with the hyperspherical harmonics $(\mathrm{HH})$ expansion method (see Ref. [10] and references therein) from a Hamiltonian including, in addition to the AV18 or CDB two-nucleon, also a three-nucleon potential, the Urbana-IX (UIX) model [22]. The AV18/UIX and CDB/UIX* combinations both reproduce the experimental ${ }^{3} \mathrm{H}$ binding energy. The former also reproduces most of the measured low-energy $N-d$ scattering observables [10], with the notable exception of the vector analyzing power in $\mathrm{N}$-d elastic scattering. Unfortunately, $\mathrm{HH}$ continuum wave functions for the CDB/UIX* combination are not yet available. The UIX* model [23] is a slightly modified version of the original UIX [22] (in the UIX*, the parameter $U_{0}$ of the central repulsive term has been reduced by the factor 0.812 ).

We consider the following two- and three-nucleon observables: the ${ }^{1} \mathrm{H}(n, \gamma)^{2} \mathrm{H}$ cross section at thermal energies, the deuteron magnetic moment, the isoscalar and isovector combinations of the trinucleon magnetic moments, the cross section and photon circular polarization parameter $R_{c}$ measured in the radiative capture of (polarized, in the case of $R_{c}$ ) neutrons on deuterons at thermal energies. At $\mathrm{N}^{2} \mathrm{LO}$ there are no three-body currents. We also observe that at this order the only isoscalar terms are from the (one-body) $\mathrm{LO}$ and $\mathrm{N}^{2} \mathrm{LO}$-RC operators, which are independent of the cutoff $\Lambda$. In Tables 【1 and 【II we list

\begin{tabular}{c|c|c}
\hline \hline & AV18 & CDB \\
\hline LO & 0.8469 & 0.8521 \\
$\mathrm{~N}^{2} \mathrm{LO}-\mathrm{RC}$ & -0.0082 & -0.0080 \\
\hline \hline
\end{tabular}

TABLE II: Contributions in n.m. to the deuteron magnetic moment, obtained with the AV18 and CDB potential models. The experimental value is 0.8574 n.m..

\begin{tabular}{c|c|c}
\hline \hline & AV18/UIX & CDB/UIX \\
\hline LO & +0.4104 & +0.4183 \\
$\mathrm{~N}^{2} \mathrm{LO}-\mathrm{RC}$ & -0.0045 & -0.0052 \\
\hline \hline
\end{tabular}

TABLE III: Contributions in n.m. to the isoscalar combination of the trinucleon magnetic moments, obtained with the AV18/UIX and CDB/UIX* Hamiltonian models. The experimental value is 0.4257 n.m..

their contributions to the deuteron magnetic moment and isoscalar combination of the ${ }^{3} \mathrm{He}$ and ${ }^{3} \mathrm{H}$ magnetic moments. The $\mathrm{N}^{2} \mathrm{LO}-\mathrm{RC}$ correction is (in magnitude) about $1 \%$ of the $\mathrm{LO}$ contribution but of opposite sign, so that its inclusion increases the difference between 
the measured and calculated values. As a result, the experimental deuteron and trinucleon isoscalar magnetic moments are underpredicted by theory at the (1.6-2.1)\% and (3.0-4.7)\% levels, respectively, depending on whether the CDB and CDB/UIX* or AV18 and AV18/UIX combinations are adopted in the $A=2$ and $A=3$ calculations. However, a recent calculation of these same observables [17], based on variational Monte Carlo (VMC) wave functions corresponding to the AV18/UIX Hamiltonian, finds the magnitude of the $\mathrm{N}^{2} \mathrm{LO}-\mathrm{RC}$ correction somewhat smaller in $A=2$ ( -0.0069 n.m.) and significantly larger in $A=3$ ( -0.012 n.m.) than obtained here. The magnetic moment operator is derived via

$$
\boldsymbol{\mu}=-\frac{i}{2} \nabla_{q} \times\left.\mathbf{j}(\mathbf{q})\right|_{q=0},
$$

and from Eq. (4.11) the $\mathrm{N}^{2} \mathrm{LO}-\mathrm{RC}$ term follows as

$$
\boldsymbol{\mu}_{\mathrm{RC}}^{\mathrm{N}^{2} \mathrm{LO}}=-\frac{e}{8 m_{N}^{3}} \sum_{i=1}^{A}\left[\left[p_{i}^{2}, e_{N, i} \mathbf{L}_{i}+\mu_{N, i} \boldsymbol{\sigma}_{i}\right]_{+}+e_{N, i} \mathbf{p}_{i} \times\left(\boldsymbol{\sigma}_{i} \times \mathbf{p}_{i}\right)\right],
$$

where $\mathbf{p}_{i}=-i \nabla_{i}$ and $\mathbf{L}_{i}$ are the linear momentum and angular momentum operators of particle $i$, and $[\ldots, \ldots]_{+}$denotes the anticommutator. The expression for this correction is different from that given in Ref. [17], which is, in turn, different from that listed in an earlier work [18] by some of the same authors of Ref. [17]. When compared to Eq. (17.3) above, for example, the term with the anticommutator is missing in Ref. [18], since the authors of that work normalize the spinors as $\bar{u} u=1$ rather than $u^{\dagger} u=1$ as in the present work. These differences might partly explain the different contributions calculated here and in Ref. [17] for the $\mathrm{N}^{2} \mathrm{LO}-\mathrm{RC}$ correction.

Next, we examine the ${ }^{1} \mathrm{H}(n, \gamma)^{2} \mathrm{H}$ radiative capture at thermal neutron energies. Various aspects of the calculations, which will not be discussed here, were reviewed most recently in Ref. [28]. The calculated values for the cross section are listed in Table IV. As remarked

\begin{tabular}{c|c|c|c||c|c|c} 
& \multicolumn{3}{|c}{ AV18 } & \multicolumn{3}{c}{ CDB } \\
\hline \hline$\Lambda(\mathrm{MeV})$ & 500 & 600 & 800 & 500 & 600 & 800 \\
\hline $\mathrm{LO}$ & 304.6 & 304.6 & 304.6 & 306.6 & 306.6 & 306.6 \\
$\mathrm{NLO}$ & 319.1 & 319.6 & 319.9 & 321.3 & 321.7 & 321.9 \\
$\mathrm{~N}^{2} \mathrm{LO}-\mathrm{RC}$ & 317.4 & 317.9 & 318.2 & 319.9 & 320.3 & 320.5 \\
$\mathrm{~N}^{2} \mathrm{LO}-\Delta$ & 321.9 & 323.8 & 326.3 & 323.8 & 325.3 & 327.1 \\
\hline \hline
\end{tabular}

TABLE IV: Cumulative contributions in mb to the ${ }^{1} \mathrm{H}(n, \gamma)^{2} \mathrm{H}$ cross section at thermal neutron energy, obtained with the AV18 and CDB potential models and cutoff values in the range 500-800 $\mathrm{MeV}$. The $\mathrm{LO}$ and $\mathrm{N}^{2} \mathrm{LO}-\mathrm{RC}$ contributions are cutoff independent, while the matrix element of the $\mathrm{N}^{2} \mathrm{LO}-\Delta_{c}$ operator vanishes. The experimental value is $332.6(0.7) \mathrm{mb}$ from Ref. [5].

earlier in Sec. IV, we note that the $\mathrm{N}^{2} \mathrm{LO}-\Delta_{c}$ current does not contribute, since the associated magnetic moment operator,

$$
\boldsymbol{\mu}_{\Delta_{c}}^{\mathrm{N}^{2} \mathrm{LO}}=-\frac{2 e \mu^{*}}{9 m_{N}} \frac{D_{T}}{\Delta} \sum_{i<j=1}^{A}\left[2\left(\tau_{i, z} \boldsymbol{\sigma}_{i}+\tau_{j, z} \boldsymbol{\sigma}_{j}\right)-\left(\boldsymbol{\tau}_{i} \times \boldsymbol{\tau}_{j}\right)_{z} \boldsymbol{\sigma}_{i} \times \boldsymbol{\sigma}_{j}\right] h_{\Lambda}\left(r_{i j}\right),
$$

is readily seen to vanish when acting on the ${ }^{1} \mathrm{~S}_{0} n$ - $p$ state [4]. If $P_{i j}^{r}, P_{i j}^{\sigma}$, and $P_{i j}^{\tau}$ denote respectively the space, spin, and isospin exchange operators, where

$$
P_{i j}^{\sigma}=\frac{1+\boldsymbol{\sigma}_{i} \cdot \boldsymbol{\sigma}_{j}}{2},
$$


and similarly for $P_{i j}^{\tau}$, then $P_{i j}^{r} P_{i j}^{\sigma} P_{i j}^{\tau}=-1$ for a two-nucleon state. The identity

$$
\boldsymbol{\sigma}_{i} \times \boldsymbol{\sigma}_{j}=i\left(\boldsymbol{\sigma}_{i}-\boldsymbol{\sigma}_{j}\right) P_{i j}^{\sigma},
$$

and the analogous one for $\boldsymbol{\tau}_{i} \times \boldsymbol{\tau}_{j}$ allow one to express the magnetic dipole operator in Eq. (7.4) as

$$
\boldsymbol{\mu}_{\Delta_{c}}^{\mathrm{N}^{2} \mathrm{LO}}=-\frac{2 e \mu^{*}}{9 m_{N}} \frac{D_{T}}{\Delta} \sum_{i<j=1}^{A} h_{\Lambda}\left(r_{i j}\right)\left[2\left(\tau_{i, z} \boldsymbol{\sigma}_{i}+\tau_{j, z} \boldsymbol{\sigma}_{j}\right)-\left(\tau_{i, z}-\tau_{j, z}\right)\left(\boldsymbol{\sigma}_{i}-\boldsymbol{\sigma}_{j}\right) P_{i j}^{r}\right] .
$$

When acting on a two-nucleon state of even relative orbital angular momentum, the square bracket in the equation above reduces to $\left(\tau_{i, z}+\tau_{j, z}\right)\left(\boldsymbol{\sigma}_{i}+\boldsymbol{\sigma}_{j}\right)$, and therefore vanishes, since this state will have either $S=0$ and $T=1$ or $S=1$ and $T=0$. Indeed, in the limit $h_{\Lambda}\left(r_{i j}\right) \rightarrow \delta\left(\mathbf{r}_{i j}\right)$ the operator in Eq. (7.4) gives no contribution. It is in this sense that one can interpret contributions at finite $\Lambda$ as representing corrections beyond $\mathrm{N}^{2} \mathrm{LO}$.

The cutoff dependence and the different short-range behaviors of the AV18 and CDB wave functions lead to a cross section of $(324.5 \pm 2.6) \mathrm{mb}$. Thus, at $\mathrm{N}^{2} \mathrm{LO}$ the experimental value, $(332.6 \pm 0.7) \mathrm{mb}[5]$, is underpredicted by roughly $2.5 \%$. The LO and NLO contributions calculated here are in agreement with those obtained for the AV18 in Refs. [17] — which uses the same form for the cutoff function - up to tiny differences presumably due to numerics. The $\mathrm{N}^{2} \mathrm{LO}-\mathrm{RC}$ contribution, however, is found to be considerably larger (in magnitude) here than in Ref. [17], although both studies predict the same sign for it, opposite to the LO contribution. Reference [17] suggests that corrections from current operators at $\mathrm{N}^{3} \mathrm{LO}$ might resolve the present discrepancy between experiment and theory (at $\mathrm{N}^{2} \mathrm{LO}$ ), and possibly reduce the model dependence in the latter. This issue will be investigated in future work. Instead, rather than using the $\Delta$ width and $\gamma-N$ data to fix the values of the coupling constants $h_{A}$ and $\mu^{*}$ entering the $\mathrm{N}^{2} \mathrm{LO}-\Delta$ current, Eq. (4.12), we determine the combination $\mu^{*} h_{A}$ by reproducing the $n$ - $p$ radiative capture cross section. In fact, we make the replacement $\mu^{*} h_{A} \rightarrow f \mu^{*} h_{A}$, take $\mu^{*}=3 \mathrm{n} . \mathrm{m}$. and $h_{A}=2.191 g_{A}$ (as before) and fix $f$ accordingly. The latter is listed in Table $\nabla$ for the various combinations of potentials and cutoffs considered

\begin{tabular}{c|c|c}
\hline \hline$\Lambda(\mathrm{MeV})$ & AV18 & CDB \\
\hline 500 & 3.352 & 3.234 \\
600 & 2.471 & 2.447 \\
800 & 1.772 & 1.814 \\
\hline \hline
\end{tabular}

TABLE V: The parameter $f$ required to reproduce, for a given value of the cutoff $\Lambda$, the experimental ${ }^{1} \mathrm{H}(n, \gamma)^{2} \mathrm{H}$ cross section at thermal neutron energy with the AV18 or CDB potential models. See text for notation.

in this work. The resulting $\mathrm{N}^{2} \mathrm{LO}$ contribution becomes then as large as the NLO - a somewhat unsettling feature of the present procedure. At NLO there is a significant cancellation between the contributions of the seagull, Eq. (4.6), and pion-in-flight, Eq. (4.7), currents. This destructive interference persists also for the three-nucleon observables considered next. Lastly, it is worth noting that conventional calculations of the ${ }^{1} \mathrm{H}(n, \gamma)^{2} \mathrm{H}$ cross section based on two-body currents constructed to satisfy current conservation with the potential used to generate the wave functions, accurately reproduce the measured value [9].

Results for the isovector combination of the trinucleon magnetic moments are presented in Table VI. Note that the row labeled $\mathrm{N}^{2} \mathrm{LO}-\Delta$ lists the contributions obtained with 
the strength of the $\mathrm{N}^{2} \mathrm{LO}-\Delta$ current determined as in Table $\mathrm{V}$. Consequently, they are significantly larger (in magnitude) and much less cutoff-dependent than those at NLO. The NLO contribution calculated in Ref. [17] with VMC wave functions and a cutoff of $600 \mathrm{MeV}$

\begin{tabular}{c|c|c|c||c|c|c} 
& \multicolumn{3}{|c}{ AV18/UIX } & \multicolumn{3}{c}{ CDB/UIX* } \\
\hline \hline$\Lambda(\mathrm{MeV})$ & 500 & 600 & 800 & 500 & 600 & 800 \\
\hline $\mathrm{LO}$ & -2.159 & -2.159 & -2.159 & -2.180 & -2.180 & -2.180 \\
$\mathrm{NLO}$ & -0.156 & -0.197 & -0.238 & -0.113 & -0.156 & -0.200 \\
$\mathrm{~N}^{2} \mathrm{LO}-\mathrm{RC}$ & +0.029 & +0.029 & +0.029 & +0.024 & +0.024 & +0.024 \\
$\mathrm{~N}^{2} \mathrm{LO}-\Delta$ & -0.258 & -0.253 & -0.250 & -0.205 & -0.202 & -0.200 \\
\hline Sum & -2.544 & -2.580 & -2.618 & -2.474 & -2.514 & -2.556 \\
\hline \hline
\end{tabular}

TABLE VI: Contributions in units of n.m. to the isovector combination of the trinucleon magnetic moments, obtained with the AV18/UIX and CDB/UIX* Hamiltonian models and cutoff values in the range $500-800 \mathrm{MeV}$. The $\mathrm{LO}$ and $\mathrm{N}^{2} \mathrm{LO}-\mathrm{RC}$ contributions are cutoff independent. The experimental value is -2.553 n.m..

is -0.205 n.m., which is $4 \%$ larger than obtained here. This is most likely due to differences in the wave functions (we note, incidentally, that VMC wave functions are less accurate than $\mathrm{HH}$ ones). We determine the strength of the $\mathrm{N}^{2} \mathrm{LO}-\Delta_{c}$ current to reproduce, for a given cutoff $\Lambda$ and Hamiltonian model, the experimental isovector magnetic moment. The resulting values for the parameter $f^{\prime}$, defined in Eq. (7.1), are listed in TableVII. The violent change of $f^{\prime}$ as the cutoff $\Lambda$ is increased is due to the fact that the short-range behavior of the $\mathrm{N}^{2} \mathrm{LO}-\Delta_{c}$ current is governed by a Gaussian of half-width $2 / \Lambda$.

\begin{tabular}{c|c|c}
\hline \hline$\Lambda(\mathrm{MeV})$ & AV18/UIX & CDB/UIX \\
\hline 500 & -3.036 & -38.57 \\
600 & +11.86 & -25.25 \\
800 & +51.13 & +3.485 \\
\hline \hline
\end{tabular}

TABLE VII: The parameter $f^{\prime}$ required to reproduce, for a given value of the cutoff $\Lambda$, the experimental isovector combination of the trinucleon magnetic moments with the AV18/UIX or CDB/UIX* Hamiltonian models.

However, with the values of the parameters $f$ and $f^{\prime}$ fixed as discussed above, the current up to $\mathrm{N}^{2} \mathrm{LO}$ is now completely determined. We can therefore use it to make predictions for the cross section $\sigma_{T}$ and photon circular polarization parameter $R_{c}$ measured in the reaction ${ }^{2} \mathrm{H}(n, \gamma)^{3} \mathrm{H}$. At thermal energies this process proceeds through S-wave capture predominantly via magnetic dipole transitions from the initial doublet $J=1 / 2$ and quartet $J=3 / 2 n$ - $d$ scattering states. In addition, there is a small contribution due to an electric quadrupole transition from the initial quartet state. We adopt here the notation and conventions of Ref. [29] and define

$$
m_{22}=\widetilde{M}_{1}^{01 / 21 / 2}, \quad m_{44}=\widetilde{M}_{1}^{03 / 23 / 2}, \quad e_{44}=\widetilde{E}_{2}^{03 / 23 / 2}
$$

where $\widetilde{M}_{\ell}^{L S J}$ and $\widetilde{E}_{\ell}^{L S J}$ are the reduced matrix elements (RME's) of the magnetic and electric multipole operators of order $\ell$, normalized as in Eq. (6.3) of Ref. [29]. In terms of these 
RME's, the capture total cross section is given by

$$
\sigma_{T}=\frac{2}{9} \frac{\alpha}{v_{\mathrm{rel}}} \frac{q^{3}}{4 m_{N}^{2}}\left(\left|m_{22}\right|^{2}+\left|m_{44}\right|^{2}+\left|e_{44}\right|^{2}\right)
$$

where $\alpha=e^{2} /(4 \pi)$ is the fine structure constant, $v_{\text {rel }}$ is the $d$ - $n$ relative velocity, $q$ is the energy of the emitted $\gamma$ ray, and $m_{N}$ is the nucleon mass. Similarly, the circular polarization $P_{\Gamma}$ resulting from $\mathrm{S}$-wave capture of a neutron polarized along the direction $\mathbf{P}_{n}$ is proportional to the parameter $R_{c}[29]$, i.e. $P_{\Gamma}=R_{c} \mathbf{P}_{n} \cdot \hat{\mathbf{q}}$, where

$$
R_{c}=-\frac{1}{3}\left[1-\frac{(7 / 2)\left|m_{44}\right|^{2}+\sqrt{8} \operatorname{Re}\left(m_{22} m_{44}^{*}\right)+(5 / 2)\left|e_{44}\right|^{2}+\sqrt{24} \operatorname{Im}\left(m_{22} e_{44}^{*}\right)-\sqrt{3} \operatorname{Im}\left(m_{44} e_{44}^{*}\right)}{\left|m_{22}\right|^{2}+\left|m_{44}\right|^{2}+\left|e_{44}\right|^{2}}\right]
$$

The predicted RME's are listed in TableVIII, the cross section and parameter $R_{c}$ in Table IX. Note that only results corresponding to the AV18/UIX Hamiltonian model are available at this time for the reason explained earlier. At LO the quartet $m_{44}$ is, in absolute value,

\begin{tabular}{c|c|c|c||c|c|c||c|c|c} 
& \multicolumn{4}{|c}{$m_{22}$} & \multicolumn{1}{c}{$m_{44}$} & \multicolumn{3}{c}{$e_{44}$} \\
\hline \hline$\Lambda(\mathrm{MeV})$ & 500 & 600 & 800 & 500 & 600 & 800 & 500 & 600 & 800 \\
\hline $\mathrm{LO}$ & -10.6 & -10.6 & -10.6 & 13.5 & 13.5 & 13.5 & -0.14 & -0.14 & -0.14 \\
$\mathrm{LO}+\mathrm{NLO}$ & -13.2 & -12.5 & -11.5 & 13.3 & 13.4 & 13.5 & +0.02 & +0.02 & +0.02 \\
$\mathrm{LO}+\cdots+\mathrm{N}^{2} \mathrm{LO}-\mathrm{RC}$ & -12.0 & -11.3 & -10.3 & 13.5 & 13.6 & 13.7 & +0.02 & +0.02 & +0.02 \\
$\mathrm{LO}+\cdots+\mathrm{N}^{2} \mathrm{LO}-\Delta$ & -20.2 & -19.4 & -18.4 & 12.6 & 12.7 & 12.8 & +0.02 & +0.02 & +0.02 \\
$\mathrm{LO}+\cdots+\mathrm{N}^{2} \mathrm{LO}-\Delta_{c}$ & -20.6 & -18.3 & -15.6 & 12.6 & 12.6 & 12.7 & +0.02 & +0.02 & +0.02 \\
\hline \hline
\end{tabular}

TABLE VIII: Cumulative contributions (in $\mathrm{fm}^{3 / 2}$ ) to the reduced matrix elements (RME's) of the ${ }^{2} \mathrm{H}(n, \gamma){ }^{3} \mathrm{H}$ reaction at thermal energies, obtained with the AV18/UIX Hamiltonian model and cutoff values in the range 500-800 MeV. See text for notation. The $m_{22}$ and $m_{44}$ RME's are purely imaginary, while the $e_{44}$ RME is purely real.

about $27 \%$ larger than the doublet $m_{22}$. However, the contributions at NLO and $\mathrm{N}^{2} \mathrm{LO}$ are large and interfere constructively with those at LO for $m_{22}$, while they are much smaller and interfere destructively for $m_{44}$. Consequently, the doublet $m_{22}$ at $\mathrm{N}^{2} \mathrm{LO}$ is found to be larger than the quartet $m_{44}$ by $63 \%-23 \%$ as the cutoff $\Lambda$ is increased from $500 \mathrm{MeV}$ to 800 $\mathrm{MeV}$. The calculation of the RME's is carried out with the Monte Carlo (MC) integration techniques of Ref. [29], and the results reported in Table VIII] are obtained from a random walk consisting of a large number (of the order of $2 \mathrm{M}$ ) configurations. The statistical errors associated with these MC integrations are typically less than $2 \%$ for $m_{22}$ and much less than $1 \%$ for $m_{44}$. However, they are $25 \%$ for $e_{44}$ at LO, and indeed much larger than the central value at $\mathrm{N}^{2} \mathrm{LO}$, so that beyond $\mathrm{LO}$ the value of this RME is consistent with zero. We note that in S-wave capture the $e_{44}$ RME is predominantly due to transitions $\mathrm{S}\left({ }^{2} \mathrm{H}\right) \rightarrow \mathrm{D}\left({ }^{3} \mathrm{H}\right)$ and $\mathrm{D}\left({ }^{2} \mathrm{H}\right) \rightarrow \mathrm{S}\left({ }^{3} \mathrm{H}\right)$, where $\mathrm{S}$ and $\mathrm{D}$ denote $\mathrm{S}$ - and D-wave components in the ${ }^{2} \mathrm{H}$ and ${ }^{3} \mathrm{H}$ ground states. In the case of the AV18/UIX Hamiltonian, the contributions associated with these transitions interfere destructively, thus producing a small $e_{44}$. This cancellation was found to be significantly model dependent in Ref. [29], and use of CDB/UIX* wave functions would presumably produce somewhat different results for this RME in view of the considerably weaker tensor force of CDB relative to AV18 at intermediate and short range. 


\begin{tabular}{c|c|c|c||c|c|c} 
& \multicolumn{3}{|c}{$\sigma_{T}$} & \multicolumn{3}{c}{$R_{c}$} \\
\hline \hline$\Lambda(\mathrm{MeV})$ & 500 & 600 & 800 & 500 & 600 & 800 \\
\hline $\mathrm{LO}$ & 0.229 & 0.229 & 0.229 & -0.060 & -0.060 & -0.060 \\
$\mathrm{LO}+\mathrm{NLO}$ & 0.272 & 0.260 & 0.243 & -0.218 & -0.182 & -0.123 \\
$\mathrm{LO}+\cdots+\mathrm{N}^{2} \mathrm{LO}-\mathrm{RC}$ & 0.252 & 0.241 & 0.226 & -0.152 & -0.109 & -0.041 \\
$\mathrm{LO}+\cdots+\mathrm{N}^{2} \mathrm{LO}-\Delta$ & 0.438 & 0.416 & 0.389 & -0.432 & -0.418 & -0.397 \\
$\mathrm{LO}+\cdots+\mathrm{N}^{2} \mathrm{LO}-\Delta_{c}$ & 0.450 & 0.382 & 0.315 & -0.437 & -0.398 & -0.331 \\
\hline \hline
\end{tabular}

TABLE IX: Cumulative contributions to the cross section $\sigma_{T}$ (in mb) and photon polarization parameter $R_{c}$ of the reaction ${ }^{2} \mathrm{H}(n, \gamma){ }^{3} \mathrm{H}$ at thermal energies, obtained with the AV18/UIX Hamiltonian model and cutoff values in the range 500-800 MeV. The experimental values for $\sigma_{T}$ and $R_{c}$ are respectively $(0.508 \pm 0.015) \mathrm{mb}$ from Ref. [6] and $-0.42 \pm 0.03$ from Ref. [30].

At $\mathrm{N}^{2} \mathrm{LO}$ the cross section is underpredicted by theory by (11-38)\% as the cutoff is increased from $500 \mathrm{MeV}$ to $800 \mathrm{MeV}$. This rather drastic cutoff dependence is mostly due to the contribution of the $\mathrm{N}^{2} \mathrm{LO}-\Delta_{c}$ current. Indeed removing it leads to a much weaker variation of the cross section-roughly $\pm 5 \%$ about the value obtained with $\Lambda=600 \mathrm{MeV}$ (next to last row of Table IX). It will be interesting to see to what extent, if any, loop corrections at $\mathrm{N}^{3} \mathrm{LO}$ will improve the present predictions, and in particular reduce the cutoff dependence.

The photon polarization parameter is very sensitive to contributions of NLO and $\mathrm{N}^{2} \mathrm{LO}$ currents, which produce more than a sixfold increase, in absolute value, of the LO result, and bring it into much closer agreement with the measured value. All results listed in Table IX for $R_{c}$ (and $\sigma_{T}$ ) include the small $e_{44}$ RME, although it only has a significant effect for the LO prediction $\left(R_{c}=-0.060\right.$ versus -0.072 depending on whether $e_{44}$ is retained or not). The cause of the $R_{c}$ sensitivity to corrections beyond LO becomes clear by examining the expression given in Eq. (7.10). Neglecting $e_{44}$, it reads

$$
1+3 R_{c}=\frac{7 / 2-\sqrt{8}\left|m_{22} / m_{44}\right|}{1+\left|m_{22} / m_{44}\right|^{2}},
$$

and therefore the value attained by $R_{c}$ is driven by the ratio $\left|m_{22} / m_{44}\right|$, which is about 0.79 at LO and ranges from 1.63 to 1.23 for $\Lambda=500-800 \mathrm{MeV}$.

We conclude this section by remarking that recent calculations of $n$ - $d$ capture observables [31], based on an effective field theory formulated in terms of nucleon, deuteron, and triton fields with gradient couplings, seem to lead to predictions which are in agreement with data. However, it should be stressed that such a theory cannot be applied to other processes, for example the $n^{-}{ }^{3} \mathrm{He}$ capture, without including additional degrees of freedom. It is in this sense of a more limited scope than the approach advocated in the present work.

\section{CONCLUSIONS AND OUTLOOK}

The goals of the present work were twofold: firstly, to derive the nuclear electromagnetic current up to one loop, i.e. up to $\mathrm{N}^{3} \mathrm{LO}$, within an effective-field-theory approach including explicit nucleons, $\Delta$-isobars, and pions, and secondly to implement this formalism in the calculation of a number of few-nucleon electromagnetic observables at low energy by using 
accurate wave functions - the so-called hybrid approach, advocated, for example, in Refs. [4, 16]. This last objective has been partially carried out here, since results have been reported only at $\mathrm{N}^{2} \mathrm{LO}$, i.e. ignoring loop corrections.

Up to this order, the only isoscalar terms are those generated in a non-relativistic expansion of the one-body current: they provide a (cutoff-independent) $1 \%$ correction-relative to $\mathrm{LO}$ - to the deuteron and isoscalar combination of the trinucleon magnetic moments. This correction is of opposite sign to the LO contribution, and therefore increases the underprediction of the corresponding experimental values from $(0.9 \pm 0.3) \%$ for the deuteron and $(2.7 \pm 0.9) \%$ for the trinucleons at LO to, respectively, $(1.9 \pm 0.3) \%$ and $(3.8 \pm 0.8) \%$ at $\mathrm{N}^{2} \mathrm{LO}$. The spread reflects differences in the short-range behavior of the AV18 and CDB potentials, in particular the weaker tensor components of the latter relative to the former in this range.

At NLO, isovector terms arise from the pion seagull and in-flight contributions, while at $\mathrm{N}^{2} \mathrm{LO}$, in addition to the relativistic corrections mentioned above, isovector terms due to $\Delta$-isobar excitation are also obtained. The value for the combination of coupling constants $\left(g_{A} / F_{\pi}\right)^{2}$ entering the NLO two-body currents is that inferred by an analysis of nucleonnucleon elastic scattering data [26]. However, the strengths of the $\mathrm{N}^{2} \mathrm{LO}$ two-body $\Delta$ excitation currents, i.e. the combinations $\mu^{*} g_{A} h_{A} / F_{\pi}^{2}$ and $\mu^{*} D_{T}$ in Eqs. (4.12) and (4.13), respectively, have been determined, as functions of the cutoff $\Lambda$ and for the Hamiltonian model of interest, by reproducing the cross section for $n-p$ capture and the isovector combination of the trinucleon magnetic moments. This current has then been used to make predictions - with the AV18/UIX model only - for the cross section $\sigma_{T}$ and photon circular polarization parameter $R_{c}$. The experimental $\sigma_{T}\left(\left|R_{c}\right|\right)$ is found to be underestimated by $11 \%$ (overestimated by $4 \%$ ) for $\Lambda=500 \mathrm{MeV}$ and $38 \%$ (underestimated by $21 \%$ ) for $\Lambda=800$ $\mathrm{MeV}$. We note that the parameter $R_{c}$ is mostly sensitive to the ratio of doublet to quartet magnetic dipole transition matrix elements $\left|m_{22} / m_{44}\right|$ (the cross section is proportional to $\left.\left|m_{22}\right|^{2}+\left|m_{44}\right|^{2}\right)$.

The results display a significant cutoff dependence, particularly so for the $\mathrm{N}^{2} \mathrm{LO}$ contributions associated with $\Delta$ isobar degrees of freedom. Indeed these contributions are much larger than those at NLO. This is partly due to the fact that the two NLO (pion seagull and in-flight) terms interfere destructively. For example, the seagull (in-flight) contributions to $m_{22}$ and $m_{44}$, in units of $\mathrm{fm}^{3 / 2}$ and for $\Lambda=500 \mathrm{MeV}$, are respectively $-9.1 i(+6.5 i)$ and $-0.8 i(+0.6 i)$, which add up to the NLO values $-2.6 i$ and $-0.2 i$ from Table VIII, As a result $\sigma_{T}=0.425 \mathrm{mb}$ and $R_{c}=-0.425$ at $\mathrm{LO}+\mathrm{NLO}$ (seagull only), which should be compared to $\sigma_{T}=0.272 \mathrm{mb}$ and $R_{c}=-0.218$ at LO+NLO (seagull+in-flight) from the second row of Table IX.

The relatively large $\Delta$-excitation contributions also point to the need for including loop corrections at $\mathrm{N}^{3} \mathrm{LO}$, which these $\mathrm{N}^{2} \mathrm{LO}$ currents, because of the procedure adopted here to determine their strength, are implicitly making up for. This is also evident by examining the results for the $n-p$ capture cross section in Table IV. Had we chosen to fix the $N-\Delta$ transition axial coupling constant $h_{A}$ and magnetic moment $\mu^{*}$ via, respectively, the $\Delta$ width and $\gamma-N$ data at resonance, the contribution of the $\mathrm{N}^{2} \mathrm{LO}-\Delta$ current would have been considerably smaller than that at NLO, and would have fallen more in line with naive expectations.

The $\mathrm{N}^{3} \mathrm{LO}$ corrections will presumably reduce the cutoff dependence in the $n$ - $d$ predictions, and hopefully bring theory into more satisfying agreement with experiment. For the time being, we only observe that calculations [9] based on the AV18/UIX Hamiltonian with leading two- and three-body currents constructed consistently - in the sense of being 
exactly conserved - with, respectively, the AV18 two-nucleon and UIX three-nucleon potentials overestimated both $\sigma_{T}$ and $\left|R_{c}\right|$ by about $10 \%$ in the ${ }^{2} \mathrm{H}(n, \gamma)^{3} \mathrm{H}$ process at thermal energies, while at the same time providing an excellent description of cross section data for the $p$ - $d$ radiative capture in the energy range from a few to $80 \mathrm{keV}$, and, in particular, of the astrophysical factor at zero energy extrapolated from these data.

Thus, as already emphasized in the Introduction, very low-energy radiative (and weak) capture reactions involving three- and four-body nuclei constitute a crucial testing ground for the models used to describe the ground- and scattering-state wave functions - and indirectly, the underlying interactions which generate these wave functions - and the many-body electroweak current operators [8].

The next stage in the research program we have undertaken is to incorporate the $\mathrm{N}^{3} \mathrm{LO}$ operators derived here into the calculations of the captures and magnetic moments involving light nuclei (with mass number $A \leq 8$ ), and indeed to extend these calculations to also include $p$ - $d$ capture at energies up to a few MeV's, and possibly four-nucleon processes, in particular ${ }^{3} \mathrm{He}(n, \gamma){ }^{4} \mathrm{He}$ at thermal energies. Of course, at $\mathrm{N}^{3} \mathrm{LO}$ three-body currents also occur, and will need to be derived. Work along these lines is being pursued vigorously.

\section{Acknowledgments}

We would like to thank L. Girlanda, A. Kievsky, L.E. Marcucci, and M. Viviani for discussions and for letting us use their trinucleon wave functions, E. Epelbaum and S. Kölling for an interesting conversation and for sharing with us some of their results on the same topic before publication, and V. Cirigliano for discussions in the early phase of this work. One of the authors (R.S.) would also like to thank the Physics Department of the University of Pisa, the INFN Pisa branch, and especially the Pisa group for the support and warm hospitality extended to him on several occasions.

The work R.S. is supported by the U.S. Department of Energy, Office of Nuclear Physics, under contract DE-AC05-06OR23177, and that of J.L.G. by the National Science Foundation under grant PHY-0555559. The calculations were made possible by grants of computing time from the National Energy Research Supercomputer Center.

\section{APPENDIX A: VERTICES}

The interaction Hamiltonians in Secs. $\llbracket \mathrm{A}$ and $\Pi \mathrm{IB}$ are assumed to be normal-ordered. Explicit expressions for the associated vertices are easily derived (these expressions include the $1 / \sqrt{2 \omega_{k_{i}}}$ factors from pion fields) :

i) Strong-interaction vertices:

$$
\begin{aligned}
\left\langle\mathbf{p}^{\prime}, \chi^{\prime} ; \mathbf{k}, a\left|H_{\pi N N}\right| \mathbf{p}, \chi\right\rangle & =-i \frac{g_{A}}{F_{\pi}} \frac{\boldsymbol{\sigma} \cdot \mathbf{k}}{\sqrt{2 \omega_{k}}} \tau_{a}, \\
\left\langle\mathbf{p}_{\Delta}^{\prime}, \chi_{\Delta}^{\prime} ; \mathbf{k}, a\left|H_{\pi N \Delta}\right| \mathbf{p}, \chi\right\rangle & =-i \frac{h_{A}}{F_{\pi}} \frac{\mathbf{S} \cdot \mathbf{k}}{{\sqrt{2 \omega_{k}}}_{a}} T_{a} \\
\left\langle\mathbf{p}^{\prime}, \chi^{\prime} ; \mathbf{k}_{1}, a ; \mathbf{k}_{2}, b\left|H_{\pi \pi N N}\right| \mathbf{p}, \chi\right\rangle & =-\frac{i}{F_{\pi}^{2}} \frac{\omega_{k_{1}}-\omega_{k_{2}}}{\sqrt{4 \omega_{k_{1}} \omega_{k_{2}}}} \epsilon_{a b c} \tau_{c}
\end{aligned}
$$




$$
\begin{aligned}
&\left\langle\mathbf{p}_{1}^{\prime}, \chi_{1}^{\prime} ; \mathbf{p}_{2}^{\prime}, \chi_{2}^{\prime}\left|H_{\mathrm{CT}, 1}\right| \mathbf{p}_{1}, \chi_{1} ; \mathbf{p}_{2}, \chi_{2}\right\rangle=\sum_{\alpha=S, T} C_{\alpha} \boldsymbol{\Gamma}_{1 \alpha} \cdot \boldsymbol{\Gamma}_{2 \alpha}, \\
&\left\langle\mathbf{p}^{\prime}, \chi^{\prime} ; \mathbf{p}_{\Delta}^{\prime}, \chi_{\Delta}^{\prime}\left|H_{\mathrm{CT}, 2}\right| \mathbf{p}_{1}, \chi_{1} ; \mathbf{p}_{2}, \chi_{2}\right\rangle=D_{T} \boldsymbol{\tau}_{1} \cdot \mathbf{T}_{2} \boldsymbol{\sigma}_{1} \cdot \mathbf{S}_{2}, \\
&\left\langle\mathbf{p}^{\prime}, \chi^{\prime} ; \mathbf{p}_{\Delta}^{\prime}, \chi_{\Delta}^{\prime}\left|H_{\mathrm{CT}, 3}\right| \mathbf{p}, \chi ; \mathbf{p}_{\Delta}, \chi_{\Delta}\right\rangle=\sum_{\alpha=S, T} C_{\alpha}^{\prime} \boldsymbol{\Gamma}_{1 \alpha} \cdot \boldsymbol{\Gamma}_{2 \alpha}^{\prime}, \\
&\left\langle\mathbf{p}_{1, \Delta}^{\prime}, \chi_{1, \Delta}^{\prime} ; \mathbf{p}_{2, \Delta}^{\prime}, \chi_{2, \Delta}^{\prime}\left|H_{\mathrm{CT}, 4}\right| \mathbf{p}_{1}, \chi_{1} ; \mathbf{p}_{2}, \chi_{2}\right\rangle=D_{T}^{\prime} \mathbf{T}_{1} \cdot \mathbf{T}_{2} \mathbf{S}_{1} \cdot \mathbf{S}_{2}, \\
&\left\langle\mathbf{p}_{\Delta}^{\prime}, \chi_{\Delta}^{\prime} ; \mathbf{p}^{\prime}, \chi^{\prime}\left|H_{\mathrm{CT}, 5}\right| \mathbf{p}, \chi ; \mathbf{p}_{\Delta}, \chi_{\Delta}\right\rangle=D_{T}^{\prime \prime} \mathbf{T}_{1} \cdot \mathbf{T}_{2}^{\dagger} \mathbf{S}_{1} \cdot \mathbf{S}_{2}^{\dagger}, \\
&\left\langle\mathbf{p}^{\prime}, \chi^{\prime} ; \mathbf{k}_{1}, a ; \mathbf{k}_{2}, b ; \mathbf{k}_{3}, c\left|H_{3 \pi}\right| \mathbf{p}, \chi\right\rangle=\frac{2 i g_{A}}{F_{\pi}^{3}} \frac{1}{\sqrt{8 \omega_{k_{1}} \omega_{k_{2}} \omega_{k_{3}}}}\left(\boldsymbol{\sigma}_{\mathbf{k}_{1}} \tau_{a} \delta_{b c}\right. \\
&\left.+\boldsymbol{\sigma} \cdot \mathbf{k}_{2} \tau_{b} \delta_{c a}+\boldsymbol{\sigma} \cdot \mathbf{k}_{3} \tau_{c} \delta_{a b}\right),
\end{aligned}
$$

$$
\begin{aligned}
\left\langle\mathbf{k}_{1}, a ; \mathbf{k}_{2}, b ; \mathbf{k}_{3}, c ; \mathbf{k}_{4}, d\left|H_{4 \pi}\right| 0\right\rangle & =-\frac{4}{F_{\pi}^{2}} \frac{1}{\sqrt{16 \omega_{k_{1}} \omega_{k_{2}} \omega_{k_{3}} \omega_{k_{4}}}}\left[\delta_{a b} \delta_{c d}\left(k_{1 \mu} k_{2}^{\mu}+k_{3 \mu} k_{4}^{\mu}+m_{\pi}^{2}\right)\right. \\
& +\delta_{a c} \delta_{b d}\left(k_{1 \mu} k_{3}^{\mu}+k_{2 \mu} k_{4}^{\mu}+m_{\pi}^{2}\right) \\
& \left.+\delta_{a d} \delta_{b c}\left(k_{1 \mu} k_{4}^{\mu}+k_{2 \mu} k_{3}^{\mu}+m_{\pi}^{2}\right)\right]
\end{aligned}
$$

ii) Electromagnetic-interaction vertices:

$$
\begin{aligned}
& \left\langle\mathbf{p}^{\prime}, \chi^{\prime} ; \mathbf{k}, a\left|H_{\gamma \pi N N}\right| \mathbf{p}, \chi ; \mathbf{q}, \lambda\right\rangle=e \frac{g_{A}}{F_{\pi}} \frac{\boldsymbol{\sigma}}{\sqrt{2 \omega}_{k}} \cdot \frac{\hat{\mathbf{e}}_{\mathbf{q} \lambda}}{{\sqrt{2 \omega_{q}}}_{z a b} \tau_{b}}, \\
& \left\langle\mathbf{p}^{\prime}, \chi_{\Delta}^{\prime} ; \mathbf{k}, a\left|H_{\gamma \pi N \Delta}\right| \mathbf{p}, \chi ; \mathbf{q}, \lambda\right\rangle=e \frac{h_{A}}{F_{\pi}} \frac{\mathbf{S}}{\sqrt{2 \omega}_{k}} \cdot \frac{\hat{\mathbf{e}}_{\mathbf{q} \lambda}}{\sqrt{2 \omega}_{q}} \epsilon_{z a b} T_{b}, \\
& \left\langle\mathbf{k}_{1}, a ; \mathbf{k}_{2}, b\left|H_{\gamma \pi \pi}\right| \mathbf{q}, \lambda\right\rangle=i e \frac{\mathbf{k}_{1}-\mathbf{k}_{2}}{\sqrt{4 \omega_{k_{1}} \omega_{k_{2}}}} \cdot \frac{\hat{\mathbf{e}}_{\mathbf{q} \lambda}}{\sqrt{2 \omega_{q}}} \epsilon_{z a b}, \\
& \left\langle\mathbf{p}^{\prime}, \chi^{\prime} ; \mathbf{k}_{1}, a ; \mathbf{k}_{2}, b\left|H_{\gamma \pi \pi N N}\right| \mathbf{p}, \chi ; \mathbf{q}, \lambda\right\rangle=-\frac{e}{F_{\pi}^{2}} \frac{1}{\sqrt{4 \omega_{k_{1}} \omega_{k_{2}}}} \\
& \frac{\hat{\mathbf{e}}_{\mathbf{q} \lambda}}{\sqrt{2 \omega_{q}}} \cdot \frac{\left(\mathbf{p}^{\prime}+\mathbf{p}\right)+i \boldsymbol{\sigma} \times\left(\mathbf{p}^{\prime}-\mathbf{p}\right)}{2 m_{N}}\left(\delta_{a z} \tau_{b}+\delta_{b z} \tau_{a}-2 \delta_{a b} \tau_{z}\right), \\
& \left\langle\mathbf{p}^{\prime}, \chi^{\prime} ; \mathbf{k}_{1}, a ; \mathbf{k}_{2}, b ; \mathbf{k}_{3}, c\left|H_{\gamma 3 \pi}\right| \mathbf{p}, \chi ; \mathbf{q}, \lambda\right\rangle=-2 e \frac{g_{A}}{F_{\pi}^{3}} \frac{\boldsymbol{\sigma}}{\sqrt{8 \omega_{k_{1}} \omega_{k_{2}} \omega_{k_{3}}}} \cdot \frac{\hat{\mathbf{e}}_{\mathbf{q} \lambda}}{\sqrt{2 \omega_{q}}} \\
& \tau_{d}\left(\epsilon_{z a d} \delta_{b c}+\epsilon_{z b d} \delta_{c a}+\epsilon_{z c d} \delta_{a b}\right) \text {, } \\
& \left\langle\mathbf{k}_{1}, a ; \mathbf{k}_{2}, b ; \mathbf{k}_{3}, c ; \mathbf{k}_{4}, d\left|H_{\gamma 4 \pi}\right| \mathbf{q}, \lambda\right\rangle=-i e \frac{4}{F_{\pi}^{2}} \frac{1}{\sqrt{16 \omega_{k_{1}} \omega_{k_{2}} \omega_{k_{3}} \omega_{k_{4}}}} \frac{\hat{\mathbf{e}}_{\mathbf{q} \lambda}}{\sqrt{2 \omega_{q}}} \\
& {\left[\delta_{c d} \epsilon_{z a b}\left(\mathbf{k}_{1}-\mathbf{k}_{2}\right)+\delta_{a b} \epsilon_{z c d}\left(\mathbf{k}_{3}-\mathbf{k}_{4}\right)\right.} \\
& +\delta_{b d} \epsilon_{z a c}\left(\mathbf{k}_{1}-\mathbf{k}_{3}\right)+\delta_{a d} \epsilon_{z b c}\left(\mathbf{k}_{2}-\mathbf{k}_{3}\right) \\
& \left.+\delta_{a c} \epsilon_{z b d}\left(\mathbf{k}_{2}-\mathbf{k}_{4}\right)+\delta_{b c} \epsilon_{z a d}\left(\mathbf{k}_{1}-\mathbf{k}_{4}\right)\right] \text {, }
\end{aligned}
$$




$$
\begin{aligned}
\left\langle\mathbf{p}^{\prime}, \chi^{\prime}\left|H_{\gamma N N}\right| \mathbf{p}, \chi ; \mathbf{q}, \lambda\right\rangle & =-\frac{e}{2 m_{N}} \frac{\hat{\mathbf{e}}_{\mathbf{q} \lambda}}{\sqrt{2 \omega_{q}}} \cdot\left[e_{N}\left(\mathbf{p}^{\prime}+\mathbf{p}\right)+i \mu_{n} \boldsymbol{\sigma} \times \mathbf{q}\right], \\
\left\langle\mathbf{p}_{\Delta}^{\prime}, \chi_{\Delta}^{\prime}\left|H_{\gamma N \Delta}\right| \mathbf{p}, \chi ; \mathbf{q}, \lambda\right\rangle & =-i \frac{e \mu^{*}}{2 m_{N}} \frac{\hat{\mathbf{e}}_{\mathbf{q} \lambda}}{\sqrt{2 \omega_{q}}} \cdot \mathbf{S} \times \mathbf{q} T_{z} .
\end{aligned}
$$

In these expressions $\mathbf{p}$ and $\mathbf{p}_{\Delta}$ denote nucleon and $\Delta$-isobar momenta in spin-isospin states specified by $\chi$ and $\chi_{\Delta}$ respectively, while the k's and $a, b, \ldots$ denote pion momenta in isospin states $a, b, \ldots$, and $\mathbf{q}$ and $\lambda$ the photon momentum and polarization state. For brevity, on the r.h.s. of the equations above the spin-isospin states of the nucleon and $\Delta$ isobar as well as the $\delta$-functions enforcing three-momentum conservation, are not shown explicitly. In

Eq. (A10), the notation $k_{i}^{\mu} k_{j \mu}$ denotes the combination $\omega_{k_{i}} \omega_{k_{j}}-\mathbf{k}_{i} \cdot \mathbf{k}_{j}$. Finally, vertices involving $\Delta$-isobar deexcitation into a nucleon are obtained by replacing $\mathbf{S}$ and $\mathbf{T}$ by their adjoint operators $\mathbf{S}^{\dagger}$ and $\mathbf{T}^{\dagger}$, while vertices in which one or more pions are in the initial state are obtained from those listed in Eqs. (A1)-(A3), (A9), (A10), (A13), and (A16) by replacing $\mathbf{k}_{i} \rightarrow-\mathbf{k}_{i}$ and/or $\omega_{k_{i}} \rightarrow-\omega_{k_{i}}$ (of course, the energy replacements are not to be carried out in the pion-field normalization factors). For example,

$$
\left\langle\mathbf{p}^{\prime}, \chi^{\prime} ; \mathbf{k}_{1}, a\left|H_{\pi \pi N N}\right| \mathbf{p}, \chi ; \mathbf{k}_{2}, b\right\rangle=-\frac{i}{F_{\pi}^{2}} \frac{\omega_{k_{1}}+\omega_{k_{2}}}{\sqrt{4 \omega_{k_{1}} \omega_{k_{2}}}} \epsilon_{a b c} \tau_{c} .
$$

\section{APPENDIX B: CONFIGURATION-SPACE REPRESENTATION}

We list here the configuration-space representation of the two-body currents at NLO and $\mathrm{N}^{2} \mathrm{LO}$. To this end, it is convenient to define $z_{\pi} \equiv m_{\pi} r, z_{\Lambda} \equiv \Lambda r, z_{L} \equiv r L(q ; x)$,

$$
z_{ \pm} \equiv m_{\pi} / \Lambda \pm z_{\Lambda} / 2, \quad z_{ \pm}^{*} \equiv L(q ; x) / \Lambda \pm z_{\Lambda} / 2
$$

and the complement error function

$$
\phi(z) \equiv \frac{2}{\sqrt{\pi}} \int_{z}^{\infty} \mathrm{d} t \mathrm{e}^{-t^{2}}
$$

The dependence of $z_{L}$ and $z_{ \pm}^{*}$ upon the variable $x$ is understood. Then the functions $f_{\Lambda}(r)$, Eq. (4.8), along with its first derivative $f_{\Lambda}^{\prime}(r)$, and $\nabla g_{\Lambda}(\mathbf{r}, \mathbf{q})$, Eq. (4.9), are given by

$$
\begin{gathered}
f_{\Lambda}(r)=\frac{m_{\pi}}{8 \pi} \frac{\mathrm{e}^{m_{\pi}^{2} / \Lambda^{2}}}{z_{\pi}}\left[\phi\left(z_{-}\right) \mathrm{e}^{-z_{\pi}}-\phi\left(z_{+}\right) \mathrm{e}^{z_{\pi}}\right] \\
f_{\Lambda}^{\prime}(r)=\frac{m_{\pi}^{2}}{8 \pi} \frac{\mathrm{e}^{m_{\pi}^{2} / \Lambda^{2}}}{z_{\pi}^{2}}\left[\phi\left(z_{+}\right) \mathrm{e}^{z_{\pi}}\left(1-z_{\pi}\right)-\phi\left(z_{-}\right) \mathrm{e}^{-z_{\pi}}\left(1+z_{\pi}\right)\right]+\frac{\Lambda^{2}}{4 \pi \sqrt{\pi}} \frac{\mathrm{e}^{-z_{\Lambda}^{2} / 4}}{z_{\Lambda}}, \\
\left.\nabla g_{\Lambda}(\mathbf{r}, \mathbf{q})\right|_{\perp}=\hat{\mathbf{r}} \int_{-1}^{+1} \mathrm{~d} x \mathrm{e}^{-i x \mathbf{q} \cdot \mathbf{r} / 2} E_{q}(x, r)
\end{gathered}
$$

where only the transverse part of $\nabla g_{\Lambda}(\mathbf{r}, \mathbf{q})$ (orthogonal to the photon momentum $\mathbf{q}$ ) is of interest, and

$$
E_{q}(x, r)=\frac{\mathrm{e}^{L^{2}(q ; x) / \Lambda^{2}}}{8 \pi z_{\Lambda}^{2}}\left[\phi\left(z_{+}^{*}\right) \mathrm{e}^{z_{L}}\left(1-z_{L}-z_{\Lambda}^{2} / 2\right)-\phi\left(z_{-}^{*}\right) \mathrm{e}^{-z_{L}}\left(1+z_{L}-z_{\Lambda}^{2} / 2\right)\right]+\frac{\mathrm{e}^{-z_{\Lambda}^{2} / 4}}{4 \pi \sqrt{\pi} z_{\Lambda}} .
$$


In the limit $\Lambda \rightarrow \infty$ these functions reduce to:

$$
f_{\infty}(r)=\frac{m_{\pi}}{4 \pi} \frac{\mathrm{e}^{-z_{\pi}}}{z_{\pi}}, \quad f_{\infty}^{\prime}(r)=-\frac{m_{\pi}^{2}}{4 \pi} \frac{\mathrm{e}^{-z_{\pi}}}{z_{\pi}^{2}}\left(1+z_{\pi}\right), \quad E_{q, \infty}(r)=\frac{\mathrm{e}^{-z_{L}}}{8 \pi} .
$$

The complete NLO current - the sum of the two contributions in Eqs. (4.6) and (4.7) - is then written as

$$
\begin{aligned}
\left.\mathbf{j}^{\mathrm{NLO}}(\mathbf{q})\right|_{\perp} & =e \frac{g_{A}^{2}}{F_{\pi}^{2}}\left(\boldsymbol{\tau}_{1} \times \boldsymbol{\tau}_{2}\right)_{z}\left\{e^{i \mathbf{q} \cdot \mathbf{r}_{1}} f_{\Lambda}^{\prime}(r) \boldsymbol{\sigma}_{1}\left(\boldsymbol{\sigma}_{2} \cdot \hat{\mathbf{r}}\right)+\mathrm{e}^{i \mathbf{q} \cdot \mathbf{r}_{2}} f_{\Lambda}^{\prime}(r) \boldsymbol{\sigma}_{2}\left(\boldsymbol{\sigma}_{1} \cdot \hat{\mathbf{r}}\right)\right. \\
& +\mathrm{e}^{i \mathbf{q} \cdot \mathbf{R}}\left[\frac{g_{\Lambda}^{(1)}(\mathbf{r}, \mathbf{q})}{r^{2}}\left[\boldsymbol{\sigma}_{1}\left(\boldsymbol{\sigma}_{2} \cdot \hat{\mathbf{r}}\right)+\boldsymbol{\sigma}_{2}\left(\boldsymbol{\sigma}_{1} \cdot \hat{\mathbf{r}}\right)+\hat{\mathbf{r}}\left(\boldsymbol{\sigma}_{1} \cdot \boldsymbol{\sigma}_{2}\right)\right]+i \frac{g_{\Lambda}^{(2)}(\mathbf{r}, \mathbf{q})}{r} \boldsymbol{\sigma}_{1}\left(\boldsymbol{\sigma}_{2} \cdot \mathbf{q}\right)\right. \\
& -i \frac{g_{\Lambda}^{(2)}(-\mathbf{r}, \mathbf{q})}{r} \boldsymbol{\sigma}_{2}\left(\boldsymbol{\sigma}_{1} \cdot \mathbf{q}\right)-i \frac{g_{\Lambda}^{(3)}(\mathbf{r}, \mathbf{q})}{r} \hat{\mathbf{r}}\left(\boldsymbol{\sigma}_{1} \cdot \hat{\mathbf{r}}\right)\left(\boldsymbol{\sigma}_{2} \cdot \mathbf{q}\right)+i \frac{g_{\Lambda}^{(3)}(-\mathbf{r}, \mathbf{q})}{r} \hat{\mathbf{r}}\left(\boldsymbol{\sigma}_{1} \cdot \mathbf{q}\right)\left(\boldsymbol{\sigma}_{2} \cdot \hat{\mathbf{r}}\right) \\
& \left.\left.-g_{\Lambda}^{(4)}(\mathbf{r}, \mathbf{q}) \hat{\mathbf{r}}\left(\boldsymbol{\sigma}_{1} \cdot \mathbf{q}\right)\left(\boldsymbol{\sigma}_{2} \cdot \mathbf{q}\right)-\frac{g_{\Lambda}^{(5)}(\mathbf{r}, \mathbf{q})}{r^{2}} \hat{\mathbf{r}}\left(\boldsymbol{\sigma}_{1} \cdot \hat{\mathbf{r}}\right)\left(\boldsymbol{\sigma}_{2} \cdot \hat{\mathbf{r}}\right)\right]\right\}
\end{aligned}
$$

where $\mathbf{r}=\mathbf{r}_{1}-\mathbf{r}_{2}$ and $\mathbf{R}=\left(\mathbf{r}_{1}+\mathbf{r}_{2}\right) / 2$, and the functions $g_{\Lambda}^{(i)}$ with $i=1, \ldots, 5$ are defined as

$$
\begin{aligned}
& g_{\Lambda}^{(1)}(\mathbf{r}, \mathbf{q})=\int_{-1}^{+1} \mathrm{~d} x \mathrm{e}^{-i x \mathbf{q} \cdot \mathbf{r} / 2}\left(1-r \frac{\mathrm{d}}{\mathrm{d} r}\right) E_{q}(x, r), \\
& g_{\Lambda}^{(2)}(\mathbf{r}, \mathbf{q})=\frac{1}{2} \int_{-1}^{+1} \mathrm{~d} x \mathrm{e}^{-i x \mathbf{q} \cdot \mathbf{r} / 2}(1+x) E_{q}(x, r), \\
& g_{\Lambda}^{(3)}(\mathbf{r}, \mathbf{q})=\frac{1}{2} \int_{-1}^{+1} \mathrm{~d} x \mathrm{e}^{-i x \mathbf{q} \cdot \mathbf{r} / 2}(1+x)\left(1-r \frac{\mathrm{d}}{\mathrm{d} r}\right) E_{q}(x, r), \\
& g_{\Lambda}^{(4)}(\mathbf{r}, \mathbf{q})=\frac{1}{4} \int_{-1}^{+1} \mathrm{~d} x \mathrm{e}^{-i x \mathbf{q} \cdot \mathbf{r} / 2}\left(1-x^{2}\right) E_{q}(x, r), \\
& g_{\Lambda}^{(5)}(\mathbf{r}, \mathbf{q})=\int_{-1}^{+1} \mathrm{~d} x \mathrm{e}^{-i x \mathbf{q} \cdot \mathbf{r} / 2}\left(3-3 r \frac{\mathrm{d}}{\mathrm{d} r}+r^{2} \frac{\mathrm{d}^{2}}{\mathrm{~d} r^{2}}\right) E_{q}(x, r) .
\end{aligned}
$$

The configuration-space representation of the $\mathrm{N}^{2} \mathrm{LO}$ current in Eq. (4.12) reads

$$
\begin{aligned}
\mathbf{j}_{\mathrm{b}-\mathrm{g}}^{\mathrm{N} L O}(\mathbf{q}) & =i \frac{e \mu^{*}}{9 m_{N}} \frac{g_{A} h_{A}}{\Delta F_{\pi}^{2}} \mathrm{e}^{i \mathbf{q} \cdot \mathbf{r}_{1}} \mathbf{q} \times\left[4 \tau_{2, z}\left[h_{S}(r) \boldsymbol{\sigma}_{2}+h_{T}(r) \hat{\mathbf{r}}\left(\boldsymbol{\sigma}_{2} \cdot \hat{\mathbf{r}}\right)\right]\right. \\
& \left.-\left(\boldsymbol{\tau}_{1} \times \boldsymbol{\tau}_{2}\right)_{z}\left[h_{S}(r) \boldsymbol{\sigma}_{1} \times \boldsymbol{\sigma}_{2}+h_{T}(r)\left(\boldsymbol{\sigma}_{1} \times \hat{\mathbf{r}}\right)\left(\boldsymbol{\sigma}_{2} \cdot \hat{\mathbf{r}}\right)\right]\right]+1 \rightleftharpoons 2,
\end{aligned}
$$

where $h_{S}(r)=f_{\Lambda}^{\prime}(r) / r$ and

$$
\begin{aligned}
h_{T}(r)= & f_{\Lambda}^{\prime \prime}(r)-f_{\Lambda}^{\prime}(r) / r=\frac{m_{\pi}^{3}}{8 \pi} \frac{\mathrm{e}^{m_{\pi}^{2} / \Lambda^{2}}}{z_{\pi}^{3}}\left[\phi\left(z_{-}\right) \mathrm{e}^{-z_{\pi}}\left(3+3 z_{\pi}+z_{\pi}^{2}\right)\right. \\
& \left.-\phi\left(z_{+}\right) \mathrm{e}^{z_{\pi}}\left(3-3 z_{\pi}+z_{\pi}^{2}\right)\right]-\frac{\Lambda^{3}}{8 \pi \sqrt{\pi}} \frac{\mathrm{e}^{-z_{\Lambda}^{2} / 4}}{z_{\Lambda}^{2}}\left(6+z_{\Lambda}^{2}\right),
\end{aligned}
$$

and again in the limit $\Lambda \rightarrow \infty$,

$$
h_{S, \infty}(r)=-\frac{m_{\pi}^{3}}{4 \pi} \frac{\mathrm{e}^{-z_{\pi}}}{z_{\pi}^{3}}\left(1+z_{\pi}\right), \quad h_{T, \infty}(r)=\frac{m_{\pi}^{3}}{4 \pi} \frac{\mathrm{e}^{-z_{\pi}}}{z_{\pi}^{3}}\left(3+3 z_{\pi}+z_{\pi}^{2}\right) .
$$




\section{APPENDIX C: ONE-LOOP TWO-BODY CURRENTS WITH $\Delta$ ISOBARS}

We begin by including a single $\Delta$ isobar in the intermediate states. The relevant diagrams are shown in Fig. 9. We find for type a) and b) diagrams:

$$
\begin{aligned}
\text { type a }) & =-e \frac{h_{A}^{2}}{F_{\pi}^{4}}\left(2 \tau_{2, z}-T_{1, z}^{\dagger} \mathbf{T}_{1} \cdot \boldsymbol{\tau}_{2}\right) \int \frac{\left(\mathbf{S}_{1}^{\dagger} \cdot \mathbf{q}_{2}\right) \mathbf{S}_{1}}{\left(\omega_{1}+\Delta\right)\left(\omega_{2}+\Delta\right)\left(\omega_{1}+\omega_{2}\right)}-\text { h.c. }, \\
\text { type b) } & =e \frac{h_{A}^{2}}{F_{\pi}^{4}}\left(2 \tau_{2, z}-T_{1, z}^{\dagger} \mathbf{T}_{1} \cdot \boldsymbol{\tau}_{2}\right) \int\left(\mathbf{q}_{1}-\mathbf{q}_{3}\right)\left(\mathbf{S}_{1}^{\dagger} \cdot \mathbf{q}_{2}\right)\left(\mathbf{S}_{1} \cdot \mathbf{q}_{1}\right) \\
& \times \frac{\omega_{1}+\omega_{2}+\omega_{3}+\Delta}{\left(\omega_{1}+\Delta\right)\left(\omega_{2}+\Delta\right)\left(\omega_{3}+\Delta\right)\left(\omega_{1}+\omega_{2}\right)\left(\omega_{1}+\omega_{3}\right)\left(\omega_{2}+\omega_{3}\right)}-\text { h.c. }
\end{aligned}
$$

where $\mathbf{S}$ and $\mathbf{T}$ are the spin- and isospin-transition operators defined in Eq. (2.10), and $\Delta$ denotes $m_{\Delta}-m_{N}$. The spin-isospin structures can be further simplified and expressed in terms of the Pauli matrices $\boldsymbol{\sigma}$ and $\boldsymbol{\tau}$.

The contributions of type c)-e) diagrams can be written as

$$
\begin{aligned}
& \text { type c) }=\int\left[-\frac{v_{\Delta N}^{\pi \dagger}\left(\mathbf{q}_{2}\right) \mathbf{j}_{\Delta N}^{\pi}\left(\mathbf{q}_{1}\right)}{\Delta}+\mathbf{j}_{\mathrm{c}}^{(-)}\left(\mathbf{q}_{1}, \mathbf{q}_{2}\right)\right]-\text { h.c. } \\
& \text { type d) }=\int\left[-\frac{v_{N \Delta}^{\pi \dagger}\left(\mathbf{q}_{2}\right) \mathbf{j}_{N \Delta}^{\pi}\left(\mathbf{q}_{1}\right)}{\Delta}+\mathbf{j}_{\mathrm{d}}^{(-)}\left(\mathbf{q}_{1}, \mathbf{q}_{2}\right)\right]-\text { h.c. } \\
& \text { type e) }=\int\left[-\frac{v_{\Delta N}^{\pi \dagger}\left(\mathbf{q}_{2}\right) \mathbf{j}_{\Delta N}^{\pi \pi}\left(\mathbf{q}_{1}, \mathbf{q}_{3}\right)}{\Delta}+\mathbf{j}_{\mathrm{e}}^{(-)}\left(\mathbf{q}_{1}, \mathbf{q}_{2}, \mathbf{q}_{3}\right)\right]-\text { h.c. }
\end{aligned}
$$

where we have defined the one-pion-exchange transition potential $N N \rightarrow \Delta N$ as

$$
v_{\Delta N}^{\pi}\left(\mathbf{q}_{2}\right)=-\frac{g_{A} h_{A}}{F_{\pi}^{2}} \frac{\left(\mathbf{S}_{1} \cdot \mathbf{q}_{2}\right)\left(\boldsymbol{\sigma}_{2} \cdot \mathbf{q}_{2}\right)}{q_{2}^{2}+m_{\pi}^{2}} \mathbf{T}_{1} \cdot \boldsymbol{\tau}_{2},
$$

the transition currents $\gamma N N \rightarrow \Delta N$ associated with the seagull $\gamma \pi N \Delta$-coupling and pionin-flight terms respectively as

$$
\begin{aligned}
\mathbf{j}_{\Delta N}^{\pi}\left(\mathbf{q}_{1}\right) & =-i e \frac{g_{A} h_{A}}{F_{\pi}^{2}} \frac{\mathbf{S}_{1}\left(\boldsymbol{\sigma}_{2} \cdot \mathbf{q}_{1}\right)}{q_{1}^{2}+m_{\pi}^{2}}\left(\mathbf{T}_{1} \times \boldsymbol{\tau}_{2}\right)_{z} \\
\mathbf{j}_{\Delta N}^{\pi \pi}\left(\mathbf{q}_{1}, \mathbf{q}_{3}\right) & =i e \frac{g_{A} h_{A}}{F_{\pi}^{2}} \frac{\mathbf{q}_{1}-\mathbf{q}_{3}}{\left(q_{1}^{2}+m_{\pi}^{2}\right)\left(q_{3}^{2}+m_{\pi}^{2}\right)}\left(\mathbf{S}_{1} \cdot \mathbf{q}_{1}\right)\left(\boldsymbol{\sigma}_{2} \cdot \mathbf{q}_{3}\right)\left(\mathbf{T}_{1} \times \boldsymbol{\tau}_{2}\right)_{z}
\end{aligned}
$$

and $v_{N \Delta}^{\pi}$ and $\mathbf{j}_{N \Delta}^{\pi}$ are obtained from $v_{\Delta N}^{\pi}$ and $\mathbf{j}_{\Delta N}^{\pi}$ by the replacements $\mathbf{S} \rightleftharpoons \boldsymbol{\sigma}$ and $\mathbf{T} \rightleftharpoons \boldsymbol{\tau}$. The contributions labeled $\mathbf{j}_{\mathrm{c}, \mathrm{d}, \mathrm{e}}^{(-)}$are given by

$$
\begin{aligned}
\mathbf{j}_{\mathrm{c}}^{(-)}\left(\mathbf{q}_{1}, \mathbf{q}_{2}\right) & =-e \frac{\left(g_{A} h_{A}\right)^{2}}{F_{\pi}^{4}} f_{1}^{\Delta}\left(\omega_{1}, \omega_{2}\right)\left(\mathbf{S}_{1}^{\dagger} \cdot \mathbf{q}_{2}\right) \mathbf{S}_{1}\left[\left(\mathbf{T}_{1}^{\dagger} \times \mathbf{T}_{1}\right)_{z} \boldsymbol{\sigma}_{2} \cdot\left(\mathbf{q}_{1} \times \mathbf{q}_{2}\right)\right. \\
& \left.+\left(2 \tau_{2, z}-T_{1, z}^{\dagger} \mathbf{T}_{1} \cdot \boldsymbol{\tau}_{2}\right) \mathbf{q}_{1} \cdot \mathbf{q}_{2}\right] \\
\mathbf{j}_{\mathrm{d}}^{(-)}\left(\mathbf{q}_{1}, \mathbf{q}_{2}\right) & =i e \frac{\left(g_{A} h_{A}\right)^{2}}{2 F_{\pi}^{4}} f_{1}^{\Delta}\left(\omega_{1}, \omega_{2}\right)\left(\boldsymbol{\sigma}_{1} \cdot \mathbf{q}_{2}\right) \boldsymbol{\sigma}_{1}
\end{aligned}
$$




$$
\begin{aligned}
\times & {\left[\left(\mathbf{S}_{2}^{\dagger} \cdot \mathbf{q}_{1}\right)\left(\mathbf{S}_{2} \cdot \mathbf{q}_{2}\right)\left[\left(\mathbf{T}_{2}^{\dagger} \times \mathbf{T}_{2}\right)_{z}+i\left(2 \tau_{1, z}-\boldsymbol{\tau}_{1} \cdot \mathbf{T}_{2}^{\dagger} T_{2, z}\right)\right]+\text { h.c. }\right],(} \\
\mathbf{j}_{\mathrm{e}}^{(-)}\left(\mathbf{q}_{1}, \mathbf{q}_{2}, \mathbf{q}_{3}\right) & =e \frac{\left(g_{A} h_{A}\right)^{2}}{F_{\pi}^{4}} f_{2}^{\Delta}\left(\omega_{1}, \omega_{2}, \omega_{3}\right)\left(\mathbf{q}_{1}-\mathbf{q}_{3}\right)\left(\mathbf{S}_{1}^{\dagger} \cdot \mathbf{q}_{2}\right)\left(\mathbf{S}_{1} \cdot \mathbf{q}_{1}\right) \\
& \times\left[\left(\mathbf{T}_{1}^{\dagger} \times \mathbf{T}_{1}\right)_{z} \boldsymbol{\sigma}_{2} \cdot\left(\mathbf{q}_{3} \times \mathbf{q}_{2}\right)+\left(2 \tau_{2, z}-T_{1, z}^{\dagger} \mathbf{T}_{1} \cdot \boldsymbol{\tau}_{2}\right) \mathbf{q}_{2} \cdot \mathbf{q}_{3}\right]
\end{aligned}
$$

where the functions $f_{1}^{\Delta}\left(\omega_{1}, \omega_{2}\right)$ and $f_{2}^{\Delta}\left(\omega_{1}, \omega_{2}, \omega_{3}\right)$ denote the following combinations of pion energies and $\Delta N$ mass differences:

$$
\begin{aligned}
& f_{1}^{\Delta}\left(\omega_{1}, \omega_{2}\right)=\frac{\left(\omega_{1}+\omega_{2}+\Delta\right)\left(\omega_{1}+\omega_{2}\right)-\omega_{1} \omega_{2}}{\omega_{1}^{2} \omega_{2}^{2}\left(\omega_{1}+\Delta\right)\left(\omega_{2}+\Delta\right)\left(\omega_{1}+\omega_{2}\right)} \\
& f_{2}^{\Delta}\left(\omega_{1}, \omega_{2}, \omega_{3}\right)=\left[\omega_{1}^{2} \omega_{2}^{2} \omega_{3}^{2}\left(\omega_{1}+\Delta\right)\left(\omega_{2}+\Delta\right)\left(\omega_{3}+\Delta\right)\left(\omega_{1}+\omega_{2}\right)\left(\omega_{1}+\omega_{3}\right)\left(\omega_{2}+\omega_{3}\right)\right]^{-1} \\
& \times\left\{\omega_{1} \omega_{2} \omega_{3}\left(\omega_{1}+\omega_{2}+\omega_{3}\right)^{2}+\Delta^{2}\left(\omega_{1}+\omega_{2}\right)\left(\omega_{1}+\omega_{3}\right)\left(\omega_{2}+\omega_{3}\right)\right. \\
& +\omega_{1}^{2} \omega_{2}^{2}\left(\omega_{1}+\omega_{2}\right)+\omega_{1}^{2} \omega_{3}^{2}\left(\omega_{1}+\omega_{3}\right)+\omega_{2}^{2} \omega_{3}^{2}\left(\omega_{2}+\omega_{3}\right) \\
& +\Delta\left[3 \omega_{1} \omega_{2} \omega_{3}\left(\omega_{1}+\omega_{2}+\omega_{3}\right)+\omega_{1} \omega_{2}\left(\omega_{1}+\omega_{2}\right)^{2}+\omega_{1} \omega_{3}\left(\omega_{1}+\omega_{3}\right)^{2}\right. \\
& \left.\left.+\omega_{2} \omega_{3}\left(\omega_{2}+\omega_{3}\right)^{2}\right]\right\} \text {. }
\end{aligned}
$$
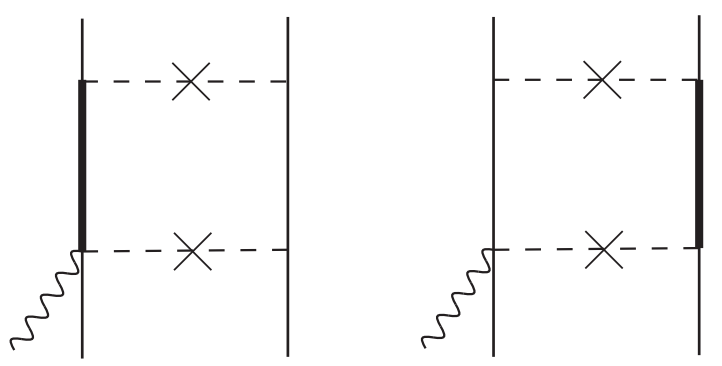

FIG. 13: Direct box diagrams with a single $\Delta$ isobar in the intermediate states. See text for discussion. Dashed lines with a cross represent $v_{\Delta N}$ transition potentials, or $\mathbf{j}_{\Delta N}^{\pi}$ and $\mathbf{j}_{\Delta N}^{\pi \pi}$ transition currents.

At this stage, it is useful to comment on the structure of the contributions in Eqs. (C3)(C5). The first terms on the r.h.s. of each of these equations are represented by the diagrams in Fig. 13, i.e. (from top to bottom) an instantaneous interaction mediated by a transition potential, a $\Delta$-nucleon energy denominator taken in the static limit, and a two-body current inducing excitation of a single $\Delta$. They have a simple interpretation [15]: for example, the type c) in Fig. 9 is the matrix element $\left\langle\Delta N\left|\mathbf{j}_{\pi \Delta N}\right| N N\right\rangle$ evaluated by treating the final $|\Delta N\rangle$ state in first order perturbation theory,

$$
|\Delta N\rangle=|N N\rangle+\sum_{\Delta^{\prime} N^{\prime}}\left|\Delta^{\prime} N^{\prime}\right\rangle \frac{\left\langle\Delta^{\prime} N^{\prime}\left|v^{\Delta N}\right| N N\right\rangle}{E_{N N}-E_{\Delta^{\prime} N^{\prime}}} \simeq\left(1-\frac{v_{\Delta N}^{\pi}}{\Delta}\right)|N N\rangle .
$$

One additional feature of these terms is that their configuration-space representations are particularly simple, since they are given, for example for type c), by the product 
$-v_{\Delta N}^{\pi}(\mathbf{r}) \mathbf{j}_{\Delta N}^{\pi}(\mathbf{q}) / \Delta$, and similarly for type d) and e). Here $v_{\Delta N}^{\pi}(\mathbf{r})$ and $\mathbf{j}_{\Delta N}^{\pi}(\mathbf{q})$ are the configuration-space representations of, respectively, the transition potential and the current in Eq. (C7).

The remaining terms $\mathbf{j}_{\mathrm{c}, \mathrm{d}, \mathrm{e}}^{(-)}$represent corrections to this picture, arising from crossed-box diagrams. In particular, the functions $f_{1}^{\Delta}\left(\omega_{1}, \omega_{2}\right)$ and $f_{2}^{\Delta}\left(\omega_{1}, \omega_{2}, \omega_{3}\right)$ give, up to pion-energy factors from field normalizations, the sum of the energy denominators for the six and thirty crossed-box diagrams from, respectively, type c)-d) and e) contributions,

$$
\begin{aligned}
f_{1}^{\Delta}\left(\omega_{1}, \omega_{2}\right) & =-\frac{1}{2 \omega_{1} \omega_{2}}[\text { sum of } 6 \text { type c) or d) crossed-box diagrams }], \\
f_{2}^{\Delta}\left(\omega_{1}, \omega_{2}, \omega_{3}\right) & =\frac{1}{4 \omega_{1} \omega_{2} \omega_{3}}[\text { sum of } 30 \text { type e) crossed-box diagrams }] .
\end{aligned}
$$

Lastly, the contributions of type f)-i) diagrams vanish, while those of type j)-k) are written as

$$
\begin{gathered}
\text { type j) }=\int\left[-\frac{v_{\Delta N}^{\mathrm{c} \dagger} \mathbf{j}_{\Delta N}^{\pi \pi}\left(\mathbf{q}_{1}, \mathbf{q}_{2}\right)}{\Delta}+\mathbf{j}_{\mathrm{j}}^{(-)}\left(\mathbf{q}_{1}, \mathbf{q}_{2}\right)\right]-\text { h.c. }, \\
\text { type k) }=-i e \frac{g_{A} h_{A} D_{T}}{2 F_{\pi}^{2}} \int\left(\mathbf{q}_{1}-\mathbf{q}_{2}\right)\left[f_{1}^{\Delta}\left(\omega_{1}, \omega_{2}\right)\left(\boldsymbol{\sigma}_{1} \cdot \mathbf{q}_{2}\right)\left(\mathbf{S}_{1}^{\dagger} \cdot \boldsymbol{\sigma}_{2}\right)\left(\mathbf{S}_{1} \cdot \mathbf{q}_{1}\right)\right. \\
\times\left[\mathbf{T}_{1}^{\dagger} \cdot \boldsymbol{\tau}_{2}\left(\mathbf{T}_{1} \times \boldsymbol{\tau}_{1}\right)_{z}-i\left[\left(\mathbf{T}_{1}^{\dagger} \times \mathbf{T}_{1}\right) \times \boldsymbol{\tau}_{2}\right]_{z}\right]+\frac{1}{\Delta \omega_{1}^{2} \omega_{2}^{2}} \mathbf{S}_{1}^{\dagger} \cdot \boldsymbol{\sigma}_{2}\left(\mathbf{S}_{1} \cdot \mathbf{q}_{1}\right)\left(\boldsymbol{\sigma}_{1} \cdot \mathbf{q}_{2}\right) \\
\left.\times \mathbf{T}_{1}^{\dagger} \cdot \boldsymbol{\tau}_{2}\left(\mathbf{T}_{1} \times \boldsymbol{\tau}_{1}\right)_{z}\right] \text {-h.c. }
\end{gathered}
$$

where the contact (momentum-independent) transition potential $v_{\Delta N}^{\mathrm{c}}$ has been defined as

$$
v_{\Delta N}^{\mathrm{c}}=D_{T} \mathbf{T}_{1} \cdot \boldsymbol{\tau}_{2} \mathbf{S}_{1} \cdot \boldsymbol{\sigma}_{2},
$$

and the current $\mathbf{j}_{\mathrm{j}}^{(-)}$is given by

$$
\begin{aligned}
\mathbf{j}_{j}^{(-)}\left(\mathbf{q}_{1}, \mathbf{q}_{2}\right) & =i e \frac{g_{A} h_{A}}{2 F_{\pi}^{2}}\left(\mathbf{q}_{1}-\mathbf{q}_{2}\right) f_{1}^{\Delta}\left(\omega_{1}, \omega_{2}\right)\left[v_{\Delta N}^{\mathrm{c} \dagger}\left(\mathbf{T}_{1} \times \boldsymbol{\tau}_{2}\right)_{z}\left(\boldsymbol{\sigma}_{2} \cdot \mathbf{q}_{2}\right)-D_{T}\left[\mathbf{T}_{1}^{\dagger} \cdot \boldsymbol{\tau}_{2}\left(\mathbf{T}_{1} \times \boldsymbol{\tau}_{2}\right)_{z}\right.\right. \\
& \left.\left.-2 i\left(2 \tau_{2, z}-T_{1, z}^{\dagger} \mathbf{T}_{1} \cdot \boldsymbol{\tau}_{2}\right)\right]\left(\boldsymbol{\sigma}_{2} \cdot \mathbf{q}_{2}\right)\left(\mathbf{S}_{1}^{\dagger} \cdot \boldsymbol{\sigma}_{2}\right)\right]\left(\mathbf{S}_{1} \cdot \mathbf{q}_{1}\right)-\text { h.c. }
\end{aligned}
$$

The next set of contributions we consider includes two $\Delta$ isobars in the intermediate states, the relevant diagrams are displayed in Fig. 10, In analogy to Eqs. (C3) $-($ C5) $)$, we write:

$$
\begin{aligned}
\text { type l) } & =\int\left[-\frac{v_{\Delta \Delta}^{\pi \dagger}\left(\mathbf{q}_{2}\right) \mathbf{j}_{\Delta \Delta}^{\pi}\left(\mathbf{q}_{1}\right)}{2 \Delta}+\mathbf{j}_{1}^{(-)}\left(\mathbf{q}_{1}, \mathbf{q}_{2}\right)\right]-\text { h.c. } \\
\text { type } \mathrm{m}) & =\int\left[-\frac{v_{\Delta \Delta}^{\pi \dagger}\left(\mathbf{q}_{2}\right) \mathbf{j}_{\Delta \Delta}^{\pi \pi}\left(\mathbf{q}_{1}, \mathbf{q}_{3}\right)}{2 \Delta}+\mathbf{j}_{\mathrm{m}}^{(-)}\left(\mathbf{q}_{1}, \mathbf{q}_{2}, \mathbf{q}_{3}\right)\right]-\text { h.c. },
\end{aligned}
$$


where the transition potential $v_{\Delta \Delta}^{\pi}$ and currents $\mathbf{j}_{\Delta \Delta}^{\pi}$ and $\mathbf{j}_{\Delta \Delta}^{\pi \pi}$ are obtained from Eqs. (C66)(C8) by the replacements $g_{A} \rightarrow h_{A}$ and $\boldsymbol{\sigma}_{2}\left(\boldsymbol{\tau}_{2}\right) \rightarrow \mathbf{S}_{2}\left(\mathbf{T}_{2}\right)$, and

$$
\begin{aligned}
\mathbf{j}_{1}^{(-)}\left(\mathbf{q}_{1}, \mathbf{q}_{2}\right) & =-v_{\Delta \Delta}^{\pi \dagger}\left(\mathbf{q}_{2}\right) \mathbf{j}_{\Delta \Delta}^{\pi}\left(\mathbf{q}_{1}\right)\left[f_{3}^{\Delta}\left(\omega_{1}, \omega_{2}\right)-\frac{1}{2 \Delta}\right]-i e \frac{h_{A}^{4}}{2 F_{\pi}^{4}} f_{4}^{\Delta}\left(\omega_{1}, \omega_{2}\right)\left(\mathbf{S}_{1}^{\dagger} \cdot \mathbf{q}_{2}\right) \mathbf{S}_{1} \\
& \times\left[\left(\mathbf{S}_{2}^{\dagger} \cdot \mathbf{q}_{1}\right)\left(\mathbf{S}_{2} \cdot \mathbf{q}_{2}\right)\left[\left(\mathbf{T}_{1}^{\dagger} \times \mathbf{T}_{2}^{\dagger}\right)_{z} \mathbf{T}_{1} \cdot \mathbf{T}_{2}-2\left(\mathbf{T}_{1}^{\dagger} \times \mathbf{T}_{1}\right)_{z}+\mathbf{T}_{2}^{\dagger} \cdot\left(\mathbf{T}_{1}^{\dagger} \times \mathbf{T}_{1}\right) T_{2, z}\right]\right. \\
& \left.-\left(\mathbf{S}_{2}^{\dagger} \cdot \mathbf{q}_{2}\right)\left(\mathbf{S}_{2} \cdot \mathbf{q}_{1}\right) \mathbf{T}_{1}^{\dagger} \cdot \mathbf{T}_{2}^{\dagger}\left(\mathbf{T}_{1} \times \mathbf{T}_{2}\right)_{z}\right] \\
\mathbf{j}_{\mathrm{m}}^{(-)}\left(\mathbf{q}_{1}, \mathbf{q}_{2}, \mathbf{q}_{3}\right) & =-v_{\Delta \Delta}^{\pi \dagger}\left(\mathbf{q}_{2}\right) \mathbf{j}_{\Delta \Delta}^{\pi \pi}\left(\mathbf{q}_{1}, \mathbf{q}_{3}\right)\left[f_{5}^{\Delta}\left(\omega_{1}, \omega_{2}, \omega_{3}\right)-\frac{1}{2 \Delta}\right] \\
& +i e \frac{h_{A}^{4}}{2 F_{\pi}^{4}}\left(\mathbf{q}_{1}-\mathbf{q}_{3}\right) f_{6}^{\Delta}\left(\omega_{1}, \omega_{2}, \omega_{3}\right)\left(\mathbf{S}_{1}^{\dagger} \cdot \mathbf{q}_{2}\right)\left(\mathbf{S}_{1} \cdot \mathbf{q}_{1}\right) \\
& \times\left[\left(\mathbf{S}_{2}^{\dagger} \cdot \mathbf{q}_{3}\right)\left(\mathbf{S}_{2} \cdot \mathbf{q}_{2}\right)\left[\left(\mathbf{T}_{1}^{\dagger} \times \mathbf{T}_{2}^{\dagger}\right)_{z} \mathbf{T}_{1} \cdot \mathbf{T}_{2}-2\left(\mathbf{T}_{1}^{\dagger} \times \mathbf{T}_{1}\right)_{z}+\mathbf{T}_{2}^{\dagger} \cdot\left(\mathbf{T}_{1}^{\dagger} \times \mathbf{T}_{1}\right) T_{2, z}\right]\right. \\
& \left.-\left(\mathbf{S}_{2}^{\dagger} \cdot \mathbf{q}_{2}\right)\left(\mathbf{S}_{2} \cdot \mathbf{q}_{3}\right) \mathbf{T}_{1}^{\dagger} \cdot \mathbf{T}_{2}^{\dagger}\left(\mathbf{T}_{1} \times \mathbf{T}_{2}\right)_{z}\right] .
\end{aligned}
$$

The functions $f_{3}^{\Delta}\left(\omega_{1}, \omega_{2}\right)$ and $f_{5}^{\Delta}\left(\omega_{1}, \omega_{2}, \omega_{3}\right)$ are defined as

$$
\begin{aligned}
f_{3}^{\Delta}\left(\omega_{1}, \omega_{2}\right) & =-\frac{\omega_{1} \omega_{2}}{4} \text { [sum of } 12 \text { type } \mathrm{m} \text { ) direct and crossed-box diagrams ], } \\
f_{5}^{\Delta}\left(\omega_{1}, \omega_{2}, \omega_{3}\right) & \left.=\frac{\omega_{1} \omega_{2} \omega_{3}}{8} \text { [sum of } 60 \text { type } n\right) \text { direct and crossed-box diagrams ], }
\end{aligned}
$$

while $f_{4}^{\Delta}\left(\omega_{1}, \omega_{2}\right)$ and $f_{6}^{\Delta}\left(\omega_{1}, \omega_{2}, \omega_{3}\right)$ as in Eqs. (C15) and (C16), but for diagrams of type 1) and $\mathrm{m})$, respectively. They are explicitly given by

$$
\begin{aligned}
& f_{3}^{\Delta}\left(\omega_{1}, \omega_{2}\right)=\frac{\omega_{1} \omega_{2}\left[2 \Delta^{3}+\left(4 \Delta^{2}+\omega_{1} \omega_{2}\right)\left(\omega_{1}+\omega_{2}\right)+2 \Delta\left(\omega_{1}+\omega_{2}\right)^{2}\right]}{2 \Delta\left(\omega_{1}+\omega_{2}\right)\left(\omega_{1}+\Delta\right)^{2}\left(\omega_{2}+\Delta\right)^{2}} \\
& f_{4}^{\Delta}\left(\omega_{1}, \omega_{2}\right)=\frac{\Delta^{2}+\omega_{1}^{2}+\omega_{1} \omega_{2}+\omega_{2}^{2}+2 \Delta\left(\omega_{1}+\omega_{2}\right)}{\omega_{1} \omega_{2}\left(\omega_{1}+\omega_{2}\right)\left(\omega_{1}+\Delta\right)^{2}\left(\omega_{2}+\Delta\right)^{2}}
\end{aligned}
$$

and

$$
\begin{aligned}
f_{5}^{\Delta}\left(\omega_{1}, \omega_{2}, \omega_{3}\right) & =\frac{\omega_{1} \omega_{2} \omega_{3}}{2}\left[\frac{\left(\omega_{1}+\omega_{2}+\omega_{3}\right)\left(\omega_{1}+\omega_{2}+\omega_{3}+\Delta\right)}{\left(\omega_{1}+\Delta\right)\left(\omega_{2}+\Delta\right)\left(\omega_{3}+\Delta\right)\left(\omega_{1}+\omega_{2}\right)\left(\omega_{1}+\omega_{3}\right)\left(\omega_{2}+\omega_{3}\right)}\right. \\
& \left.+\frac{1}{\Delta\left(\omega_{1}+\Delta\right)\left(\omega_{2}+\Delta\right)\left(\omega_{3}+\Delta\right)}+\omega_{1} \omega_{2} \omega_{3} f_{6}^{\Delta}\left(\omega_{1}, \omega_{2}, \omega_{3}\right)\right] \\
f_{6}^{\Delta}\left(\omega_{1}, \omega_{2}, \omega_{3}\right) & =\frac{1}{\omega_{1} \omega_{2} \omega_{3}\left(\omega_{1}+\omega_{2}\right)\left(\omega_{1}+\omega_{3}\right)\left(\omega_{2}+\omega_{3}\right)}\left[\frac{1}{\left(\omega_{1}+\Delta\right)^{2}}+\frac{1}{\left(\omega_{2}+\Delta\right)^{2}}\right. \\
& \left.+\frac{1}{\left(\omega_{3}+\Delta\right)^{2}}\right]+\frac{\omega_{1} \omega_{2} \omega_{3}-\Delta^{2}\left(\omega_{1}+\omega_{2}+\omega_{3}\right)}{\omega_{1} \omega_{2} \omega_{3}\left(\omega_{1}+\omega_{2}\right)\left(\omega_{1}+\omega_{3}\right)\left(\omega_{2}+\omega_{3}\right)} \\
& \times\left[\frac{1}{\left(\omega_{1}+\Delta\right)^{2}\left(\omega_{2}+\Delta\right)^{2}}+\frac{1}{\left(\omega_{1}+\Delta\right)^{2}\left(\omega_{3}+\Delta\right)^{2}}+\frac{1}{\left(\omega_{2}+\Delta\right)^{2}\left(\omega_{3}+\Delta\right)^{2}}\right] \\
& +\frac{\Delta^{4}\left(\omega_{1}+\omega_{2}+\omega_{3}\right)+\omega_{1} \omega_{2} \omega_{3}\left(\omega_{1} \omega_{2}+\omega_{1} \omega_{3}+\omega_{2} \omega_{3}-6 \Delta^{2}\right)}{\omega_{1} \omega_{2} \omega_{3}\left(\omega_{1}+\Delta\right)^{2}\left(\omega_{2}+\Delta\right)^{2}\left(\omega_{3}+\Delta\right)^{2}\left(\omega_{1}+\omega_{2}\right)\left(\omega_{1}+\omega_{3}\right)\left(\omega_{2}+\omega_{3}\right)} \cdot(\mathrm{C} 3 \mathrm{C}
\end{aligned}
$$


We observe that, in contrast to the case of a single $\Delta$, the energy denominators for the direct and crossed box diagrams do not add up to $1 /(2 \Delta)$ - as one would have naively expectedindeed, that is the reason for including this term within the square brackets of Eqs. (C23) and ( $\mathrm{C} 24)$.

Next, we consider diagrams $\mathrm{n}$ )-s) in Fig. 10, which involve contact terms with two $\Delta$ 's. The contributions of type $n$ )-p) vanish, while those of type q)-s) read:

$$
\begin{aligned}
\text { type q })= & i e \frac{h_{A}^{2}}{2 F_{\pi}^{2}}\left(\mathbf{T}_{1}^{\dagger} \times \mathbf{T}_{1}\right)_{z} \int\left(\mathbf{q}_{1}-\mathbf{q}_{2}\right) f_{4}^{\Delta}\left(\omega_{1}, \omega_{2}\right)\left[C_{S}^{\prime}\left(\mathbf{S}_{1}^{\dagger} \cdot \mathbf{q}_{2}\right)\left(\mathbf{S}_{1} \cdot \mathbf{q}_{1}\right)\right. \\
& \left.+C_{T}^{\prime}\left(\mathbf{S}_{1}^{\dagger} \cdot \mathbf{q}_{2}\right)\left(\mathbf{\Sigma}_{1} \cdot \boldsymbol{\sigma}_{2}\right)\left(\mathbf{S}_{1} \cdot \mathbf{q}_{1}\right)\right] \\
\text { type } \mathrm{r})= & -i e \frac{h_{A}^{2}}{2 F_{\pi}^{2}} \frac{D_{T}^{\prime}}{\Delta}\left(\mathbf{T}_{1}^{\dagger} \cdot \mathbf{T}_{2}^{\dagger}\right)\left(\mathbf{T}_{1} \times \mathbf{T}_{2}\right)_{z}\left(\mathbf{S}_{1}^{\dagger} \cdot \mathbf{S}_{2}^{\dagger}\right) \int\left(\mathbf{q}_{1}-\mathbf{q}_{2}\right)\left(\mathbf{S}_{1} \cdot \mathbf{q}_{1}\right)\left(\mathbf{S}_{2} \cdot \mathbf{q}_{2}\right) \\
\times & \frac{\omega_{1}+\omega_{2}+\Delta}{\omega_{1} \omega_{2}\left(\omega_{1}+\omega_{2}\right)\left(\omega_{1}+\Delta\right)\left(\omega_{2}+\Delta\right)}-\text { h.c. }, \\
\text { type s)=} & -i e \frac{h_{A}^{2} D_{T}^{\prime \prime}}{2 F_{\pi}^{2}}\left[\mathbf{T}_{1}^{\dagger} \cdot \mathbf{T}_{2}^{\dagger}\left(\mathbf{T}_{1} \times \mathbf{T}_{2}\right)_{z}+2\left(\mathbf{T}_{1}^{\dagger} \times \mathbf{T}_{1}\right)_{z}-T_{2, z}^{\dagger} \mathbf{T}_{2} \cdot\left(\mathbf{T}_{1}^{\dagger} \times \mathbf{T}_{1}\right)\right] \\
& \int\left(\mathbf{q}_{1}-\mathbf{q}_{2}\right) f_{4}^{\Delta}\left(\omega_{1}, \omega_{2}\right)\left(\mathbf{S}_{1}^{\dagger} \cdot \mathbf{q}_{1}\right)\left(\mathbf{S}_{1} \cdot \mathbf{S}_{2}^{\dagger}\right)\left(\mathbf{S}_{2} \cdot \mathbf{q}_{2}\right)-\text { h.c. } .
\end{aligned}
$$

\section{APPENDIX D: CURRENTS FROM CONTACT INTERACTIONS}

In this Appendix we list the four-nucleon contact interaction Hamiltonians involving two gradients of the nucleon fields. Minimal substitution,

$$
\nabla N(\mathbf{x}) \rightarrow\left[\nabla-i e e_{N} \mathbf{A}(\mathbf{x})\right] N(\mathbf{x})
$$

in the nucleon-derivative couplings then leads to the corresponding electromagneticinteraction Hamiltonians, which are listed as well. In the last section of this Appendix we report the expressions for the vertices induced by these Hamiltonians.

\section{Four-nucleon contact interaction Hamiltonians}

The four-nucleon contact interaction Hamiltonians with two gradients acting on the nucleon fields have the following expressions [2, 3] :

$$
\begin{aligned}
& H_{\mathrm{CT} 2 \mathrm{D}, 1}=C_{1}^{\prime} \int \mathrm{d} \mathbf{x}\left[\left[N^{\dagger}(\mathbf{x}) \nabla N(\mathbf{x})\right]^{2}+\left[[\nabla N(\mathbf{x})]^{\dagger} N(\mathbf{x})\right]^{2}\right] \\
& H_{\mathrm{CT} 2 \mathrm{D}, 2}=C_{2}^{\prime} \int \mathrm{d} \mathbf{x}\left[N^{\dagger}(\mathbf{x}) \nabla N(\mathbf{x})\right] \cdot\left[[\nabla N(\mathbf{x})]^{\dagger} N(\mathbf{x})\right] \\
& H_{\mathrm{CT} 2 \mathrm{D}, 3}=C_{3}^{\prime} \int \mathrm{d} \mathbf{x}\left[N^{\dagger}(\mathbf{x}) N(\mathbf{x})\right]\left[N^{\dagger}(\mathbf{x}) \nabla^{2} N(\mathbf{x})+\left[\nabla^{2} N(\mathbf{x})\right]^{\dagger} N(\mathbf{x})\right] \\
& H_{\mathrm{CT} 2 \mathrm{D}, 4}=i C_{4}^{\prime} \int \mathrm{d} \mathbf{x}\left[\left[N^{\dagger}(\mathbf{x}) \nabla N(\mathbf{x})\right] \cdot\left[[\nabla N(\mathbf{x})]^{\dagger} \times \boldsymbol{\sigma} N(\mathbf{x})\right]\right.
\end{aligned}
$$




$$
\begin{aligned}
& \left.+\left[[\nabla N(\mathbf{x})]^{\dagger} N(\mathbf{x})\right] \cdot\left[N^{\dagger}(\mathbf{x}) \boldsymbol{\sigma} \times \nabla N(\mathbf{x})\right]\right], \\
& H_{\mathrm{CT} 2 \mathrm{D}, 5}=i C_{5}^{\prime} \int \mathrm{d} \mathbf{x}\left[N^{\dagger}(\mathbf{x}) N(\mathbf{x})\right]\left[[\nabla N(\mathbf{x})]^{\dagger} \cdot \boldsymbol{\sigma} \times \nabla N(\mathbf{x})\right], \\
& H_{\mathrm{CT} 2 \mathrm{D}, 6}=i C_{6}^{\prime} \int \mathrm{d} \mathbf{x}\left[N^{\dagger}(\mathbf{x}) \boldsymbol{\sigma} N(\mathbf{x})\right] \cdot\left[[\nabla N(\mathbf{x})]^{\dagger} \times \nabla N(\mathbf{x})\right], \\
& H_{\mathrm{CT} 2 \mathrm{D}, 7}=\left(C_{7}^{\prime} \delta_{i k} \delta_{j l}+C_{8}^{\prime} \delta_{i l} \delta_{k j}+C_{9}^{\prime} \delta_{i j} \delta_{k l}\right) \\
& \int \mathrm{d} \mathbf{x}\left[\left[N^{\dagger}(\mathbf{x}) \sigma_{k} \partial_{i} N(\mathbf{x})\right]\left[N^{\dagger}(\mathbf{x}) \sigma_{l} \partial_{j} N(\mathbf{x})\right]\right. \\
& \left.+\left[\left[\partial_{i} N(\mathbf{x})\right]^{\dagger} \sigma_{k} N(\mathbf{x})\right]\left[\left[\partial_{j} N(\mathbf{x})\right]^{\dagger} \sigma_{l} N(\mathbf{x})\right]\right], \\
& H_{\mathrm{CT} 2 \mathrm{D}, 8}=\left(C_{10}^{\prime} \delta_{i k} \delta_{j l}+C_{11}^{\prime} \delta_{i l} \delta_{k j}+C_{12}^{\prime} \delta_{i j} \delta_{k l}\right) \\
& \int \mathrm{d} \mathbf{x}\left[N^{\dagger}(\mathbf{x}) \sigma_{k} \partial_{i} N(\mathbf{x})\right]\left[\left[\partial_{j} N(\mathbf{x})\right]^{\dagger} \sigma_{l} N(\mathbf{x})\right], \\
& H_{\mathrm{CT} 2 \mathrm{D}, 9}=\left(\frac{1}{2} C_{13}^{\prime}\left(\delta_{i k} \delta_{j l}+\delta_{i l} \delta_{k j}\right)+C_{14}^{\prime} \delta_{i j} \delta_{k l}\right) \\
& \int \mathrm{d} \mathbf{x}\left[\left[\partial_{i} N(\mathbf{x})\right]^{\dagger} \sigma_{k} \partial_{j} N(\mathbf{x})+\left[\partial_{j} N(\mathbf{x})\right]^{\dagger} \sigma_{k} \partial_{i} N(\mathbf{x})\right]\left[N^{\dagger}(\mathbf{x}) \sigma_{l} N(\mathbf{x})\right]
\end{aligned}
$$

\section{Contact electromagnetic-interaction Hamiltonians}

Minimal substitution leads to the following contact electromagnetic-interaction Hamiltonians:

$$
\begin{aligned}
& H_{\mathrm{CT} \gamma, 1}=-i e C_{1}^{\prime} \int \mathrm{d} \mathbf{x} \mathbf{A}(\mathbf{x}) \cdot\left[\left[N^{\dagger}(\mathbf{x})(\vec{\nabla}-\overleftarrow{\nabla}) N(\mathbf{x})\right]\left[N^{\dagger}(\mathbf{x}) e_{N} N(\mathbf{x})\right]\right. \\
& \left.+\left[N^{\dagger}(\mathbf{x}) e_{N} N(\mathbf{x})\right]\left[N^{\dagger}(\mathbf{x})(\vec{\nabla}-\overleftarrow{\nabla}) N(\mathbf{x})\right]\right], \\
& H_{\mathrm{CT} \gamma, 2}=-i e C_{2}^{\prime} \int \mathrm{d} \mathbf{x} \mathbf{A}(\mathbf{x}) \cdot\left[\left[N^{\dagger}(\mathbf{x}) e_{N} N(\mathbf{x})\right]\left[[\nabla N(\mathbf{x})]^{\dagger} N(\mathbf{x})\right]\right. \\
& \left.-\left[N^{\dagger}(\mathbf{x}) \nabla N(\mathbf{x})\right]\left[N^{\dagger}(\mathbf{x}) e_{N} N(\mathbf{x})\right]\right], \\
& H_{\mathrm{CT} \gamma, 3}=-i e C_{3}^{\prime} \int \mathrm{d} \mathbf{x} \mathbf{A}(\mathbf{x}) \cdot\left[2 N^{\dagger}(\mathbf{x}) N(\mathbf{x})\right]\left[N^{\dagger}(\mathbf{x})(\vec{\nabla}-\overleftarrow{\nabla}) e_{N} N(\mathbf{x})\right], \\
& H_{\mathrm{CT} \gamma, 4}=e C_{4}^{\prime} \int \mathrm{d} \mathbf{x} \mathbf{A}(\mathbf{x}) \cdot\left[\left[N^{\dagger}(\mathbf{x})(\vec{\nabla}+\overleftarrow{\nabla}) N(\mathbf{x})\right] \times\left[N^{\dagger}(\mathbf{x}) \boldsymbol{\sigma} e_{N} N(\mathbf{x})\right]\right. \\
& \left.+\left[N^{\dagger}(\mathbf{x}) e_{N} N(\mathbf{x})\right]\left[N^{\dagger}(\mathbf{x})(\vec{\nabla}+\overleftarrow{\nabla}) \times \boldsymbol{\sigma} N(\mathbf{x})\right]\right], \\
& H_{\mathrm{CT} \gamma, 5}=e C_{5}^{\prime} \int \mathrm{d} \mathbf{x} \mathbf{A}(\mathbf{x}) \cdot\left[N^{\dagger}(\mathbf{x}) N(\mathbf{x})\right]\left[N^{\dagger}(\mathbf{x})(\vec{\nabla}+\overleftarrow{\nabla}) \times \boldsymbol{\sigma} e_{N} N(\mathbf{x})\right], \\
& H_{\mathrm{CT} \gamma, 6}=e C_{6}^{\prime} \int \mathrm{d} \mathbf{x} \mathbf{A}(\mathbf{x}) \cdot\left[N^{\dagger}(\mathbf{x}) \boldsymbol{\sigma} N(\mathbf{x})\right] \times\left[N^{\dagger}(\mathbf{x})(\vec{\nabla}+\overleftarrow{\nabla}) e_{N} N(\mathbf{x})\right], \\
& H_{\mathrm{CT} \gamma, 7}=-i e\left(C_{7}^{\prime} \delta_{i k} \delta_{j l}+C_{8}^{\prime} \delta_{i l} \delta_{k j}+C_{9}^{\prime} \delta_{i j} \delta_{k l}\right) \\
& \int \mathrm{d} \mathbf{x}\left[A_{j}(\mathbf{x})\left[N^{\dagger}(\mathbf{x})\left(\overrightarrow{\partial_{i}}-\overleftarrow{\partial_{i}}\right) \sigma_{k} N(\mathbf{x})\right]\left[N^{\dagger}(\mathbf{x}) \sigma_{l} e_{N} N(\mathbf{x})\right]\right. \\
& \left.+A_{i}(\mathbf{x})\left[N^{\dagger}(\mathbf{x}) \sigma_{k} e_{N} N(\mathbf{x})\right]\left[N^{\dagger}(\mathbf{x})\left(\overrightarrow{\partial_{j}}-\overleftarrow{\delta_{j}}\right) \sigma_{l} N(\mathbf{x})\right]\right], \\
& H_{\mathrm{CT} \gamma, 8}=\quad \text { ie }\left(C_{10}^{\prime} \delta_{i k} \delta_{j l}+C_{11}^{\prime} \delta_{i l} \delta_{k j}+C_{12}^{\prime} \delta_{i j} \delta_{k l}\right)
\end{aligned}
$$




$$
\begin{array}{r}
\int \mathrm{d} \mathbf{x}\left[A_{j}(\mathbf{x})\left[N^{\dagger}(\mathbf{x}) \sigma_{k} \partial_{i} N(\mathbf{x})\right]\left[N^{\dagger}(\mathbf{x}) \sigma_{l} e_{N} N(\mathbf{x})\right]\right. \\
\left.-A_{i}(\mathbf{x})\left[N^{\dagger}(\mathbf{x}) \sigma_{k} e_{N} N(\mathbf{x})\right]\left[\left[\partial_{j} N(\mathbf{x})\right]^{\dagger} \sigma_{l} N(\mathbf{x})\right]\right] \\
H_{\mathrm{CT} \gamma, 9}=i e\left(\frac{1}{2} C_{13}^{\prime}\left(\delta_{i k} \delta_{j l}+\delta_{i l} \delta_{k j}\right)+C_{14}^{\prime} \delta_{i j} \delta_{k l}\right) \\
\int \mathrm{d} \mathbf{x}\left[A_{j}(\mathbf{x})\left[N^{\dagger}(\mathbf{x})\left(\overrightarrow{\partial_{i}}-\overleftarrow{\partial_{i}}\right) \sigma_{k} e_{N} N(\mathbf{x})\right]\left[N^{\dagger}(\mathbf{x}) \sigma_{l} N(\mathbf{x})\right]\right. \\
\left.+A_{i}(\mathbf{x})\left[N^{\dagger}(\mathbf{x})\left(\overrightarrow{\partial_{j}}-\overleftarrow{\partial_{j}}\right) \sigma_{k} e_{N} N(\mathbf{x})\right]\left[N^{\dagger}(\mathbf{x}) \sigma_{l} N(\mathbf{x})\right]\right] .
\end{array}
$$

\section{Contact interaction vertices}

The vertices induced by the contact electromagnetic-interaction Hamiltonians are listed below. The notation is the same as in Appendix A, but for

$$
\left\langle H_{\mathrm{CT} \gamma, i}\right\rangle \equiv\left\langle\mathbf{p}_{1}^{\prime}, \chi_{1}^{\prime} ; \mathbf{p}_{2}^{\prime}, \chi_{2}^{\prime}\left|H_{\mathrm{CT} \gamma, i}\right| \mathbf{p}_{1}, \chi_{1} ; \mathbf{p}_{2}, \chi_{2} ; \mathbf{q}, \lambda\right\rangle, \quad i=1, \ldots, 9
$$

and

$$
\begin{aligned}
& \left\langle H_{\mathrm{CT} \gamma, 1}\right\rangle=2 e C_{1}^{\prime}\left[e_{1}\left(\mathbf{p}_{2}+\mathbf{p}_{2}^{\prime}\right)+e_{2}\left(\mathbf{p}_{1}+\mathbf{p}_{1}^{\prime}\right)\right] \cdot \frac{\hat{\mathbf{e}}_{\mathbf{q} \lambda}}{\sqrt{2 \omega_{q}}} \\
& \left\langle H_{\mathrm{CT} \gamma, 2}\right\rangle=-e C_{2}^{\prime}\left[e_{1}\left(\mathbf{p}_{2}+\mathbf{p}_{2}^{\prime}\right)+e_{2}\left(\mathbf{p}_{1}+\mathbf{p}_{1}^{\prime}\right)\right] \cdot \frac{\hat{\mathbf{e}}_{\mathbf{q} \lambda}}{\sqrt{2 \omega_{q}}} \\
& \left\langle H_{\mathrm{CT} \gamma, 3}\right\rangle=2 e C_{3}^{\prime}\left[e_{1}\left(\mathbf{p}_{1}+\mathbf{p}_{1}^{\prime}\right)+e_{2}\left(\mathbf{p}_{2}+\mathbf{p}_{2}^{\prime}\right)\right] \cdot \frac{\hat{\mathbf{e}}_{\mathbf{q} \lambda}}{\sqrt{2 \omega_{q}}}, \\
& \left\langle H_{\mathrm{CT} \gamma, 4}\right\rangle=-i e C_{4}^{\prime}\left(\boldsymbol{\sigma}_{1}+\boldsymbol{\sigma}_{2}\right) \times\left[e_{1}\left(\mathbf{p}_{2}-\mathbf{p}_{2}^{\prime}\right)+e_{2}\left(\mathbf{p}_{1}-\mathbf{p}_{1}^{\prime}\right)\right] \cdot \frac{\hat{\mathbf{e}}_{\mathbf{q} \lambda}}{\sqrt{2 \omega_{q}}}, \\
& \left\langle H_{\mathrm{CT} \gamma, 5}\right\rangle=-i e C_{5}^{\prime}\left[e_{1} \boldsymbol{\sigma}_{1} \times\left(\mathbf{p}_{1}-\mathbf{p}_{1}^{\prime}\right)+e_{2} \boldsymbol{\sigma}_{2} \times\left(\mathbf{p}_{2}-\mathbf{p}_{2}^{\prime}\right)\right] \cdot \frac{\hat{\mathbf{e}}_{\mathbf{q} \lambda}}{\sqrt{2 \omega_{q}}}, \\
& \left\langle H_{\mathrm{CT} \gamma, 6}\right\rangle=\quad i e C_{6}^{\prime}\left[e_{1} \boldsymbol{\sigma}_{2} \times\left(\mathbf{p}_{1}-\mathbf{p}_{1}^{\prime}\right)+e_{2} \boldsymbol{\sigma}_{1} \times\left(\mathbf{p}_{2}-\mathbf{p}_{2}^{\prime}\right)\right] \cdot \frac{\hat{\mathbf{e}}_{\mathbf{q} \lambda}}{\sqrt{2 \omega_{q}}}, \\
& \left\langle H_{\mathrm{CT} \gamma, 7}\right\rangle=2 e\left[C_{7}^{\prime}\left[e_{1}\left(\mathbf{p}_{2}+\mathbf{p}_{2}^{\prime}\right) \cdot \boldsymbol{\sigma}_{2} \boldsymbol{\sigma}_{1}+e_{2}\left(\mathbf{p}_{1}+\mathbf{p}_{1}^{\prime}\right) \cdot \boldsymbol{\sigma}_{1} \boldsymbol{\sigma}_{2}\right]\right. \\
& +C_{8}^{\prime}\left[e_{1}\left(\mathbf{p}_{2}+\mathbf{p}_{2}^{\prime}\right) \cdot \boldsymbol{\sigma}_{1} \boldsymbol{\sigma}_{2}+e_{2}\left(\mathbf{p}_{1}+\mathbf{p}_{1}^{\prime}\right) \cdot \boldsymbol{\sigma}_{2} \boldsymbol{\sigma}_{1}\right] \\
& \left.+C_{9}^{\prime} \boldsymbol{\sigma}_{1} \cdot \boldsymbol{\sigma}_{2}\left[e_{1}\left(\mathbf{p}_{2}+\mathbf{p}_{2}^{\prime}\right)+e_{2}\left(\mathbf{p}_{1}+\mathbf{p}_{1}^{\prime}\right)\right]\right] \cdot \frac{\hat{\mathbf{e}}_{\mathbf{q} \lambda}}{\sqrt{2 \omega_{q}}}, \\
& \left\langle H_{\mathrm{CT} \gamma, 8}\right\rangle=-e\left[C_{10}^{\prime}\left[e_{1}\left(\mathbf{p}_{2}+\mathbf{p}_{2}^{\prime}\right) \cdot \boldsymbol{\sigma}_{2} \boldsymbol{\sigma}_{1}+e_{2}\left(\mathbf{p}_{1}+\mathbf{p}_{1}^{\prime}\right) \cdot \boldsymbol{\sigma}_{1} \boldsymbol{\sigma}_{2}\right]\right. \\
& +C_{11}^{\prime}\left[e_{1}\left(\mathbf{p}_{2}+\mathbf{p}_{2}^{\prime}\right) \cdot \boldsymbol{\sigma}_{1} \boldsymbol{\sigma}_{2}+e_{2}\left(\mathbf{p}_{1}+\mathbf{p}_{1}^{\prime}\right) \cdot \boldsymbol{\sigma}_{2} \boldsymbol{\sigma}_{1}\right] \\
& \left.+C_{12}^{\prime} \boldsymbol{\sigma}_{1} \cdot \boldsymbol{\sigma}_{2}\left[e_{1}\left(\mathbf{p}_{2}+\mathbf{p}_{2}^{\prime}\right)+e_{2}\left(\mathbf{p}_{1}+\mathbf{p}_{1}^{\prime}\right)\right]\right] \cdot \frac{\hat{\mathbf{e}}_{\mathbf{q} \lambda}}{\sqrt{2 \omega_{q}}}, \\
& \left\langle H_{\mathrm{CT} \gamma, 9}\right\rangle=-e\left[C _ { 1 3 } ^ { \prime } \left[e_{1}\left(\mathbf{p}_{1}+\mathbf{p}_{1}^{\prime}\right) \cdot \boldsymbol{\sigma}_{1} \boldsymbol{\sigma}_{2}+e_{1}\left(\mathbf{p}_{1}+\mathbf{p}_{1}^{\prime}\right) \cdot \boldsymbol{\sigma}_{2} \boldsymbol{\sigma}_{1}\right.\right. \\
& \left.+e_{2}\left(\mathbf{p}_{2}+\mathbf{p}_{2}^{\prime}\right) \cdot \boldsymbol{\sigma}_{1} \boldsymbol{\sigma}_{2}+e_{2}\left(\mathbf{p}_{2}+\mathbf{p}_{2}^{\prime}\right) \cdot \boldsymbol{\sigma}_{2} \boldsymbol{\sigma}_{1}\right]
\end{aligned}
$$




$$
\left.+2 C_{14}^{\prime} \boldsymbol{\sigma}_{1} \cdot \boldsymbol{\sigma}_{2}\left[e_{1}\left(\mathbf{p}_{1}+\mathbf{p}_{1}^{\prime}\right)+e_{2}\left(\mathbf{p}_{2}+\mathbf{p}_{2}^{\prime}\right)\right]\right] \cdot \frac{\hat{\mathbf{e}}_{\mathbf{q} \lambda}}{\sqrt{2 \omega_{q}}}
$$

[1] S. Weinberg, Phys. Lett. B251, 288 (1990); Nucl. Phys. B363, 3 (1991); Phys. Lett. B295, 114 (1992).

[2] U. van Kolck, Phys. Rev. C 49, 2932 (1994); C. Ordónez, L. Ray, and U. van Kolck, Phys. Rev. C 53, 2086 (1996).

[3] E. Epelbaum, W. Glöckle, and U.-G. Meissner, Nucl. Phys. A637, 107 (1998); Nucl. Phys. A747, 362 (2005).

[4] T.-S. Park et al., Phys. Rev. C 67, 055206 (2003).

[5] S.F. Mughabghab, M. Divadeenam, and N.E. Holden, Neutron Cross Sections from Neutron Resonance Parameters and Thermal Cross Sections (Academic Press, London, 1981).

[6] E.T. Jurney, P.J. Bendt, and J.C. Browne, Phys. Rev. C 25, 2810 (1982).

[7] F.L.H. Wolfs, S.J. Freedman, J.E. Nelson, M.S. Dewey, and G.L. Greene, Phys. Rev. Lett. 63, 2721 (1989); R. Wervelman, K. Abrahams, H. Postma, J.G.L. Booten, and A.G.M. Van Hees, Nucl. Phys. A526, 265 (1991).

[8] J. Carlson and R. Schiavilla, Rev. Mod. Phys. 70, 743 (1998).

[9] L.E. Marcucci, M. Viviani, R. Schiavilla, A. Kievsky, and S. Rosati, Phys. Rev. C 72, 014001 (2005).

[10] A. Kievsky, S. Rosati, M. Viviani, L.E. Marcucci, and L. Girlanda, J. Phys. G 35, 063101 (2008).

[11] D.O. Riska, Phys. Scr. 31, 471(1985); R. Schiavilla, V.R. Pandharipande, and D.O. Riska, Phys. Rev. C 40, 2294 (1989); J. Carlson, D.O. Riska, R. Schiavilla, and R.B. Wiringa, Phys. Rev. C 42, 830 (1990).

[12] A. Buchmann, W. Leidemann, and H. Arenhövel, Nucl. Phys. A443, 726 (1985); H. Arenhövel, F. Ritz, and T. Wilbois, Phys. Rev. C 61, 034002 (2000).

[13] K.-M. Schmitt, P. Wilhelm, H. Arenhövel, Few-Body Syst. 10, 105 (1991); R. Schiavilla, Phys. Rev. C 72, 034001 (2005).

[14] L.E. Marcucci, M. Pervin, S.C. Pieper, R. Schiavilla, and R.B. Wiringa, submitted to Phys. Rev. C, arXiv:0810.0547.

[15] R. Schiavilla, R.B. Wiringa, J. Carlson, and V.R. Pandharipande, Phys. Rev. C 45, 2628 (1992).

[16] T.-S. Park, D.-P. Min, and M. Rho, Nucl. Phys. A596, 515 (1996).

[17] Y.-H. Song, R. Lazauskas, T.-S. Park, and D.-P. Min, Phys. Lett. B656, 174 (2007).

[18] T.-S. Park, K. Kubodera, D.-P. Min, and M. Rho, Phys. Lett. B472, 232 (2000).

[19] J.L. Goity, S. Pastore, and R. Schiavilla, in preparation.

[20] R.B. Wiringa, V.G.J. Stoks, and R. Schiavilla, Phys. Rev. C 51, 38 (1995).

[21] R. Machleidt, Phys. Rev. C 63, 024001 (2001).

[22] B.S. Pudliner et al., Phys. Rev. C 56, 1720 (1997).

[23] M. Viviani et al.. Phys. Rev. Lett. 99, 112002 (2007).

[24] J.D. Bjorken and S.D. Drell, Relativistic Quantum Mechanics (McGraw-Hill, Inc., 1964).

[25] D.O. Riska and G.E. Brown, Phys. Lett. B38, 193 (1972).

[26] V.G.J. Stoks, R.A.M. Klomp, C.P.F. Terheggen, and J.J. deSwart, Phys. Rev. C 49, 2950 (1994). 
[27] V. Burkert, private communication.

[28] R. Schiavilla, J. Carlson, and M. Paris, Phys. Rev. C 70, 044007 (2004).

[29] M. Viviani, R. Schiavilla, and A. Kievsky, Phys. Rev. C 54, 534 (1996).

[30] M.W. Konijnenberg et al., Phys. Lett. B205, 215 (1988).

[31] H. Sadeghi, S. Bayegan, and H. Griesshammer, Phys. Lett. B643, 263 (2006); H. Sadeghi, Phys. Rev. C 75, 044002 (2007). 